\title{
COMPUTATION OF RAPIDLY VARIED, UNSTEADY, FREE-SURFACE FLOW
}

\author{
By David R. Basco
}

Water-Resources Investigations Report 83-4284

\author{
Reston, Virginia
}

1987 
DEPARTMENT OF THE INTERIOR

DONALD PAUL HODEL, Secretary

U.S. GEOLOGICAL SURVEY

Dallas L. Peck, Director

For additional information write to:

Chief, Branch of Regional Research, NR Water Resources Division U.S. Geological Survey 432 National Center 12201 Sunrise Valley Dr. Reston, Virginia 22092
Copies of this report can be purchased from:

U.S. Geological survey Books and Open-File Reports Section Federal Center Box 25425

Denver, Colorado 80225

Use of trade names and trademarkes in this publication is for descriptive purposes only and does not constitute endorsement by the U.S. Geological survey. 
Abstract Introduction . . . . . . . . . . . . . . . . . . 2

Literature Review . . . . . . . . . . . . . . . 4

Waves of Permanent and Nonpermanent Form. . . . . . . . 4

Modern Derivation Methods ............... . 5

Asymptotic Expansion Method. . . . . . . . . 6

Horizontal Bottom ............... 7

Variable Water Depth. . . . . . . . . 8

Variation Principles ... . . . . . . . . 9

Horizontal Bottom . . . . . . . . . . 11

Small Amplitude Theory . . . . . . . . 11

Finite Amplitude Theory. . . . . . . . 12

Variable Water Depth. . . . . . . . . 13

Conservation Methods ................. 14

Summary of Equations . . . . . . . . . . . . 16

Arbitrary Cross-Sections. . . . . . . . . . . 18

Theory . . . . . . . . . . . . . . . . . 19

Conservation Form Following Venezian. . . . . . . . 19

Conservation Integrals .. . . . . . . . . 20

Continuity Integral ............ 20

Horizontal Momentum Integral. ......... 21

Energy Integral ............... 21

Vorticity Integral. .............. 21

Long Wave Propagation. . . . . . . . . . . 22 
Quasi-Iong Wave Propagation. Page

Eulerian Form . . . . . . . . . . . . 24

Conservation Form .............. 25

Nondimensional Equations. . . . . . . . . . . 26

Mass Conservation. . . . . . . . . . . . 27

Momentum Conservation. . . . . . . . . . . . 28

Bottom Variation Scaling ............. 29

Bed Gradient Terms. . .............. 29

Bottom Curvature Terms. . . . . . . . . 29

Summary, Nondimensional Equations. . . . . . . . 30

Boundary conditions . . . . . . . . . . . . . 30

Practical consideration . . . . . . . . . . . 31

Perturbation Analysis. . . . . . . . . . . . . . . . 32

The Perturbed Equations . . . . . . . . . . . 32

Long Wave Equations. . . . . . . . . . . . 32

Boussinesq-Type Equations. . . . . . . . . . . 34

simplified Flow Cases ............... 36

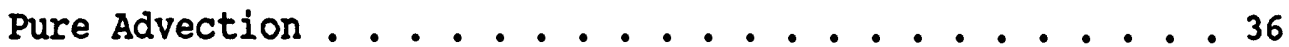

Lagrange Celerity. . . . . . . . . . . 36

Long Waves with Friction .. . . . . . . . 37

Diffusion and Kinematic Wave Models. . . . . . . . 37

Linearized Boussinesq Equation . . . . . . . 38

Classic Boussinesq Equation. . . . . . . . . . 38

Boussinesq Theory on Plane Slope with Friction. . . . . . 39

Summary of Cases Tested. . . . . . . . . . . . 42

Case 4 Linearized Boussinesq. . . . . . . . 42 
Case 7 Full, Long Wave Theory ........... 42

Case 9 Boussinesq Equations ............ 46

Case 10, 11, 12, and 13, The $b_{2}$ Term. . . . . . . 46

Case 14 Boussinesq Equation with Friction . . . . 46

Cases 15 and 16, The Slope-Boussinesq Term $\left(b_{s}\right) . . .47$

Conclusions from Perturbation Analysis. . . . . . . . . 47

Continuum Equations for Numerical Integration . . . . . . 48

Numerical Integration Methods. . . . . . . . . . . . 49

Method of Characteristics .............. 49

Finite-Difference Methods ............... 49

Present Methods. .................. 49

Future Methods................. 56

Filter Scheme Methods . . . . . . . . . . . 57

Conclusions and suggestion for Further study . . . . . . . . . 58

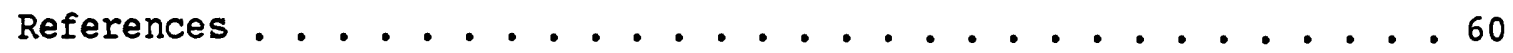

Appendix . . . . . . . . . . . . . . . . . . 65 


\section{FIGURES}

Page

Figure 1. Generalized diagram showing definition sketch. . . . . 8

2-7 Graphs showing:

2. Range of flow conditions tested. .......... 41

3. Range of applicability of linearized

Boussinesq equations ............... 44

4. Example dimensionless wave celerity, $y_{+}$as function

of wave number for $F_{0}=0.1$, Boussinesq equations. . 45

5. Amplitude variation of a periodic wave during shoaling.

(from Madsen, Mei and Savage, 1970). . . . . . 5 50

6. Values of (a) $a_{2} / \mathrm{h}$ and (b) $a_{3} / \mathrm{h}$ as a function of incident angle for Perroud's study (dotted lines) and numerical study (solid lines) of Hebenst4reit and Reid (1978). . 52

7. Comparison of numerical computations of shoaling waves obtained by Abbott et al. $(1978 \mathrm{a}, \mathrm{b})$ in one dimensional as compared with experimental results of Madsen and Mei (1969) . . . . . . . . . . 54

8. The implicit Preissman or "Box" scheme for (a) two-time levels or (b) three-time levels as needed for the Boussinesq equations 


\section{TABLES}

Page

Table 1. Summary of Boussinesq-Type equations of $\mathrm{m} \quad$. . . . 17

2. Non-dimensional derivative terms in fundamental

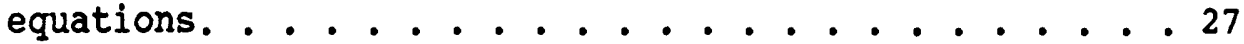

3. Celerity Tests ................ 40

4. Example results of test No. $2\left(s_{0}=0.001, h_{0}=12\right.$,

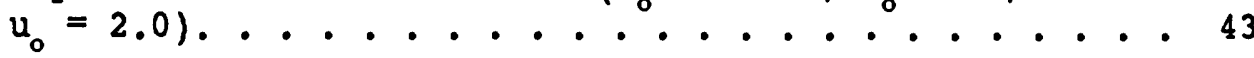


FACTORS FOR CONVERTING INCH-POUND UNITS TO INTERNATIONAL SYSTEM OF UNITS (SI)

For the convenience of readers who may want to use International system of Units (SI), the data may be converted by using the following factors:

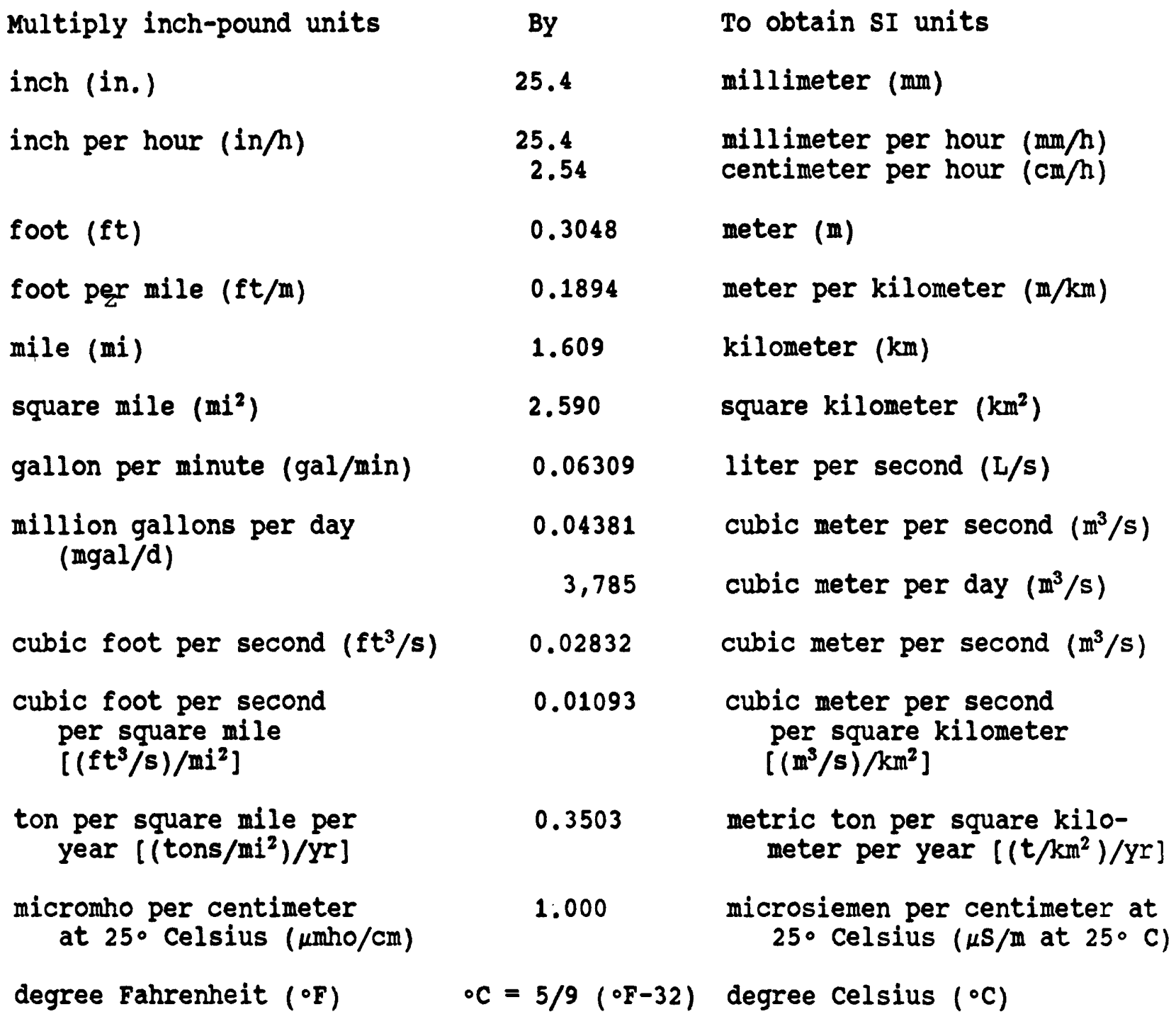

National Geodetic Vertical Datum of 1929 (NGVD of 1929): A geodetic datum derived from a general adjustment of the first-order level nets of both the United states and Canada, formerly called mean sea level. NGVD of 1929 is referred to as sea level in this report. 


\section{NOMENCLATURE}

a

$$
a_{2}, a_{3}
$$

A

$b_{1}$

$\mathrm{b}_{2}$

$b_{8}$

B

C

$C_{B}$

C

LB

$c_{t}$

$c_{1}$

$c_{2}$

d

D

e, $\exp$

$f, f_{0}, f_{1}, f_{2}$, etc.

f.

$\mathbf{F}$

$F_{r}, F_{0}$ coefficient ( 0 or 1 ) concerned with convective acceleration term in motion eqn.

wave amplitudes from study by Perroud (1957).

a coefficient in Mass and Vastano (1978).

coefficient ( 0 or 1 ) concerned with primary

Boussinesq term.

coefficient ( 0 or 1 ) concerned with secondary Boussinesq term

coefficient ( 0 or 1 ) concerned with slope Boussinesq term.

a coefficient; the key coefficient in Venezian (1976).

the celerity of shallow-water wave propagation.

the celerity of full Boussinesq equations.

the celerity of linearized Boussinesq equations.

coefficient ( 0 or 1 ) concerned with time derivative in continunity.

coefficient ( 0 or 1 ) concerned with hu term in continuity equation (prism-storage term).

coefficient ( 0 or 1 ) concerned with uh term in continuity equation (wedge-storage term).

the local still water depth above channel bottom.

the local still water depth above reference datum; also the density (mass, momentum, energy, vorticity) per unit width.

the exponential.

a dummy variable, higher order terms of $f$.

value of $f$ on free water surface.

the flux (mass, momentum, energy, vorticity) per unit width.

the Froude number; uniform flow Froude number. 
$g, g *$

$h, h *$ or $\underline{h}$

$h_{0} ; h^{\prime}$

H

i

i

$I, J, K, M, N$,

$P, Q, R$

$j ; j j$

j

k

$\mathbf{k}$

$\mathbf{K}$

$\ell$

L

m

$n ; n n$

$\mathrm{p}$

$\mathrm{p}_{8}$

$\mathbf{P}_{\mathrm{D}}$

q

$\mathbf{R}$

s。

$t ; \Delta t$ the acceleration of gravity; dimensionless.

the water depth; dimensionless water depth.

uniform water depth; water depth fluctuation.

the total wave height.

imaginary number index.

the unit normal vector in the $\mathrm{x}$-direction.

coefficients in derivation by Mass and Vastano (1978).

grid index in $x$-direction; final grid value.

the unit normal vector in the $y$-direction.

an index counter $(0,1,2,3, \ldots)$; also the coefficient

$(0$ or 1 ) associated with the friction-bed slope term in

the motion equation.

the wave number, $2 \pi / \mathrm{L}$.

the unit normal vector in the $z$-direction.

the diffusion or dispersion codfficient.

the coefficient ( 0 or 1 ) concerned with the local

acceleration term in the motion equation.

the wave length; the Lagrangian.

conservation of mass in Lagrangian multiplier method.

the time index; the final index value.

pressure; also the coefficient ( 0 or 1 ) concerned with the pressure gradient term in motion equation.

pressure on water surface.

the Peclet number.

volumetric flowrate per unit width.

a coefficient.

uniform flow bed slope.

time; time increment. 
T

$u, u *$ or $\underline{u}$

$u_{0} ; u^{\prime}$

$\overline{\mathrm{u}}$

U

v

$w_{1} w_{0}, w_{1}, w_{2}$, etc.

$w_{0}$

$x ; \Delta x$

$\dot{\mathbf{x}}$

$y$

$\mathbf{z}$

Greek symbols

$\boldsymbol{\alpha}$

$\alpha_{0}$

B

$\gamma$

$\gamma_{0}, \gamma_{a}$

$\gamma_{+}\left(\gamma_{1}\right)$

$\gamma_{-}\left(\gamma_{2}\right)$

$\gamma_{B}$ kinetic energy per unit mass and depth integrated; also the wave period.

the $x$-direction velocity, dimensionless water velocity. uniform flow velocity or depth averaged velocity; velocity flucuation. the time mean water velocity. the Ursell parameter.

the potential energy per unit mass and depth integrated. the vertical velocity and higher order terms of $w$. the integration constant. space coordinate direction; increment in $\mathrm{x}$. the Lagrange celerity $u \pm \sqrt{g d}$. space coordinate direction. vertical space coordinate direction.

the dimensionless wave number, $\epsilon$ (or $\Delta x$ ); also used by Haugel(1980) to represent the total (substantive) second derivative of $h$.

the dimensionless wave number, $h_{0}$.

the local bottom slope; also used by Haugel (1980) to represent the total second derivative of $\xi$ and; $a$ dimensionless term in perturbation analysis.

the courant number (i.e. the dimensionless wave celerity).

ratios (dimensionless) used in perturbation study. positive direction, dimensionless wave celerity. negative direction, dimensionless wave celerity. dimensionless wave celerity for full Boussinesq equations. 


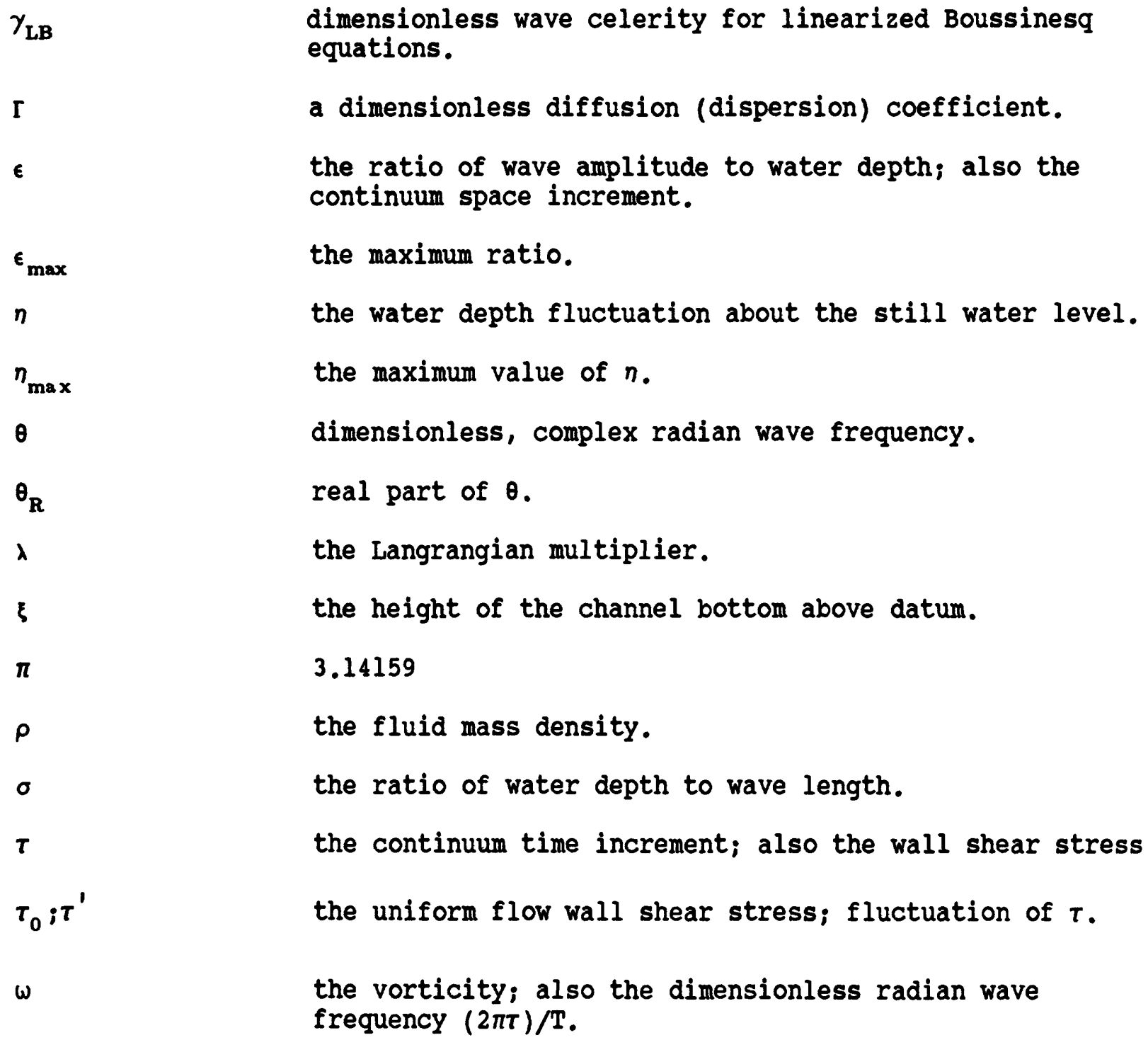
the vorticity; also the dimensionless radian wave frequency $(2 \pi T) / T$.

dimensionless wave celerity for linearized Boussinesq equations.

a dimensionless diffusion (dispersion) coefficient. the ratio of wave amplitude to water depth; also the continuum space increment.

the maximum ratio.

the water depth fluctuation about the still water level. the maximum value of $n$. dimensionless, complex radian wave frequency. real part of $\theta$. the Langrangian multiplier. the height of the channel bottom above datum. 3.14159

the fluid mass density. the ratio of water depth to wave length. the continuum time increment; also the wall shear stress the uniform flow wall shear stress; fluctuation of $\tau$.

Abbreviations

H.O.T.

higher order terms

$\mathrm{KdV}$ Korteweg - de Vries equations

PDE partial differential equations

SWL still water level

T.E. truncation errors 
ㄴ.

*

\section{Special symbols}

$\approx$

$<$

3

$\boldsymbol{\nabla}$

$0(1)$

$\frac{\partial}{\partial x}, \frac{\partial}{\partial t}$ partial differentiation

overbar means time mean

underbar means dimensionless

dimensionless quantity

approximately equal

much less than

greater than or equal to

vector operator $\mathbf{i} \frac{\partial}{\partial \mathrm{x}}+\mathbf{j} \frac{\partial}{\partial \mathrm{y}}+\mathbf{k}(0)$

order-of-magnitude of ( )

partial differentiation 
COMPUTATION OF RAPIDLY VARIED,

UNSTEADY, FREE-SURFACE FLOW

By David R. Basco

\section{ABSTRACT}

The correct physical simulation of unsteady, free surface flows with large surface gradients remains an important research area of computational hydraulics. Examples are spiked river hydrographs in shallow streams from natural runoff and solitary wave type disturbances from rapid reservoir releases, dam breaks and mud slides into rivers due to volcanic eruption. For these flows, the long wave equations of motion (St. Venant) which assume a hydrostatic pressure distribution over the depth are no longer valid. The purpose of this report is to present an introductory review of both the Boussinesq-type, differential equations to describe these flows and the methods for their numerical integration.

Three independent methods to derive the appropriate equations were reviewed. For small amplitude disturbances of a specified scale on horizontal slopes they all gave identical results. On variable slopes and for larger scale (finite-amplitude) disturbances, all three methods gave different results for the additional, higher order terms. Through continuity, they may still be equivalent to a prescribed order of accuracy but this was not confirmed in this study. Further research is needed to determine the importance of these higher-order terms for riverine applications.

A perturbation analysis was made to quantify the significant reduction in wave celerity due to the added Boussinesq term over that for the non-linear, long wave equations of St. Venant. It was also determined that for Froude numbers greater than 0.05 (based upon uniform flow), the full, nonlinear Boussinesq equations (from the small amplitude theory) are required to compute wave celerity with 5 percent accuracy.

Great care must be exercised to eliminate truncation errors from finite-difference schemes of the Boussinesq equations. Truncation errors can cause numerical frequency dispersion to swamp the true physical dispersion of the added Boussinesq term(s). The three-level scheme (time) reviewed may not be the best analog because diverging solutions at alternating time steps appear.

It is recommended that the next phase in the effort concentrate on firmly establishing the practical hydraulics cases when the more general Boussinesq equation must be employed. And, to run some typical numerical examples to demonstrate the order of differences in solution with those obtained from the long wave equations of st. Venant. 


\section{INTRODUCTION}

Many unsteady flows in hydraulics occur with relatively large gradients in free surface profile. The assumption of a hydrostatic pressure distribution with depth is no longer valid. These are rapidly-varied unsteady flows (RVF) of classical hydraulics and also encompass short wave propagation of coastal hydraulics. Numerical methods are needed to readily calculate rapidly-varied unsteady flows together with computations of long wave nearly horizontal, gradually varied flows (GVF). Transition between hydrostatic and nonhydrostatic flow regimes should be a fundamental part of the equations of motion employed and automatically a part of the solution algorithms generated. Artificial separation between RVF and GVF regimes and the piecing together of solutions is unrealistic for unsteady flow computations. The location of the RVF region varies continuously and becomes part of the desired solution.

Spiked river hydrographs occurring from natural runoff in steep gorges, canyons and channels are an example of rapidly-varied unsteady free surface flows. They also result from rapid reservoir releases, dam breaks and mud slides into rivers due to volcanic eruptions. Flows over hydraulic structures such as spillways, chutes, control and measurement devices (weirs, flumes, gates), energy dissipators, buckets, and near sudden slope changes all create rapidly-varied flows. In these cases the bottom curvature effects are also important. When the free surface becomes too steep and unstable, roll waves, hydraulic jumps, bores, breaking waves and other discontinuities propagate in the flow. Automatic shock propagation is also desirable in the solution.

The purpose of this report is to present an introductory review of the state of knowledge and numerical computation techniques to solve such flow problems. First a review of the literature is made and various methods employed to derive the appropriate equations of motion are presented. These are then summarized in a table and discussed in detail since the appropriate equations to use is still a subject of active research. Further insight is given in the next section where the equations are rederived following the methods of Venezian (1974)* and a nondimensional analysis of the equations is displayed. Boundary conditions are also reviewed in this section. Perturbation analysis efforts are presented in the following section. such methods permit determination of the propagation celerity and response matrix for various cases when certain terms are included or omitted in the equations. It is then used as a tool to determine the relative importance of these terms in the equations employed. The hydrodynamic stability of the equations for roll wave formation can also be ascertained. The section concludes with the selection of the appropriated equations for numerical integration. Subsequently, in another section reviews are given for various numerical integration methods including the finite-difference methods and their requirements for stability and accuracy when applied to rapidly-varied flows. Recommendations for appropriate integration schemes are presented. Conclusions and recommendations are summarized in the last section of this report.

*Complete reference information is found in the References cited section at the end of this report. 
The continued encouragement of $\mathrm{Dr}$. Chintu Lai of the USGS for conducting this research through his computational hydraulics projects is gratefully acknowledged. The writer also appreciates the review and comments by Dr. Guilio Venezian, Texas A\&M University and Mr. Robert E. Jensen, USAE, Waterways Experiment station.

It is hoped that this is the first in many important steps necessary to simulate rapidly-varied, free-surface flows. 


\section{LITERATURE REVIEW}

The theory that incorporates vertical accelerations, to a limited extent, in approximations to the horizontal motion equation is called Boussinesq theory (Boussinesq, 1872). Many forms of the equations attributable to Boussinesq are found in the literature. Variations are due to the order of accuracy of terms retained and methods of derivation. In fact, the basic form of the Boussinesq equations found in the recent literature (e.g., Whitham, 1974 ) never appears in Boussinesq's work (Vastano and Mungall, 1976). Simplification of the equations by limiting wave propagation to only one direction (no reflections) gives the equations of Korteweg and de Vries (1895) or KdV equations. These are of less generality and will be omitted in what follows. However, exact solutions of the KdV equations are termed first-order cnoidal waves. The solitary wave is a limiting case of a cnoidal wave. Thus these classical wave theories have their roots in Boussinesq theory, or vice versa.

The basic Boussinesq equations in one dimension for uniiform cross-section, on a horizontal bottom neglecting bed and surface stresses can be written in Eulerian form

Mass

$$
h_{t}+(d) u_{x}+u h_{x}=0
$$

Motion

$$
u_{t}+u u_{x}+g h_{x}=\frac{1}{3}\left(d^{2}\right) u_{x \times t}
$$

where $h=n+d$ with $d$ the uniform flow or still water depth and $n$ the instantaneous water surface variation. The velocity $u$ is a depth-averaged instantaneous value. Subscripts indicate partial differentiation in either independent variable $x$ - in space or $t$ - in time. The equation of motion is identical to the long wave equation of saint-Venant except for the mixed derivative Boussinesq term on the right-hand side (RHS) of Equation (2). It is instructive to briefly review various forms which omit certain terms in Equations (1) and (2).

\section{Waves of Permanent and Nonpermanent Form}

If the convective acceleration and Boussinesq terms are neglected, a wave of permanent form moving with speed (celerity) $c=(g d)^{1 / 2}$ results. Without only the Boussinesq term, each part of the solution travels at a local speed $\dot{x}=u f(g h)^{1 / 2}$ so that the high parts tend to overtake the low sections with time. The wave travels with nonpermanent form, continually steepens and eventually breaks. This tendency is termed amplitude dispersion. If, however, the convective acceleration term and $h_{x}$ term in the mass equation are omitted, a linearized version of the Boussinesq equations results. Vertical accelerations resulting from streamline curvature effects result in the celerity of the form (See, e.g., Whitham, 1974, p. 462) 


$$
c_{L B}=(g d)^{1 / 2} /\left[1+\left(4 \pi^{2} d^{2} / 3 L^{2}\right)\right]^{1 / 2}
$$

where $L$ is the wave length of any wave component in the solution. Each wave (Fourier) component of a long wave disturbance now travels at its own speed depending on its length. This tendency is termed frequency (or wave-length) dispersion. The full equations with all terms maintain a balance between amplitude and frequency dispersion only for the limiting case of the solitary wave. They also simulate a progressive wave of permanent form (first order, cnoidal) without excessive dispersion if the wave does not propagate indefinitely (Peregrine, 1972).

Ursell (1953) devised the following criterion, the Ursell number, $U$, for applying the Boussinesq equation or special cases with terms omitted.

When

$$
U\left\{\begin{array}{cl}
\gg 1 & \text { Nonlinear, longwave equations } \\
0(1) & \text { Boussinesq equations } \\
\ll 1 & \text { Linear wave equations }
\end{array}\right.
$$

where

$$
\begin{aligned}
U & =(\text { wave amplitude/water depth })^{3} /(\text { wave steepness })^{2} \\
& =\left(n_{\max } / d\right)^{3} /\left(n_{\max } / L\right)^{2}
\end{aligned}
$$

or

$$
U=\left(n_{\max } L^{2}\right) / d^{3}
$$

and the symbol $O(1)$ means the value is on the order of unity. The region where $U$ is comparable to unity is cited as applicable to cnoidal wave theory (e.g., Peregrine, 1967) which has been shown to match experimental water surface and velocity profiles (Wiegel, 1964) for finite amplitude, progressive waves in shallow water. Therefore, the Boussinesq equations can be considered the more general set with the Saint Venant equations a special case for long waves such as tides and sluggish river hydrographs.

For sloping and variable bottom profiles, the equation of motion becomes much more complicated. For adverse slopes (coastal beaches) no waves of permanent form can exist. The wave shoals, peaks and eventually breaks when $\mathrm{H} / \mathrm{d} \approx 0.8-1.0$ where $\mathrm{H}$ is the trough to peak wave height. The applicability of the Boussinesq equations up to the breaking limit on beaches is an active research area. On positive slopes (rivers, estuaries) permanent form waves are possible but only for short reaches. No known research has been conducted on this topic.

\section{Modern Derivation Methods}

The Boussinesq theory can be considered applicable for finite-amplitude quasi-long waves propagating in shallow water where the interplay between nonlinearity (convective acceleration term), dispersiveness (Boussinesq term) 
and bottom curvature or slope (additional terms) are critically intertwined. Early rederivations by Keulegan and Patterson (1940) and Ursell (1953) were restricted to wave propagation in only one direction which greatly limited their usefulness. More recently, three different derivational methods have emerged that do permit arbitrary disturbance propagation.

\section{Asymptotic Expansion Method}

Peregrine (1966, 1967) and Mei and Léméhauté (1966) were the first to employ expansion procedures to derive Boussinesq equations for water of variable depth. Mei and LeméHaute used the velocity at the bottom as reference and the governing equations were put in characteristic form, making them less general. Additional work with these equations can be found in Madsen and Mei (1969), and Madsen, Mei and Savage (1970) for solitary waves and solitons, respectively.

Peregrine (1967) began with Euler's equations of motion for an inviscid fluid. Two scaling parameters are then introduced. The importance of the nonlinear (convection) term in amplitude dispersion is given by $\epsilon$, where

$$
\begin{aligned}
\epsilon & =\text { wave amplitude/water depth } \\
& =\eta_{\max } / \mathrm{d}
\end{aligned}
$$

The Boussinesq term gives frequency dispersion and is related to the parameter $\sigma^{2}$, where $\sigma$ is the relative depth

$$
\begin{aligned}
\sigma & =\text { water depth/wave lenth } \\
& =d / L
\end{aligned}
$$

Both $\epsilon$ and $\sigma^{2}$ are relatively small, i.e. much less than unity. In terms of the Ursell number, using equations (6) and (7) in (5) gives

$$
U=\frac{\epsilon}{\sigma^{2}}
$$

From equation (4) it is apparent that the Boussinesq equations are applicable when amplitude dispersion given by $\epsilon$ and frequency dispersion given by $\sigma^{2}$ are equally important, i.e.

$$
\epsilon \approx \sigma^{2} \quad \text { (for Boussinesq equations) }
$$

Following Keller (1948), Peregrine expanded all variables as follows. The dependent variables, $n, p$, and $u$ were expanded in terms of the nonlinearity parameter, $\epsilon$ as

$$
f=f_{0}+\epsilon f_{1}+\epsilon^{2} f_{2}+\ldots .
$$

with $f$ a dummy variable standing for $n, p$, or $u$. The vertical velocity, $w$ was expanded in terms of the frequency dispersion parameter, $\sigma$ and also $\epsilon$ as 


$$
w=\sigma\left(w_{0}+\epsilon W_{1}+\epsilon^{2} W_{2}+\ldots . \cdot\right)
$$

The independent variables were scaled by $\sigma$, for example

$$
\frac{\partial}{\partial x}=\sigma\left(\frac{\partial}{\partial x_{1}}\right) ; \text { etc. }
$$

Using the still water depth and $\sqrt{g d}$ as reference length and velocity, respectively, all equations were put in dimensionless form. All expanded variables and their derivatives were assumed to be of order $O(1)$ so that when the expansions were made, the order-of-magnitude of each term in the equations appeared explicitly. The zero-order solution gave the equation of statics and the first-order equations were the linearized, long wave equations.

\section{Horizontal bottom}

The second-order equations have first-order effects which to be included, the first-order variables incorporating the second-order terms are employed. The results are normally expressed in terms of the mean, velocity although others have used the velocity at the bed (Mei and Le'MeHaute , 1966; Dressler, 1978). In dimensionless terms, the Boussinesq equations for horizontal bottom derived by the expansion method became

Mass: $\quad \underline{h}_{t}+\bar{u}_{x}+\epsilon \bar{u}_{-x}+O\left(\epsilon \sigma^{2}, \epsilon^{2}, \ldots ..\right)=0$

Motion: $\quad \bar{u}_{t}+\epsilon \bar{u} \bar{u}_{x}+\underline{h}_{x}-\frac{1}{3} \sigma^{2} \bar{u}_{x o x t}+o\left(\epsilon \sigma^{2}, \epsilon^{2}, \ldots ..\right)=0$

Note that all terms are dimensionless ( $g=1$; the underbar) the overbar signifies mean velocity, and $\eta$ could have replaced $\underline{h}$ since $d=1, d_{x}=0$. clearly, both the convective acceleration term and Boussines $\bar{q}$ term are equally important when $\epsilon=\sigma^{2}$. All additional terms of order $\epsilon \sigma^{2}, \epsilon^{2}$, etc. are lumped together because of higher order. Equations (13) and (14) are identical to equations (1) and (2) when returning to dimensional variables. For further discussion with full derivations see Peregrine (1972) and Whitman (1974). study of the derivation shows the additional Boussinesq term results from both the horizontal velocity and pressure distribution being influenced by the vertical velocity.

Equation (13) for mass conservation also reveals the important fact that to the lowest order accuracy (i.e. linear theory)

$$
\bar{u}_{x}=-\underline{h}_{t}+O\left(\epsilon, \sigma^{2}\right)
$$

or

$$
\bar{u}_{x x t}=-\underline{h}_{x t t}+O\left(\epsilon, \sigma^{2}\right)
$$

Now as discussed in great detail by Long (1964), one can always substitute the lowest order approximation into the Boussinesg term which is the next higher order. Putting Equation (16) into (14) gives 


$$
\bar{u}_{t}+\epsilon \bar{u} \bar{u} \bar{x}_{x}+\underline{h}_{x}+\frac{1}{3} \sigma^{2} \underline{h}_{x t t}+O\left(\epsilon \sigma^{2}, \epsilon^{2}\right)=0
$$

which is an equivalent form. It is thus seen that where the free surface varies most rapidly, the Boussinesg term will have the most influence on the solution. This is the regime of rapidly-varied unsteady flow.

Variable water depth

Consider the difinition sketch

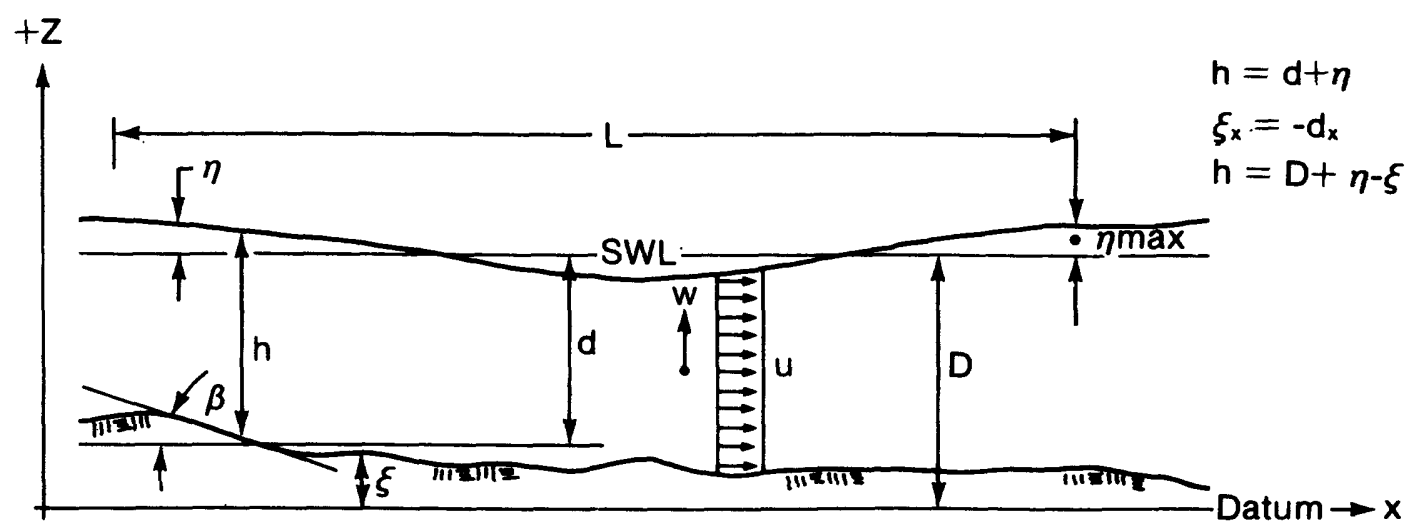

Figure 1. Generalized diagram showing definition sketch.

of Figure 1 with local bottom slope, $\beta$ given by

$$
B=\tan ^{-1}(d \xi / d x)=\tan ^{-1}\left(\xi_{x}\right)
$$

and bottom curvature by $\xi_{x x}$. New length scales associated with variations in water depth must now be considered as discussed by Peregrine (1972). He chose the local bed slope $B$ as the basic parameter to characterize the variable depth. For consistent bottom boundary conditions for the vertical velocity $w$, Peregrine stated it is necessary to assume that

$$
\sigma \geqslant \beta
$$

This means that the local bed slope must always be less than the relative water depth ratio (d/L). This restriction limits the applicability of the Boussinesq equations derived by expansion methods to slowly-varying bathymetry. Using the mean velocity as reference, Peregrine $(1967,1972)$ obtained the following equations for variable depths in two planar dimensions $(x, y)$

Mass

$$
n_{t}+\nabla \cdot[(d+n) u]=0
$$

Motion $u_{t}+(u \cdot \nabla) u+g \nabla \eta=\frac{1}{2}(d) \nabla\left[\nabla \cdot\left(d \cdot u_{t}\right)\right]-\frac{1}{6}\left(d^{2}\right) \nabla\left(\nabla \cdot u_{t}\right)$ 
where the vector operator $\nabla=i \frac{\partial}{\partial \gamma}+j \frac{\partial}{\partial \gamma}+k(0)$. Considering only one-dimensional flow and carefully expanding out the RHS of Equation (21) gives

Mass $\quad \eta_{t}+(d+\eta) u_{x}+u h_{x}=0$

Motion $u_{t}+u u_{x}+g h_{x}-g d_{x}=\frac{1}{3}(d)^{2} u_{x x t}+(d) u_{x t}\left(d_{x}\right)+\frac{1}{2}(d) u_{t}\left(d_{x x}\right)$

For $\epsilon<1, d \approx h$ and the mass equation reduces to Equation (1). Three additional terms appear in Equation (23) due to the water depth variations. Besides the Boussinesq term, the RHS has two extra terms involving bottom slope and curvature which also create local streamline curvature effects in the $\mathrm{x}$ momentum equation. For a horizontal bed, Equation (23) reduces to Equation (2), as expected.

The key aspect of the expansion derivation by Peregrine $(1967,1972)$ or Whitham (1974) is the consistency in the order-of-magnitude of terms retained. All terms of higher order $\left(\epsilon \sigma^{2}, \epsilon^{2}, \sigma^{4}\right.$, etc.) are omitted under the conditions that

$$
\epsilon \approx \sigma^{2} \ll 1
$$

and

$$
\sigma \geqslant B
$$

Other expansion derivations of less rigorous nature (Abbott and Rodenhuis, 1972; Liggett, 1975) obtained additional terms that were products of derivatives and third derivatives in space. Recent applications of the Boussinesq equations for propagation of short (wind) waves in coastal waters (Abbott, Petersen and Skovgaard, 1978; Abbott, 1979) make use of Equations (20) and (21) but in conservation form. Hebenstreit and Reid (1978) use the Eulerian form for numerical investigations of reflection and refraction of solitary waves.

\section{Variation Principles}

A second approach applies variational principles to derive the equations. Early efforts were by Reid and Vastano, 1963; Luke, 1967; and Whitham, 1967. The method essentially proceeds as follows. For the appropriate geometry, boundary conditions and assumptions involved, the kinetic energy per unit mass and integrated over depth (here given the symbol, $T$ ) is determined. Similarly $V$ the potential energy per unit mass and depth integrated is also found. This is usually done for dimensionless terms using a selected set of appropriate scaling parameters.

A Lagrangian $I$ of the form

$$
\mathrm{L}=\mathrm{T}-\mathrm{V}-\lambda(\mathrm{m})
$$

is then introduced where 
$\lambda=$ an undetermined Lagrangian multiplier and

$\mathrm{m}=$ conservation of mass.

Hence, $\lambda(m)$ acts as a constraint upon the system of equations in the derivation. The Euler-Lagrange equations are then employed for the three variables, $n, u$, and $\lambda$.

$\eta: \quad \frac{\partial L}{\partial \eta}+\frac{\partial}{\partial x}\left[\frac{\partial L}{\partial \eta_{x}}\right]+\frac{\partial}{\partial t}\left(\frac{\partial L}{\partial \eta_{t}}\right]_{t}=0$

$u: \quad \frac{\partial L}{\partial u}+\frac{\partial}{\partial x}\left[\frac{\partial L}{\partial u_{x}}\right]+\frac{\partial}{\partial t}\left[\frac{\partial L_{L}}{\partial u_{t}}\right]=0$

$\lambda: \quad \frac{\partial L}{\partial \lambda}+\frac{\partial}{\partial x}\left[\frac{\partial L}{\partial \lambda_{x}}\right]+\frac{\partial}{\partial t}\left[\frac{\partial L_{L}}{\partial \lambda_{t}}\right]=0$

Three equations result from which $\lambda$ can be eliminated and the resulting two equations are the conservation of mass and horizontal momentum.

The first assumption is that the horizontal velocity component (one-dimensional flow) is uniform with depth, then $u=u_{0}$. From the incompressible continuity equation

$$
u_{x}+w_{z}=0
$$

this gives

$$
w=w_{0}-\left(u_{0}\right)_{x} 2
$$

where $w_{0}$ is the integration constant to be evaluated from the boundary conditions with $z$ as defined in Figure 1 . Equation (29) reveals that vertical velocity is a linear variation in depth, 2 . It is sometimes argued that Equation (29) is a separate assumption for the vertical velocity component as a power series in $z$.

$$
\mathrm{w}=\mathrm{w}_{0}+\mathrm{w}_{1} z+\mathrm{w}_{2} z^{2}+\cdots
$$

and truncated to only the first two terms. But in reality, the two assumptions leading to Equation (29) are the uniform horizontal velocity component and that continuity is satisfied. The hierarchy of wave systems created by using successively higher powers of $z$ are discussed by Reid and Vastano (1975).

Local irrotational motion must satisfy the equation

$$
u_{z}-w_{x}=0
$$


and it is clear that the system

$$
u=u_{0} ; \quad w=w_{0}-\left(u_{0}\right)_{x} \cdot z
$$

does not meet this condition. Therefore, the Boussinesq equations have come to be associated with the velocity system of Equation (30) and satisfy continuity but are rotational.

\section{Horizontal bottom}

Whalin (1976) derived equation systems by variational principles for both small amplitude and finite amplitude waves in constant depth. The difference is fundamentally the boundary conditions on the free surface. If $\xi=0$ and $z=0$ at the bottom, then Equation (29) becomes simply

$$
w=-\left(u_{0}\right)_{x} z
$$

Small amplitude theory.--From the exact conservation of mass equation (22)

$$
-\left(u_{0}\right)_{x}=\frac{1}{h}\left(h_{t}+u h_{x}\right)
$$

Small amplitude theory means again assuming $\epsilon=\left(n_{\max } / d\right)<<1$ which means $u_{x}$ is relatively small or of higher order. This gives

$$
w \approx h_{t} \frac{z}{d}
$$

as the approximate vertical velocity profile consistent with the small amplitude assumption. From the variational method, Whalin (1976) obtained the result (dropping the subscript for $u_{0}$ )

Mass:

$$
h_{t}+(u h)_{x}=0
$$

Motion: $\quad u_{t}+\left(g h+\frac{1}{2} u^{2}+\frac{1}{6} h_{t}^{2}+\frac{1}{3} h h_{t t}\right)_{x}=0$

Equation (34) reduces to $(d \approx h$ when $\epsilon \ll 1)$

$$
u_{t}+u u_{x}+g h_{x}=-\frac{1}{3} d h_{x t t}
$$

when products of derivative terms are neglected. Equation (35) and the dimensional equivalent of Equation (17) are identical. The celerity is given by Equation (3) for the linearized forms of Equations (22) and (35). 
Finite Amplitude Theory.--In shallow water, the wave amplitude is not small relative to the water depth. Using equation (32) in (31) and retaining all terms gives

$$
w=\frac{1}{h}\left(h_{t}+u h_{x}\right) 2
$$

as the vertical velocity distribution. Whalin (1976) then obtained the result by variation principles that

Mass:

$$
h_{t}+u h_{x}+h u_{x}=0
$$

Motion:

$$
\begin{aligned}
& u_{t}+\frac{2}{3} h_{x} h_{x t}+\frac{2}{3} h_{x} h_{t t}+\frac{2}{3} u_{t} h_{x}^{2}+2 u h_{x} h_{x t}+u u_{x} \\
& +u_{x} h_{t} h_{x}+\frac{2}{3} u h_{t} h_{x x}+\frac{5}{3} u u_{x} h_{x}^{2}+\frac{4}{5} u^{2} h_{x} h_{x x}+g h_{x} \\
& +\frac{h}{3}\left(u_{x x} h_{t}+3 u_{x} h_{t x}+2 u h_{t x x}+2 u_{x}^{2} h_{x} 2 u u_{x x} h_{x}\right. \\
& \left.+4 u u_{x} h_{x x}+u^{2} h_{x x x}+h_{t t x}+h_{t x} h_{x}+u_{t} h_{x x}\right)=0
\end{aligned}
$$

The full water depth $h$ now appears in the mass Equation (22) in contrast to equation (1) where $h \approx d$. The motion equation is much more complicated. However, if all product of derivative terms and third space derivatives terms are again neglected it reduces to

$$
u_{t}+u u_{x}+g h_{x}=-\frac{1}{3} h h_{t t x}-\frac{2}{3} u h h_{t x x}+\text { H.O.T. }
$$

From Equation (22) it can be shown that

$$
-\frac{1}{3} \mathrm{hh}_{\mathrm{ttx}}-\frac{2}{3}(\mathrm{uh}) \mathrm{h}_{\mathrm{xxt}}=\frac{1}{3} \mathrm{~h}^{2} u_{\mathrm{xxt}}-\frac{1}{3}(\mathrm{uh}) \mathrm{h}_{\mathrm{xxt}}
$$

so that the right-hand-side of (38) could also be written

$$
u_{t}+u u_{x}+g h_{x}=\frac{1}{3} h^{2} u_{x x t}-\frac{1}{3}(u h) h_{x x t}+\text { H.O.T. }
$$

The finite amplitude theory differs from the small amplitude theory in two ways. The total water depth $h$ must be used in the first or primary Boussinesq term and a second Boussinesq term $\left((-1 / 3)\right.$ uhh $\left._{\mathrm{xxt}}\right)$ appears. An important aspect of this investigation is the significance of the second Boussinesq term and all other neglected terms as discussed fully in the section PERTURBATION ANALYSIS. 


\section{Variable water depth}

Mass and Vastano (1978) were interested in tsunami generation and employed variational methods where the bottom varied in both space and time. For purposes of this report, the bottom is assumed fixed in time so that all $\xi_{t}$ terms are omitted from their results. Derivations were made for both small amplitude waves $\left(\epsilon \leqslant \sigma^{2}<<1\right)$ and for finite amplitude, nonlinear waves in which no scaling parameters are involved. the nonlinear forms of the Boussinesqtype equations are summarized here. Again a uniform horizontal velocity and linear variation of the vertical velocity with depth are assumed. Boundary conditions on both the bottom and free surface become nonlinear. At the bottom $(z=\xi)$

$$
w=u \xi_{x}
$$

and at the surface $(z=\xi+h)$

$$
w=n_{t}+u n_{x}
$$

Using Equations (41) in (29) gives

$$
w=\frac{z-\xi}{h}\left(h_{t}+u h_{x}\right)+u \xi_{x}
$$

or

$$
w=-u_{x} z+(u \xi)_{x}
$$

as the vertical velocity distribution. The two equations that result upon elimination of $\lambda(m)$ in the variational method are

Mass:

$$
\eta_{t}+u(n-\xi)_{x}+h\left(u_{x}\right)=0
$$

Motion (Momentun) $I u+J u^{2}+K u_{x}+M u_{t}+N u_{x}+P+Q u_{x x}$

$$
+R u_{x}^{2}+B u_{x t}+A u_{x x}=0
$$

where: $\quad I=2 n_{x} n_{x t}-\frac{1}{2} \xi_{x} n_{x t}+\frac{2}{3} n_{t} n_{x x}-\frac{1}{6} \xi_{x x} n_{t}+\frac{2}{3} h n_{x x t}$

$$
\mathrm{J}=\frac{4}{3} \eta_{\mathrm{x}} \eta_{\mathrm{xx}}-\frac{1}{3} \xi_{\mathrm{x}} \eta_{\mathrm{xx}}+\frac{1}{6} \xi_{\mathrm{xx}} \eta_{\mathrm{x}}-\frac{1}{6} \xi_{\mathrm{xxx}} \xi_{\mathrm{x}}+\frac{1}{3} \mathrm{~h} \eta_{\mathrm{xxx}}+\frac{1}{6} \mathrm{~h} \xi_{\mathrm{xxx}}
$$

$$
\mathrm{K}=\eta_{\mathrm{x}} \eta_{\mathrm{t}}-\frac{1}{2} \xi_{\mathrm{x}} \eta_{\mathrm{t}}+\mathrm{h} \eta_{\mathrm{xt}}
$$

$$
M=\frac{1}{3} h \eta_{x x}+\frac{1}{6} h \xi_{x x}+\frac{2}{3} \eta_{x}^{2}+\frac{1}{6} \xi_{x} \eta_{x}+\frac{1}{6} \xi_{x}^{2}+1
$$

$$
\mathrm{N}=\frac{5}{3} \eta_{x}^{2}-\frac{1}{3} \xi_{x} \eta_{x}-\frac{1}{3} \xi_{x}^{2}+\frac{4}{3} h \eta_{x x}+\frac{2}{3} h \xi_{x x}+1
$$

$$
p=\frac{2}{3} \eta_{t} \eta_{x t}+\frac{2}{3} \eta_{x} \eta_{t t}-\frac{1}{6} \xi_{x} \eta_{t t}+\frac{1}{3} h \eta_{x t t}+g \eta_{x}
$$




$$
\begin{aligned}
& Q=\frac{1}{3} h \eta_{t} \\
& R=A=2 B=\frac{2}{3} h \eta_{x}+\frac{1}{3} h \xi_{x}
\end{aligned}
$$

The nonlinear, variable depth momentum equation now contains a formidable 38 terms! Many are products of derivatives and third space derivations of higher order. Nondimensional scaling of equation (44) revealed that terms of order $\epsilon^{2}, \epsilon^{3}, \epsilon \sigma^{2}$, and $\sigma^{4}$ are retained. Mass and Vastano (1978) state without proof that such a system is appropriate to quasi-long waves with amplitudes as large as one-third the water depth.

A careful reanalysis of these results was made for the further simplification when all products of derivatives and thrid space derivatives are neglected except those involving bottom gradients and curvature. The resulting equation is

$$
\begin{aligned}
u_{t}+u u_{x}+g h_{x}+g \xi_{x}=- & \frac{1}{3} h h_{x t t}-\frac{2}{3} u h h_{x x t} \\
& -\left(\frac{1}{2} h u_{x t}+u h u_{x x}\right) \xi_{x} \\
& -\left(\frac{1}{2} h u_{t}+2 h u u_{x}\right) \xi_{x x}+\text { H.O.T. }
\end{aligned}
$$

For a horizontal bottom, Equation (45) checks with Equation (38), as expected for this nonlinear, finite amplitude case. We can also compare Equation (45) with that derived by Peregrine for small amplitude waves (Equation 23). One additional term appears in both the gradient and bottom curvature terms. Their significance will be shown to be of higher order $O\left(\epsilon \sigma^{2}\right)$ in the section on THEORY. The remaining two terms $\left(1 / 2 h u_{x t}\right.$ and $\left.1 / 2 h u_{t}\right)$ were both found significant by Peregrine except he did not get the $1 / 2$ in the gradient term. The discrepancy was traced back to Equation (44h) where Mass and Vastano (1978) reported that

$$
2 B=\frac{2}{3} h \eta_{x}+\frac{1}{3} h \xi_{x}=\frac{2}{3} h h_{x}+h \xi_{x}
$$

or $\quad B=\frac{1}{3} h_{x}+\frac{1}{2} h \xi_{x}$

It is not possible to determine which version is correct at this time.

Additional applications and a thorough discussion of variational methods appear in whitham (1974) and Mass and Vastano (1978).

Conservation Methods

As will become evident in what follows, the third derivational approach is given the label "conservation methods." 
Beginning in 1974, applied mathematicians and engineering mechanicians, knowledgeable of classical, non-linear theories of plates and shells in solid mechanics, began applying these same ideas to water wave theory (Green, Laws, and Naghdi, 1974). Their motivation was to avoid the formal expansion method and establish the required system of equations without approximations. The direct formulation approach by cosserat and cosserat (1909) used in the development of shell theory was adapted. Consequently, theoretical derivations for jet-like and sheet-like fluid flows were developed and results published in a series of papers. The derivation of equations for water wave propagation in variable depths is presented in Green, Laws, and Naghdi, 1974; Green and Naghdi, 1976a; 1976b; and summarized by Naghdi (1978). They call it the method of directed fluid sheets.

The method begins with the known differential equations of continuity and energy in three-dimensions for a homogeneous, incompressible and inviscid fluid. Two assumptions are then made. The whole fluid system is required to be invariant under an assumed rigid-body translation (Galilean invariant). And, an approximation for the velocity field is made which satisfies the continuity equation. Using this information, the exact boundary conditions at the free surface and at the bed, and the energy equation in integral form, the system of equations for water waves over variable bathymetry is derived, without making further approximations. Throughout their work the velocity field is assumed to be uniform with depth in the horizontal directions and the vertical velocity varies linearly with the vertical, as in all previous methods discussed above. Thus the flow is rotational in horizontal planes as normally occurs in shallow water with large wave amplitudes relative to water depth. Further details of the method of directed fluid sheets are beyond the scope of this report.

For a horizontal bottom, Naghdi (1978) presents the equations

Mass:

$$
n_{t}+(d+n) u_{x}+u n_{x}=0
$$

Motion: $\quad u_{t}+u_{x}+g n_{x}+\frac{1}{3} d \eta_{t t x}=R$

same as (22)

$$
\begin{aligned}
& R=-\frac{1}{3} n \eta_{t t x}-\frac{2}{3} h_{x} h_{t t}-\frac{1}{3} \frac{1}{h}[ h^{2}\left(2 u h_{x t}+u^{2} h_{x x}+\right. \\
&\left.\left.u_{t} h_{x}+u u_{x} h_{x}\right)\right]_{x}=0
\end{aligned}
$$

where

For small amplitude waves $(\epsilon<<1), R$ is neglected and Equation (48) reduces to that found by Peregrine (1967) from expansion methods (Equations 14 or 17) and by Walin (1976) and Mass and Vastano (1978) by variational methods (Equation 35). This is also the original formulation of Boussinesq (Equation 2). It may be concluded that the small amplitude theory is not invariant under a constant, superimposed rigid body translation.

With $R$ included the equations are nonlinear and invariant under rigid body translation. Naghdi (1978) states that within the scope of a nonlinear theory, the quantity $\mathrm{R}$ should not be neglected on either physical or mathemaical grounds. However, if Equation (48) is carefully expanded out and all product of derivative terms and third space derivatives are neglected, the 
equation of motion is identical to Equation (40) as determined from variational methods under these assumptions. The central question again reduces to the size of the two Boussinesq terms in Equation (40) relative to additional terms resulting from derivative products and third derivatives.

A similar conservation method has been put forth by Venezian (1974, 1975). Integral forms of the conservation equations of mass, momentum and energy are obtained by integrating across the vertical from bottom to free surface. An assumed velocity field is then substituted into these conservation equations so that continuity is satisfied but not irrotationality. Waves are permitted to travel in both directions and are Galilean invariant. Consequently, the method appears identical to what was presented by Naghdi (1978) but much easier to follow. For this reason we have chosen to present a detailed review of this derivational approach in the section on THEORY.

In Eulerian form on a horizontal bed, Venezian (1974) obtained the usual mass equation as Equation (22) and for the motion equation

$$
\begin{aligned}
u_{t}+u u_{x}+g h_{x} & +h h_{x}\left(u_{x}^{2}-u_{x t}-u u_{x x}\right. \\
& +\frac{1}{3} h^{2}\left(u_{x} u_{x x}-u_{x x t}-u u_{x x x}\right)=0
\end{aligned}
$$

The horizontal velocity was again taken as uniform with depth, giving a linear, vertical velocity distribution for which the full nonlinear free surface boundary conditions were employed (Equation 36). Surprisingly, if product of derivative and third space derivative terms are neglected in Equation (49), the result reduces to the Boussinesq form for small amplitude theory and not to Equation (40) for finite-amplitude waves. The term $-\left(\frac{1}{3}\right)$ uhh ${ }_{\text {xxt }}$ does not appear. Further reasons for this result are explored in the section on THEORY where the equations in conservation form are presented along with those for variable water depths.

\section{Summary of Equations}

The basic equations of motion from the three derivational methods are summarized in Table 1. Conservation of mass is given by Equation (22). For small amplitude waves $\left(\epsilon \approx \sigma^{2}<<1\right)$ the equations of motion derived by Peregrine $(1967,1972)$ and Whitham $(1965,1974)$ are identical to $O\left(\epsilon^{2}, \epsilon \sigma^{2}, \sigma^{4}\right)$. For finite amplitude waves, both variational methods (Whalin, 1976; Mass and Vastano, 1978) and the conservation method of Naghdi (1978) give two identical Boussinesq terms plus 16 additional terms consisting of products of derivatives and third space derivatives. It is not known, at this stage, if the 16 added terms are identical by both derivational methods. The method by Venezian $(1974,1975)$ in Eulerian form produced only a single Boussinesq term and five additional higher-order-terms on a horizontal bed. The equation given by Berlamont (1976) from his work on roll waves is also shown in Table 1. The derivational method is unknown. Three Boussinesq terms are said to be important.

For variable water depths, the results are limited. Except for the $1 / 2$ factor in one term as previously discussed, the results of Peregrine (1967) 


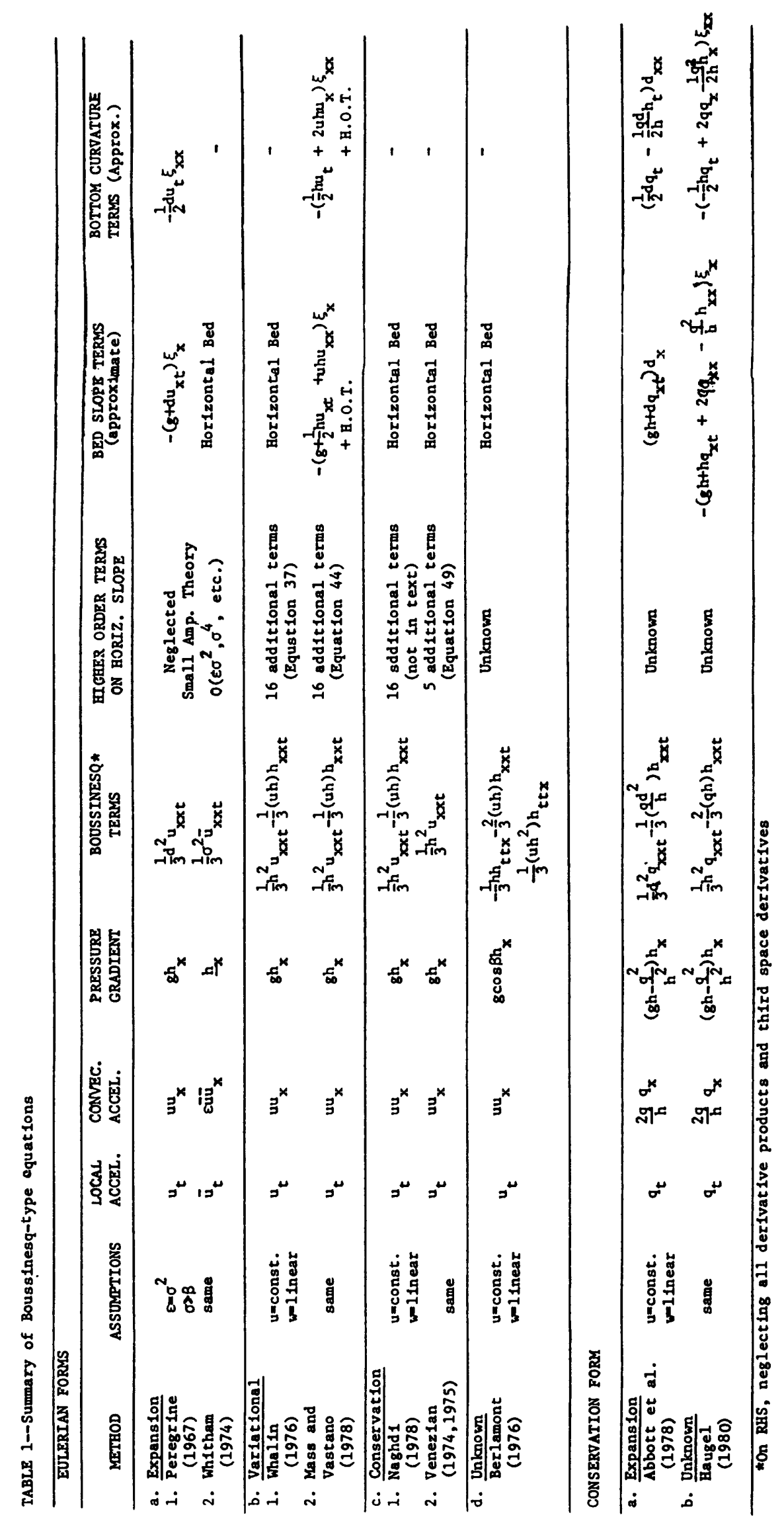


and Mass and Vastano (1978) agree to the order of small amplitude theory. For finite amplitude theory, an additional 12 gradient and curvative terms

involving derivative product and third space derivatives were obtained by Mass and Vastano (1978).

Two references with Boussinesq-type equations given in Conservative Form were studied and are also shown in table 1. They will be discussed further in the section on THEORY.

\section{Arbitrary Cross Sections}

All the equations examined above are for one-dimensional flow in wide, rectangular channels where the local water depth and hydraulic radius are identical. This limitation is expedient for research purposes since the importance of the higher order terms is still questionable, especially for variable water depths. For practical future applications, equations must eventually be derived for arbitrary cross sections. Three approaches are possible. At one extreme, the three-dimensional, incompressible motion equations with proper turbulence models can be used for appropriate boundary conditions. This is the most expensive approach but may be justified for highly irregular and curved boundaries in important cases.

The two-dimensional Boussinesq equations could also be used for relatively wide river sections where vertical, depth-averaging is a good approximation. These equations are presently being used to simulate short wave phenomena along coasts and within harbors (Abbott, Petersen, and Skovgaard, 1978; Abbott, 1979). Additional cross momentum Boussinesq terms appear in each equation.

A third approach is to derive Boussinesq-type equations for a prismatic channel of arbitrary cross-section. Such an approach was taken by Peregrine (1968) who again used expansion methods in the derivation. At this level of approximation, both surface amplitude and horizontal velocity vary over the cross-section of the channel. The variation of cross-section with surface elevation must also be included. Application of the method is summarized in Peregrine (1976) and used by Berlamont (1976). Details are beyond the scope of this report since the question of appropriate equations for even rectangular sections remains.

Finally, Dressler (1978) derived generalized, nonlinear, shallow-water equations which explicitly include bottom curvature effects. The equations account for bathymetric variations on the pressure distribution, velocities, and free-surface profile and reduce to the long wave equations of Saint Venant for constant slopes on horizontal bottoms. But, they do not include wave-like variations of the free-surface to simulate short waves or rapidly-varied flows that create frequency dispersive effects like the Boussinesq equations. The derivation method utilizes curvilinear coordinates and should be extended to derive equations that reduce to Boussinesq-type equations on horizontal beds. The usefulness of the equations by Dressler (1978) is greatly diminished for lack of appropriate Boussinesq terms. 


\section{THEORY}

It is instructive to follow the derivation method of venezian (1974, 1975), but to rederive the Boussinesq equations in Conservation Form. Venezian's approach is easier to follow than the other methods. Solutions of Conservation Form equation systems are more general in that both continuous and discontinuous flows (i.e., shocks such as hydraulic jumps, bores, and breaking waves) are automatically obtained (Abbott, 1979). It is often near such discontinuous disturbances that the free surface is most rapidly curved requiring Boussinesq-type equations to describe the motion.

\section{Conservation Form Following Venezian}

Consider one-dimensional flow in a wide, rectangular channel on a horizontal bed. The incompressible continuity equation is given by

$$
u_{x}+w_{z}=0
$$

and the Euler equations of motion for essentially two-dimensional motion are

$x$-Direction $\quad u_{t}+u_{x}+w u_{z}=-\frac{1}{\rho} p_{x}$

$$
\text { z-Direction } \quad w_{t}+u w_{x}+w_{z}=-\frac{1}{\rho} p_{z}-g
$$

where now $u, w$ are the local velocity components, and $p$, the local pressure. The fluid is assumed homogeneous of density, $\rho$. bed

For a solution, the following boundary conditions are required. At the

$$
z=0 \text {, so that } w=0
$$

On the free surface, both kinematic and dynamic boundary conditions must be considered. The surface air pressure is assumed constant and locally atmospheric so that dynamically

$$
p_{s}=0
$$

and kinematically

$$
h_{t}+u_{s} h_{x}=w_{s}
$$

must hold from conservation of mass considerations. The subscript $s$ means on the surface. Equations (52), (53), and (54) are the needed boundary conditions.

The energy equation corresponding to Equations (50) and (51) with no momentum diffusing terms present can be obtained by multiplying Equation (50) by $u$ and Equation (5l) by $w$ and adding the results giving

$$
\left[1 / 2\left(u^{2}+w^{2}\right)\right]_{t}+u\left[1 / 2\left(u^{2}+w^{2}\right)\right]_{x}+w\left[1 / 2\left(u^{2}+w^{2}\right)\right]_{z}=-\frac{1}{\rho}\left[u p_{x}+w p_{z}\right]-g w
$$


No energy loss terms appear in Equation (55) and all equations are in their primitive form. For completeness the vorticity, defined as

$$
\omega=w_{x}-u_{z}
$$

will give after differentiating Equation (50) by $z$ and Equation (51) by $x$

$$
\omega_{t}+u \omega_{x}+w w_{z}=0
$$

\section{Conservation Integrals}

The above differential equations for mass, momentum, energy and vorticity hold locally at any point $(x, z, t)$ in the flow. Venezian (1974) derived corresponding conservation integrals for these four quantities over the vertical water column from the bed to the free surface $(z=h)$. Use was made of the fact that for any function $f(x, z, t)$ the following equality holds based on Leibnitz' rule

$$
\left[\int_{0}^{h} f d z\right]_{t}+\left[\int_{0}^{h} f u d z\right]_{x}=\int_{0}^{h}\left[f_{t}+(f u)_{x}\right] d z+f_{s}\left(h_{t}+u_{s} h_{x}\right)
$$

where $f_{s}$ is the value of $f$ at the free surface. Expanding the first term on the right-hand-side of Equation (58), integrating the term $\int_{0}^{h}\left(f u_{x}\right) d z$ by parts
and using the continuity Equation $(28)$ gives

$$
\left.\int_{0}^{h} f d z\right]_{t}+\left[\int_{0}^{h} f u d z\right]_{x}=-f_{s} w_{s}+\int_{0}^{h} w f_{z} d z+f_{s}\left(h_{t}+u_{s} h_{x}\right)+\int_{0}^{h}\left[f_{t}+u f_{x}\right] d z
$$

But, from the kinematic free surface boundary condition given by Equation (54), the surface terms cancel leaving

$$
\left[\int_{0}^{h} f d z\right]_{t}+\left[\int_{0}^{h} f u d z\right]_{x}=\int_{0}^{h}\left(f_{t}+u f_{x}+w f_{z}\right) d z
$$

Equation $(60)$ is the fundamental conservation integral over the water column for any $f(x, z, t)$. The quantity $f$ is on a unit mass (density) basis.

Continuity integral

When $f=1$, Equation $(60)$ becomes the conservation integral for continuity.

$$
\left[\int_{0}^{h} d z\right]_{t}+\left[\int_{0}^{h} u d z\right]_{x}=0
$$


Horizontal momentum integral

Taking $f=u$ using Equation (50) gives

$$
\left[\int_{0}^{h} u d z\right]_{t}+\left[\int_{0}^{h} u^{2} d z\right]_{x}=-\frac{1}{\rho} \int_{0}^{h} p_{x} d z
$$

but

$$
\left[\int_{0}^{h} p d z\right]_{x}=p_{s} h_{x}+\int_{0}^{h} p_{x} d z
$$

and from Equation (53), $p_{8}=0$ gives

$$
\left[\int_{0}^{h} u d z\right]_{t}+\left[\int_{0}^{h}\left(u^{2}+p / \rho\right) d z\right]_{x}=0
$$

Equation (62) is the conservation integral for linear momentum.

\section{Energy integral}

The total energy per unit mass is composed of kinetic and potential energy, hence $f=k\left(u^{2}+w^{2}\right)+g z$. Using this for $f$ in Equation (60) plus the energy (55) and continuity (28) equations and boundary conditions gives

$$
\left[\int_{0}^{h}\left\{\frac{1}{2}\left(u^{2}+w^{2}\right)+g z\right\} d z\right]_{t}+\left[\int_{0}^{h}\left\{\frac{1}{2}\left(u^{2}+w^{2}\right)+g z+p / \rho\right\} u d z\right]_{x}=0
$$

as the conservation integral for energy.

\section{Vorticity integral}

Setting $f=w$ in Equation (60) plus making use of Equation (57) for vorticity gives

$$
\left[\int_{0}^{h} \omega d z\right]_{t}+\left[\int_{0}^{h} \omega u d z\right]_{x}=0
$$

as the conservation integral for vorticity.

In summary, for the four quantities on a per unit mass basis

$$
f=\left\{\begin{array}{cl}
1 & \text { mass } \\
u & \text { momentum } \\
\frac{1}{2}\left(u^{2}+w^{2}\right)+g z & \text { energy } \\
w & \text { vorticity }
\end{array}\right.
$$


we may write the conservation integral in general as

$$
\left[\int_{0}^{h} D d z\right]_{t}+\left[\int_{0}^{h} F d z\right]_{x}=0
$$

where D the "density" per unit width is

$$
D=\left\{\begin{array}{cl}
1 & \text { mass } \\
u & \text { momentum } \\
\frac{1}{2}\left(u^{2}+w^{2}\right)+g z & \text { energy } \\
w & \text { vorticity }
\end{array}\right.
$$

and the "flux" per unit width, $F$ is given by

$$
F= \begin{cases}u & \text { mass } \\ u^{2}+p / p & \text { momentum } \\ {\left[\frac{1}{2}\left(u^{2}+w^{2}\right)+g z+p / p\right] u} & \text { energy } \\ w u & \text { vorticity }\end{cases}
$$

to give conservation of mass, momentum, energy and vorticity. Laws expressed in the form of Equation (66) [i.e. Equations (61), (62), (63), and (64)] are said to be in Conservation Form since the quantities mass, momentum, energy and vorticity are transported in time and space, not pressure (water depth) and velocity. Whitham (1974) outlines prodedures to construct an infinity of further conservation forms. We are only interested in the above four since they have physical significance.

Equations (66) are exact for ideal fluids.

\section{Long Wave Propagation}

Integration of Equations (66) requires assumptions for $u$ and $p$ over the water column. If a hydrostatic pressure distribution is assumed for $p$ and $u$ is assumed uniform over the water depth, the classic long wave, shallow water equations of saint-venant for mass and momentum are obtained in conservation form.

Mass

$$
h_{t}+q_{x}=0
$$

Motion

$$
q_{t}+\left[\frac{q^{2}}{h}+\frac{g h^{2}}{2}\right]_{x}=0
$$


where $q=$ uh is introduced to distinguish the conservation form. The dependent variables are now $h$ and $q$ in time and space. Expanding out Equation (68) gives an alternate form shown in Table 1.

$$
q_{t}+2 \frac{q}{h} q_{x}+\left(g h-\frac{q^{2}}{h^{2}}\right) h_{x}=0
$$

Very long waves exhibit essentially negligible streamline curvature so that the hydrostatic pressure distribution assumption is valid.

\section{Quasi-Long Wave Propagation}

The pressure distribution assumption is now relaxed and omitted in what follows. The horizontal velocity is again assumed constant in $z$

$$
u=u(x, t)
$$

which together with the continuity equation results in a vertical velocity linear with depth

$$
w=-u_{x} \cdot z
$$

or

$$
w=\frac{1}{h}\left(h_{t}+u h_{x}\right) \cdot z
$$

This assumption for $\mathrm{u}$ is the only assumption made. Using Equation (70) in the mass conservation (continuity) integral Equation (61) again gives Equation (67). Substituting Equation (70) in the momentum integral Equation (62) gives

where

$$
\begin{aligned}
& q_{t}+\left[\frac{q^{2}}{h}+B\right]_{x}=0 \\
& B=\frac{1}{\rho} \int_{0}^{h} p d y
\end{aligned}
$$

and $B$ is a third unknown in the problem*. Clearly, a third independent equation is required.

Venezian (1974) used the energy equation to derive an explicit expression for B. In a later paper, Venezian (1976) derived the identical expression for $B$ from the vertical momentum integral equation. The final form of the resulting expression from the latter method is (Venezian, 1976)

$$
B=\left[\int_{0}^{h} w z d z\right]_{t}+\left[\int_{0}^{h}(u \cdot w) z \cdot d z\right]_{x}-\int_{0}^{h} w^{2} d z+\frac{1}{2} g h^{2}
$$

*B is obviously a different variable than used previously 
Equation (73) was said to be first derived by starr (1947) and verified by Longuet-Higgins (1974). Use of the vertical momentum integral equation and hence Equation (73) to determine $B$ is the more general approach. The energy integral, equation (63), contains the term

$$
\frac{1}{\rho} \int_{0}^{h} p u d z
$$

for which $u$ constant in $z$ becomes simply $B \cdot u$. Use of the energy equation to find $B$ is only feasible for this assumption on $u$. For higher order approximations with $u$ variable in $z$, equation $(73)$ is the only way to find $B$, explicitly.

Using Equations (70) and (30) in Equation (73) gives

$$
\begin{aligned}
B & =\left[\int_{0}^{h}\left(-z^{2} u_{x}\right) d z\right]_{t}+\left[\int_{0}^{h}\left(-z^{2} u u_{x}\right) d z\right]_{x}-\int_{0}^{h} z^{2} u_{x}^{2} d z+\frac{1}{2} g h^{2} \\
& =-\left[\frac{1}{3} h^{3} u_{x}\right]_{t}-\left[\frac{1}{3} h^{3} u u_{x}\right]_{x}-\frac{1}{3} h^{3} u_{x}^{2}+\frac{1}{2} g h^{2}
\end{aligned}
$$

or

$$
B=\frac{1}{2} g h^{2}-\frac{1}{3} h^{3}\left(u_{x t}+u_{x x}+2 u_{x}^{2}\right)-h^{2}\left(h_{t} u_{x}+h_{x} u_{x}\right)
$$

But from continuity, the last term in Equation (75) can be written

$$
-h^{2}\left(h_{t} u_{x}+h_{x} u_{x}\right)=-h^{2}\left(-h u_{x}^{2}\right)
$$

so that

$$
B=\frac{1}{2} g h^{2}-\frac{1}{3} h^{3}\left(u_{x t}+u u_{x x}-u_{x}^{2}\right)
$$

\section{Eulerian form}

Venezian (1974) expanded Equations (71) using $q=$ uh and continuity to give

$$
h\left(u_{t}+u u_{x}\right)+B_{x}=0
$$

which by expanding equation (76), becomes

$$
\begin{aligned}
h\left(u_{t}+u u_{x}\right) & +g h h_{x}+h^{2} h_{x}\left(u_{x}^{2}-u_{x t}-u u_{x x}\right) \\
& +\frac{1}{3} h^{3}\left(u_{x} u_{x x}-u_{x x t}-u u_{x x x}\right)=0
\end{aligned}
$$

Dividing through by the common factor $h$ gives Equation (49). This reduces to the Boussinesq equation for small amplitude waves for a horizontal bottom when 
neglecting derivative products and third space derivatives. The term $-\left(\frac{1}{3}\right)$ uh $h_{\text {xxt }}$ found by Mass and Vastano (1978), Whal in (1976), and Naghdi (1978) does not appear (Table 1). The reason why is not clear. One possibility is that the five additional terms in Venezian's derivation are equivalent to the 16 added terms plus this second Boussinesq term in these other derivations. This question is addressed further in section PERTURBATION ANALYSIS.

\section{Conservation forms}

Using $u=q / h$ in Equation (76) gives for the motion equation in conservation form

$$
q_{t}+\left\{\frac{q^{2}}{h}+\frac{1}{2} g h^{2}-\frac{1}{3} h^{3}\left[\left(\frac{q}{h}\right]_{x t}+\left[\frac{q}{h}\right]\left[\frac{q}{h}\right]_{x x}-\left(\left[\frac{q}{h}\right]_{x}\right]^{2}\right]\right\}_{x}=0
$$

Again neglecting derivative products and third space derivatives, this becomes after expanding

$$
q_{t}+\left(\frac{2 q}{h}\right) q_{x}+\left(g h-\frac{q^{2}}{h^{2}}\right) h_{x}=\frac{1}{3} h^{2} q_{x x t}-\frac{1}{3}(q h) h_{x x t}
$$

Equation (79) is identical to that used by Abbott, Petersen and Skovgaard (1978) if $d \approx h$ (Table 1) and on a horizontal bed.

Haugel (1980) stated without derivation the following motion equation

$$
q_{t}+\left(\frac{q^{2}}{h}\right)_{x}+\left\{\left[\left(\frac{g+\beta}{2}\right)+\frac{\alpha}{3}\right] h^{2}\right\}_{x}=-\left(g+\beta+\frac{\alpha}{2}\right] h \xi_{x}
$$

where the terms $\alpha$ and $\beta$ characterize vertical accelerations due to both wave steepness and bed slope effects. They were defined as

$$
\begin{aligned}
& \beta=\frac{d^{2} \xi}{d t^{2}} \\
& \alpha=\frac{d^{2} h}{d t^{2}}
\end{aligned}
$$

where the total (substantive) derivative is

$$
\frac{\mathrm{d}}{\mathrm{dt}}=\frac{\partial}{\partial t}+\left[\frac{\mathrm{q}}{\mathrm{h}}\right] \frac{\partial}{\partial \mathrm{x}}
$$

After considerable expansion in which all derivative product terms were neglected, Equation ( 80 ) becomes for a horizontal bottom

$$
q_{t}+2\left(\frac{q}{h}\right) q_{x}+\left(g h-\frac{q^{2}}{h^{2}}\right) h_{x}=\frac{1}{3} h^{2} q_{x x t}-\frac{2}{3}(q h) h_{x x t}
$$


The last term in Equation (82) appears wrong due to the factor of 2. Since Equation $(80)$ is stated as fact with no derivation presented, it is impossible to determine why the added factor 2 appears. It is assumed incorrect for this report.

In all cases however, two additional Boussinesq terms appear in conservation form. This is because in Equation (78)

$$
\begin{aligned}
\frac{1}{3} h^{3}\left[\left[\frac{q}{h}\right]_{x t}\right]_{x} & =\frac{1}{3} h^{3}\left(\frac{q}{h}\right]_{x x t} \\
& =\frac{1}{3} \frac{h^{3}}{h} q_{x x t}-\frac{1}{3} \frac{h^{3} q}{h^{2}} h_{x x t} \\
& =\frac{1}{3} h^{2} q_{x x t}-\frac{1}{3}(q h) h_{x x t}
\end{aligned}
$$

and all other terms were neglected. When $u$ and $h$ are the dependent variables, this second Boussinesq term automatically disappears. Consequently, the appearance of a second Boussinesq term in the conservation Form equations should not be confused with a similar looking term in the Eulerian form equations derived by variational principles and other methods. variables

Venezian (1976) obtained an alternate Conservation form in terms of $u, h$

$$
\left[u+\frac{1}{6} h^{2} u_{x x}\right]_{t}+\left[g h+\frac{1}{2} u^{2}-\frac{1}{2} h^{2}\left[u_{x t}+\frac{2}{3} u u_{x x}-u_{x}^{2}\right)\right]_{x}=0
$$

His results were shown to be an extension of the work of Benjamin and Lighthill (1954) to the time-dependent equations. The resulting equations permit wave propagation in two directions and are invariant under Galilean transformation. This latter property is also true for the derivations by variational principles and Naghdi (1978) by directed fluid sheets. In this theory, continuity is satisfied microscopically but irrotationality is not. Additional velocity distributions can be used in the conservation integrals to derive higher order equations but are beyond the scope of this report.

\section{Nondimensional Equations}

The equations derived by Venezian (1974, 1976), Equations (47) and (49), are now made dimensionless to study the relative order-of-magnitude of individual terms. A set of scaling parameters is employed that were first introduced by Hammack (1973) and utilized by Mass and Vastano (1978). Dimensional quantities are here taken as starred quantities (i.e. all terms in Equations (47) and (49) would appear with star superscripts) and defined as

$$
\begin{aligned}
& \mathrm{x*}=\mathrm{xL} \\
& t *=t L^{*} / \mathrm{C} * \\
& \mathrm{u} * \mathrm{u} \in \mathrm{C} * \\
& \mathrm{~h} * \mathrm{hd}
\end{aligned}
$$

so that $q *=u * h *=(u h) \epsilon C * d *$, and 
where:

$$
\begin{aligned}
& c *=\sqrt{g d^{*}}, \text { the celerity of shallow water disturbance, } \\
& \epsilon=\eta_{\max }^{*} / d^{*} \text {, the relative wave height, and } \\
& \sigma=d^{*} / L^{*}, \text { the relative water depth. }
\end{aligned}
$$

All corresponding nondimensional variables $(u, h, x, t)$ use the same symbol without asterisk and have magnitudes of the order unity.

Table 2 lists all the derivatives appearing in the continuity and motion equations discussed above and their dimensionless form. The coefficients then give a scale to each term.

\section{Mass Conservation}

Rewriting Equation (47) as

$$
h_{t}^{*}+h^{*} u_{x}^{*}+u^{*} h_{x}^{*}=0
$$

and making the appropriate substitutions from Equation (85) and Table 2 gives after dividing through by $\mathrm{co}$

$$
h_{t}+(\epsilon) h u_{x}+(\epsilon) u_{x}=0
$$

The time derivative is dominant and both space derivatives are of order $(\epsilon)$ which is not the same result as Equation (13) where the parameter scaling was part of the derivation.

Table 2 Nondimensional derivative terms in fundamental equations

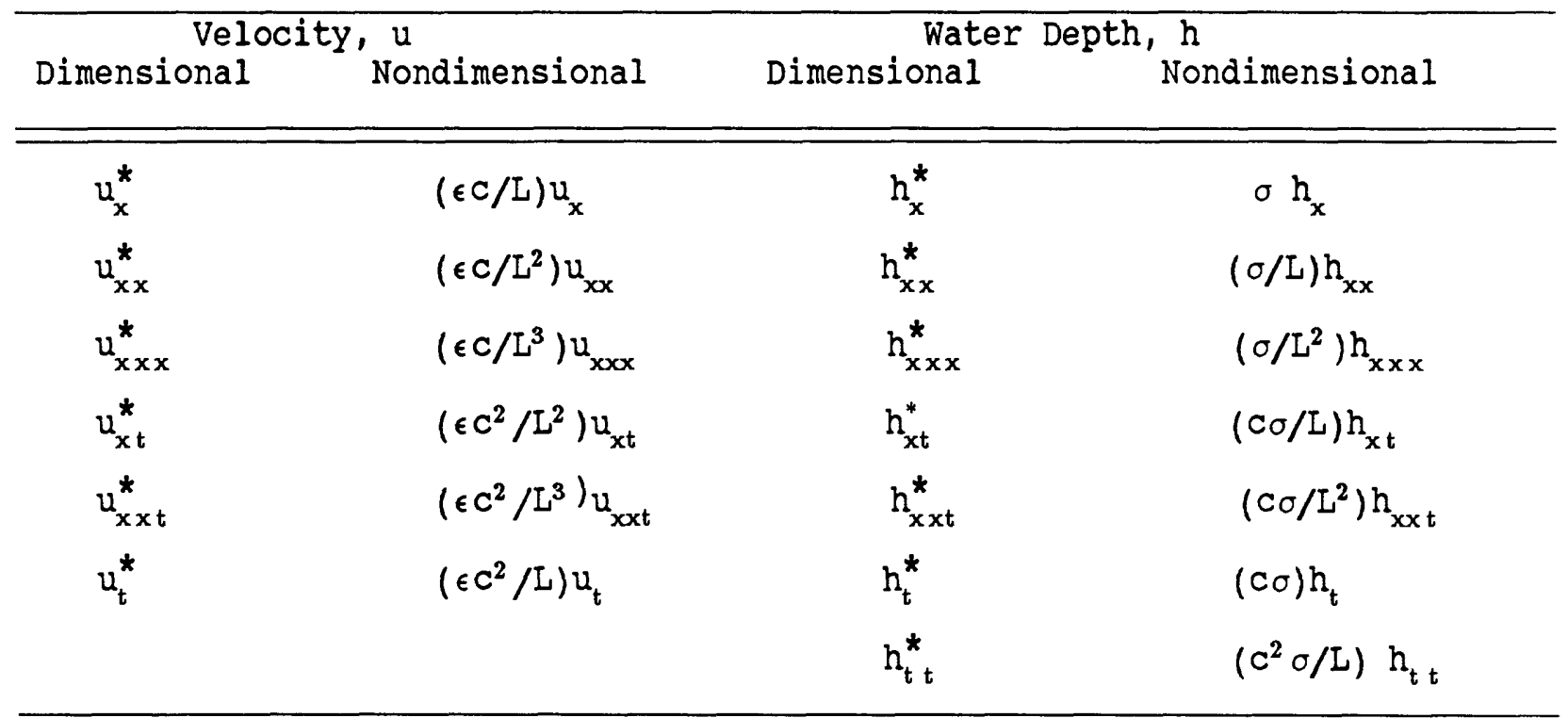

where both $\mathrm{C}$ and $\mathrm{L}$ are dimensional quantities but the asterisk is dropped for convenience. 


\section{Momentum Conservation}

Starting with Venezian's results, Equation (49) rewritten here as

$$
\begin{gathered}
u_{t}^{*}+u^{*} u_{x}^{*}+g^{*} h_{x}^{*}+h^{*} h_{x}^{*}\left[\left(u_{x}^{*}\right)^{2}-u_{x t}^{*}-u^{*} u_{x x}^{*}\right] \\
+\frac{1}{3} h^{* 2}\left[u_{x}^{*} u_{x x}^{*}-u_{x x t}^{*}-u^{*} u_{x x x}^{*}\right]=0
\end{gathered}
$$

the appropriate values are again replaced giving

$$
\begin{aligned}
& \left(\epsilon C^{2} / L\right) u_{t}+\left(\epsilon^{2} c^{2} / L\right) u_{x}+g^{*} \sigma h_{x}+ \\
& (d \sigma)\left(h h_{x}\right)\left[\left(\epsilon^{2} c^{2} / L\right\} u_{x}^{2}-\left(\epsilon C^{2} / L^{2}\right) u_{x t}-\left(\epsilon^{2} c^{2} / L^{2}\right) u_{x x}\right] \\
& +\frac{1}{3} d^{2} h^{2}\left[\left(\epsilon^{2} c^{2} / L^{3}\right) u_{x} u_{x x}-\left(\epsilon C^{2} / L^{3}\right) u_{x x t}-\left(\epsilon^{2} c^{2} / L^{3}\right) u_{x x x x}\right]=0
\end{aligned}
$$

Dividing through by $\epsilon \mathrm{C}^{2} / \mathrm{L}$ gives

$$
\begin{aligned}
u_{t}+ & (\epsilon) u u_{x}+\left(\frac{g^{*} \sigma L}{\epsilon C^{2}}\right) h_{x}+(d \sigma) h h_{x}\left[(\epsilon) u_{x}^{2}-(t) u_{x t}-(\epsilon) u_{x x}\right] \\
& +\frac{1}{3} d^{2} h^{2}\left[\left(\frac{\epsilon}{L^{2}}\right) u_{x} u_{x x}-\left(\frac{1}{I^{2}}\right) u_{x x t}-\left(\frac{\epsilon}{L^{2}}\right) u u_{x x x}\right]=0
\end{aligned}
$$

or since $\sigma=d / L, \sigma^{2}=d^{2} / L^{2}$ and letting $g=g * /\left(c^{2} / L\right)$

$$
\begin{aligned}
u_{t}+ & (\epsilon) u u_{x}+\left(\frac{\sigma}{\epsilon}\right) g h_{x}+h h_{x}\left[\left(\epsilon \sigma^{2}\right) u_{x}^{2}-\left(\sigma^{2}\right) u_{x t}-\left(\epsilon \sigma^{2}\right) u_{x x x}\right] \\
& +\frac{1}{3} h^{2}\left[\left(\epsilon \sigma^{2}\right) u_{x} u_{x x}-\left(\sigma^{2}\right) u_{x x t}-\left(\epsilon \sigma^{2}\right) u_{x x x}\right]=0
\end{aligned}
$$

Rearranging Equation (89) gives

$$
u_{t}+(\epsilon) u u_{x}+\left(\frac{\sigma}{\epsilon}\right) g h_{x}=+\frac{1}{3}\left(\sigma^{2}\right) h^{2} u_{x x t}+\left(\sigma^{2}\right) h h_{x} u_{x t}+o\left(\epsilon \sigma^{2}\right)
$$

The dominant pressure term gives rise to local accelerations with the convective acceleration and Boussinesq term of lower magnitude but equally important when $\epsilon \approx \sigma^{2}$. An additional term of $O\left(\sigma^{2}\right)$ appears that comes from one derivative product. All other derivative products or third space derivatives are of $O\left(\epsilon \sigma^{2}\right)$ and smaller. The result that one derivative product term is of $O\left(\sigma^{2}\right)$ is surprising.

As shown in Table 1 and discussed above, other derivational methods obtained a second Boussinesq term of the form

$-\frac{1}{3}(u * h *) h_{x \times t}^{*}$ 
which by the same procedure becomes in dimensionless form

$$
-\frac{1}{3}\left(\sigma^{2}\right)(u h) h_{x x t}
$$

This second Boussinesq term is of the same order-of-magnitude as the primary term by this scaling procedure. Similarly, the sixteen additional terms found by Whalin (1976) and given in Equation (37) were analyzed. It was discovered that two are of $O\left(\sigma^{2} / \epsilon\right)$, seven are of $O\left(\sigma^{2}\right)$, and the remaining seven of higher order $O\left(\epsilon \sigma^{2}\right)$. Consequently, it can be concluded from results of the scaling procedure, that all derivative product terms and third space derivatives are not of the same order-of-magnitude. And, more importantly, that many are equally as important as the primary Boussinesg term for finite-amplitude wave propagation. Obviously, their importance will greatly diminish for small amplitude waves.

other scaling variables are possible. Meyer (1967) presents a detailed mathematical discussion of the relative sizes of various scaling factors.

\section{Bottom Variation Scaling}

As discussed earlier, Peregrine (1972) showed that the local bed slope must always be less than the relative water depth ratio, or $\sigma \geqslant \beta$. This restriction was to keep the vertical velocity near the bed consistent in magnitude with the order of the derivation. The finite-amplitude wave derivations by variational methods and the directed fluid sheet method relaxed this restriction.

\section{Bed gradient terms}

Table 1 shows three additional bed slope terms from the variational method of Mass and Vastano (1978). One is due to the fluid weight component, $\left(g \xi_{x}\right)$, appears in the long wave equations, and will not be discussed further. If the above procedures are applied to the remaining two terms, the result is, in nondimensional terms,

$$
\cdots-(\sigma \beta) \frac{1}{2} h u_{x t}-(\sigma \in \beta) \text { uhu } u_{x x}
$$

Clearly, the second term in expression (92) is of higher order if we take $\sigma \geqslant \beta$ and these results then confirm Peregrine's results (except for the $\frac{1}{2}$ factor). product of derivative terms involving $\xi_{x}$ were all neglected in Table 1 .

\section{Bottom curvature terms}

The two terms obtained by Mass and Vastano (1978) (Table 1) were put in nondimensional form with the result

$$
\cdots-\left(\sigma \dot{\beta}_{x}\right) \frac{1}{2} h u_{t}-\left(\epsilon \sigma \beta_{x}\right) h u u_{x}
$$


The second term is again of higher order and this result also was obtained by Peregrine (1967, 1972).

\section{Summary, Nondimensional Equations}

We may summarize the above nondimensional equations as follows:

Mass: $\quad h_{t}+(\epsilon) u h_{x}+(\epsilon) h u_{x}=0$

Motion: $u_{t}+(\epsilon) u_{x}+\left(\frac{\sigma}{\epsilon}\right) g h_{x}+(\beta) g=$

$$
\begin{aligned}
& +\left(\sigma^{2}\right) \frac{1}{3} h^{2} u_{x x t}-\left(\sigma^{2}\right) \frac{1}{3}(u h) h h_{x x t} \\
& -(\sigma \beta) h u_{x t}-\left(\sigma \beta_{x}\right) \frac{1}{2} h u_{t} \\
& +\left(\sigma^{2}\right) \text { (additional terms) }+O\left(\epsilon \sigma^{2}, \epsilon^{2}, \sigma^{4}\right. \text {, etc.) }
\end{aligned}
$$

All terms of order $\epsilon, \sigma^{2}, \sigma \beta$, and $\sigma \beta$ and larger are retained. The main point to emphasize is that by the nondimensional procedure employed, some derivative product terms are of the same order of magnitude as the Boussinesq term. Third space derivatives are of higher order. These results will be explored further in the next section (PERTURBATION ANALYSIS).

\section{Boundary Conditions}

Properly posed PDE problems mean that both equations, initial conditions and boundary conditions are specified such that only one unique solution exists. The open literature contains very little information on correct initial-boundary conditions for PDE's with mixed third-order partial derivatives. The nonlinear, Boussinesq-type equations are clearly hyperbolic in that the Boussinesq term(s) only affect the finite celerity of individual wave components of the solution.

For such hyperbolic systems, the number of boundary conditions should equal the number of characteristics pointing into the region of interest (Abbott, 1966). Also, from general mathematical principles for wave type equations, (Churchill, 1962) or from practical requirements for finite-differencing the equations, it is seen that the boundary conditions must be described to an order that is one less than the highest derivative in the equations. For the long wave equations these two requirements simply mean specifying either $u$ or $h$ as a function of time at any boundary for subcritical flow (Froude number, $F_{r}<1$ ) and both $u$ and $h$ for the "upstream" boundary in supercritical flow $\left(F_{r}>1\right)$. The long wave equations only contain first derivatives.

Boussinesq-type equations contain terms that express the time rate of change of the curvature, i.e. $u_{\text {xpt }}\left(\right.$ or $\left.h_{x t t}\right)$. It would appear reasonable therefore to also require specification of the normal gradient $u$ (or $h$ ) at the boundary. In reality this simply means specification on physical grounds of one additional $u($ or $h$ ) value outside the boundary so that the first space derivative can be formulated at the boundary. Further research and study of this hypothesis is required. Unless the proper boundary 
conditions are known, it remains unlikely that an accurate numerical solution will be obtained.

\section{Practical Considerations}

The theory discussed above omits practical hydraulics considerations for turbulent boundary and internal shear stresses, flows in branches and loops, two-dimensional effects in floodplains and river bends, and others. See Cunge, Holly and Verwey (1980) for an extensive modern review of these topics and their influence on numerical methods of solution for the long wave equations.

The major factor influencing quasi-long wave theory will be the bottom shear stress approximation. Modification to the Darcy-Weisbach, Manning and Chezy type formulations (originally for steady, uniform, open channel flows) have been devised for short wave propagation in shallow, coastal waters (Kamphuis, 1975; Jonsson, 1966; Bijker, 1966, and others). These are for oscillatory-type boundary layers and progressive water waves where mean flow currents (in time) are considered negligible. Much more experimental research data is needed to determine turbulent closure coefficients for flows with strong wave-current interactions. This is the region between flows dominated by unidirectional currents (no waves) and oscillatory water waves (no currents).

Because rapidly-varied flows in rivers are dominated by mean flow currents, classic chezy type boundary resistance formulations will be incorporated in the momentum balance equations for this report. 


\section{PERTURBATION ANALYSIS}

Perturbation analysis methods are useful to study the importance of various terms in the equations of rapidly-varied flow. The complex wave propagation factor and wave celerity can be found by this technique and used to study the significance of the nonlinear, Boussinesq, bed slope and other terms appearing in the various derivations. Previous efforts in this regard for the long wave equations by Ponce and Simons (1977), and Basco (1981) should be reviewed for further details about the method.

The method is also useful to study the unstable modes of the equations and formation of roll waves. And, the complex wave propagation factor can be employed to find the response matrix used in the Filter scheme Method (FSM) of numerical integration (Basco, 1981).

\section{The Perturbed Equations}

The equations of continuity and motion are subjected to small perturbations of the form $u=u_{0}+u^{\prime} ; h=h_{0}+h^{\prime} ;$ and $\tau=\tau+\tau$ for the boundary shear stresses present in real fluid flow. Thus, uniform flow conditions $\left(u_{0}, h_{0}, \tau_{0}\right)$ are given a small perturbation ( $\left.u^{\prime}, h^{\prime}, \tau^{\prime}\right)$ and the resulting dependent variables $(u, h, \tau)$ are substituted into the basic equations. After expanding, all terms containing products of perturbations are neglected based on the key assumption that these quadratic terms are relatively small. Consequently, when applied to the Boussinesq-type equations, truly higher-order terms must also be omitted.

Long Wave Equations

The perturbed long wave equations are a convenient reference point to begin and can be written

Mass

$$
h_{t}^{\prime}+u_{0} h_{x}^{\prime}+h_{0} u_{x}^{\prime}=0
$$

Motion

$$
u_{t}^{\prime}+u_{0} u_{x}^{\prime}+g h_{x}^{\prime}+g s_{0}\left[2 \frac{u^{\prime}}{u_{0}}-\frac{h^{\prime}}{h_{0}}\right]=0
$$

for a channel of uniform bottom slope, $S_{0}$ and the friction slope, $S_{e}$ given by $\tau_{0} /$ gh (see Basco, 1981 for further details).

It is convenient to put these equations in nondimensional form. Ponce and Simons (1977) choose a set of reference dimensions for space and time that effectively omitted the bottom slope from the results. Basco (1981) extended these results to more general reference dimensions ( $\epsilon$, the space increment and $\tau$, the time increment) for ease in comparison with numerical methods based on comparable $\Delta x$ and $\Delta t$, space and time steps respectively.

The dimensionless equations of Basco (1981) are modified slightly in what follows by using the shallow water celerity, $\sqrt{g h_{0}}$ as reference velocity 
in place of the uniform flow velocity, $u_{0}$. This gave the nondimensional equations

Mass $\quad\left[c_{1} F_{0} \alpha-c_{t} \frac{\theta}{\gamma_{g}}\right] h^{*}+c_{2} \alpha u^{*}=0$

Motion $\quad\left[+\right.$ ip $\left.\frac{\gamma_{o}}{F_{0}^{2}} \alpha-k \beta\right) h^{*}+\left[i a \gamma_{g} \alpha-i \ell \frac{\theta}{F_{0}}+2 k \frac{\beta}{F_{0}}\right] u^{*}=0$

where: $\quad h^{\prime} / h_{0}=h^{*} \exp \left[i\left(\alpha x^{*}-\theta t^{*}\right)\right]$,

$u^{\prime} / \sqrt{g h_{o}}=u^{*} \exp \left[i\left(\alpha x^{*}-\theta t^{*}\right)\right]$,

$\mathrm{h}^{*} \quad=$ the dimensionless water depth,

$u^{*}=$ the dimensionless water velocity,

$x^{*}=x / \epsilon$, dimensionless distance,

$t^{*}=t / \tau$, dimensionless time,

$\alpha \quad=(2 \pi / L) \epsilon=$ dimensionless wave number,

$\theta \quad=$ dimensionless, complex radian wave frequency, i.e. the propagation factor,

$\theta_{R} \quad=(2 \pi / T) T=$ the real part of $\theta$,

$\mathrm{F}_{\mathrm{o}} \quad=$ the uniform flow Froude number, $u_{0} / \sqrt{g h_{0}}$,

$\gamma_{0} \quad=u_{0} /(\epsilon / \tau)$,

$\gamma_{\mathrm{g}} \quad=\sqrt{g \mathrm{~h}_{\mathrm{o}}} /(\epsilon / \tau)$,

$B=g s_{0} \tau / u_{0}$

and, where the following additional terms are made unity or zero to either incorporate or suppress terms in these equations,

$c_{1}=$ coefficient for $h u_{x}$ (prism-storage) and of higher order,

$c_{2}=$ coefficient for $u_{x}$ (wedge-storage),

$c_{t}=$ coefficient for time derivative (rate of rise), 
in Equation (97), and

$$
\begin{aligned}
& l=\text { local acceleration in motion equation (98), } \\
& a=\text { advection acceleration, } \\
& p=\text { pressure gradient, and } \\
& k=\text { friction-bed slope term. }{ }^{1}
\end{aligned}
$$

This change in the system of dimensionless equations over that previously defined (Basco, 1981) results in a simpler set of dimensionless paremeters $\left(F_{0}, \gamma_{g}\right.$ (or $\left.\gamma_{0}\right)$ and $\left.B\right)$ than previously defined. A thorough analysis and check was made to confirm that these equations produced identical results to those presented previously by Ponce and Simons (1977) and Basco (1981). Equations (97) and (98) are also consistent with the notation introduced by R.O. Reid (Feb., 1980, personal communication) except that the hydraulic engineer's definition of Froude number [Equation $99(j)$ ] is employed.

\section{Boussinesq-Type Equations}

The following equations shall be employed assuming constant bottom slope and real fluid flow.

Mass

$$
h_{t}+u h_{x}+h u_{x}=0
$$

Motion

$$
\begin{aligned}
& u_{t}+u u_{x}+g h_{x}+g S_{o}-g S_{e}= \\
& \underbrace{\frac{1}{3} h^{2} u_{x x t}}_{b_{1}}-\underbrace{\frac{1}{3}(u h) h_{x x t}}_{b_{2}}+\underbrace{s_{0} h u_{x t}}_{b_{s}}
\end{aligned}
$$

The perturbation analysis method is used to study the relative importance of the primary Boussinesq term $\left(b_{1}\right)$ and the two additional terms in Equation (102). As previously discussed, the second Boussinesq terms $\left(b_{2}\right)$ and the slope term ${ }^{2}(b)$ were both shown to be important as derived by variational principles and scaled against the other terms.

All derivative product terms of order $O\left(\sigma^{2}\right)$ as determined by the scaling methods of Section IITERATURE REVIEW are neglected in Equation (102). Examination of one such term will demonstrate why.

For example, Equation (90) revealed that the term $h h_{x} u_{x t}$ as derived by Venezian (1976) is of $O\left(\sigma^{2}\right)$. This term becomes in the perturbed system

\footnotetext{
IThis section uses lower case $k$ for a coefficient ( 0 or 1 ) of the friction-bed slope term and script for the wave number.

${ }^{2}$ The plus sign in this slope term comes from $-\beta=S_{0}$ for this nomenclature, and $B$ is now defined from Equation $(99 \mathrm{~m})$ and is not the bottom slope.
} 


$$
h_{0} h_{x}^{\prime} u_{x t}^{\prime}
$$

or in nondimensional terms

$$
i h_{o}^{2} \sqrt{g h_{\circ}}\left[\frac{\theta \alpha^{2}}{\epsilon^{2} \tau}\right] h^{*} u^{*}\left[\exp \left(i\left(\alpha x^{*}-\theta t^{*}\right)\right)\right]^{2}
$$

The perturbation analysis method simply becomes too mathematically intractable to handle $h^{*} u^{*}$ terms and/or terms involving products of exponentials. Put another way, Equation (103) derived from the original term $\mathrm{hh}_{\mathrm{x}} \mathrm{u}_{\mathrm{xt}}$, already neglects terms of the order $h^{\prime} h_{x}^{\prime} u_{x t}^{\prime}$ so that inclusion of such terms would be inconsistent with the perturbed long wave equations. gives

Substitution of Equation (99) into the last three terms in Equation (102)

$$
u_{t}^{\prime}+u_{o} u_{x}^{\prime}+g h_{x}^{\prime}+g s_{o}\left(2 \frac{u_{0}^{\prime}}{u_{0}}-\frac{h^{\prime}}{h_{0}}\right)=\frac{1}{3} h_{0}^{2} u_{x x t}^{\prime}-\frac{1}{3} u_{0} h_{o} h_{x x t}^{\prime}+h_{o} s_{o} u_{x t}^{\prime}
$$

as the perturbed Boussinesq equation. The left-hand-side (LHS) is identical to the long wave Equation (96) and the right-hand-side (RHS) contains the two Boussinesq terms and a slope term. It is again convenient to put Equation (105) in nondimensional form. Considering only RHS terms gives

$$
=\frac{1}{3} b_{1} h_{0}^{2}\left(\frac{i \theta \alpha^{2}}{\epsilon^{2} \tau}\right] \sqrt{g h_{o}} u^{*}-\frac{1}{3} b_{2} u_{o} h_{0}\left(\frac{i \theta \alpha^{2}}{\epsilon^{2} \tau}\right] h_{o} h^{*}+b_{s} h_{o} s_{o}\left[\frac{\theta \alpha}{\epsilon \tau}\right] \sqrt{g h_{o}} u^{*}
$$

or after multiplying through by $\tau / u_{0}$ and simplifying

$$
=\left[i \frac{b_{1}}{3}\left[\frac{\alpha_{0}^{2}}{F_{0}}\right) \theta+b_{s}\left(\frac{\gamma_{g} \alpha s_{0}^{2}}{\beta F_{0}^{2}}\right) \theta\right] u^{*}-\left[i \frac{b_{2}}{3} \alpha_{0}^{2} \theta\right] h^{*}
$$

where, in addition,

$$
\begin{aligned}
& \alpha_{0}=k h_{0}=\alpha \frac{h_{0}}{\epsilon} \\
& b_{1}=1 \text { or } 0 \text { if primary Boussinesq term is to be included, } \\
& b_{2}=1 \text { or } 0 \text { if secondary Boussinesq term is to be included, } \\
& b_{s}=1 \text { or } 0 \text { if slope Boussinesq term is to be included. }
\end{aligned}
$$

Putting Equation (106) together with the nondimensional long wave Equation (98) and summarizing gives 
Mass $\quad\left[c_{1} F_{0} \alpha-c_{t} \frac{\theta}{\gamma_{g}}\right] h^{*}+c_{2} \alpha u^{*}=0$

Motion $\left[\right.$ ip $\left.\frac{\gamma_{0}}{F_{0}^{2}} \alpha+i \frac{b_{2}}{3} \alpha_{0}^{2} \theta-k \beta\right] h^{*}+$

$$
\left[i a \gamma_{g} \alpha-i \ell \frac{\theta}{F_{0}}-i \frac{b_{1}}{3}\left[\frac{\alpha_{0}^{2}}{F_{0}}\right] \theta-b_{s}\left[\frac{\gamma_{g} \alpha S_{\alpha}^{2}}{\beta F_{0}^{2}}\right] \theta+2 k-\frac{\beta}{F_{0}}\right] u^{*}=0
$$

Equations (97) and (108) form the nondimensional, Boussinesq equations to be employed for all subsequent analysis. They contain eleven terms and ten separate coefficients which can be either 1 or 0 to study special cases.

\section{Simplified Flow Cases}

A check of the simplified cases gives confidence in the complete equations. Note that the true, physical, dimensionless wave celerity is found from $\theta_{R} / \alpha=\gamma$ and is identical to the courant number.

\section{Pure Advection}

In the motion equation, taking $a=l=1$ and $p=k=b_{1}=b_{2}=b_{s}=0$ gives simply

$$
\theta=\gamma_{0} \alpha
$$

or in dimensional units, the wave celerity, $\omega / k$ becomes

$$
\omega / k=u_{0}
$$

where $\omega$ is the radian wave frequency $(2 \pi / T)$ and $k$ is the wave number $(2 \pi / L)$. The celerity of disturbance propagation for this case is simply $u_{0}$ for pure advection.

\section{Lagrange Celerity}

More realistically, the celerity of a shallow water, long wave distrubance (the Lagrange celerity) can be found when the pressure gradient term is included. Taking $a=l=p=1$ and $k=b_{1}=b_{2}=b_{s}=0$ and all continuity terms $\left(c_{1}=c_{2}=c_{t}=1\right)$ gives, solving $(97)^{2}$ and ${ }^{3}(108)$ together,

$$
\theta=\gamma_{0} \alpha \pm \frac{\gamma_{0} \alpha}{F_{0}}
$$


or in dimensional terms

$$
\omega / k=u_{0} \pm \sqrt{g h_{0}},
$$

as expected.

In both of these cases taking $k=0$ gave only real roots to $\theta$.

Long Waves with Friction

In this case, all terms are unity except $b_{1}=b_{2}=b_{8}=0$. This is the long wave case considered in Equations (97) and (98) and the resulting expression for $\theta$ becomes

$$
\theta=\gamma_{0} \alpha-i \beta \pm \sqrt{\gamma_{g}^{2} \alpha^{2}-\beta^{2}+i \beta \gamma_{0} \alpha}
$$

It was found to reduce to the expression given by Ponce and Simons (1977, Eqn. $36, p .1466)$ when $\epsilon=h_{0} / s_{0}$ and $\tau=h_{0} /\left(u_{0} s_{0}\right)$ were used as reference dimensions. Equation (113) also reduces to that obtained by Basco (1981) with $\epsilon=h_{0} ; \tau=h_{\alpha} / u_{o}$ and using $u^{\prime} / u_{0}=u^{*} \exp \left[i\left(\alpha x^{*}-\theta t^{*}\right)\right]$ as the nondimensional perturbed velocity.

\section{Diffusion and Kinematic Wave Models}

In classical hydraulics, when the acceleration of long waves $\left(b_{1}=b_{2}=\right.$ $\left.b_{s}=0\right)$ is considered negligible $(l=a=0)$, but friction and pressure gradient are in balance $(p=k=1)$

then

$$
\theta=\frac{3 \gamma_{\alpha} \alpha-i\left(\gamma_{\alpha}^{2} / \beta\right) \alpha^{2}}{2}
$$

Taking only the real part of $\theta$ and putting in dimensional units gives

$$
\frac{\omega}{k}=\frac{3}{2} u_{0}
$$

which checks with the accepted speed of travel theorized for such waves in the literature (see, e.g., Stoker, 1957). This special case has come to be called a diffusion wave model in the literature.

Also taking $p=0$ gives the same results because $p$ produced the imaginary part of Equation (114). This case is called a classic kinematic wave model since only the friction-slope terms control the motion. 


\section{Linearized Boussinesq Equation}

In classical wave theory, linearization of these equations means neglecting the advection term $(a=0)$ and also the uh term in the continuity equation $\left(c_{1}=0\right)$. For a horizontal bottom with no friction and only considering the primary Boussinesq term $\left(k=b_{0}=b_{2}=0\right)$ leaves $\left(c_{t}=c_{2}=1\right.$; $l=p=b_{1}=1$ ). Solving for the characteristic equation and then $\theta$ gives

$$
\theta=\left[ \pm \frac{\gamma_{0} \alpha}{F_{0}}\right]\left[\frac{1}{\left(1+\alpha_{0}^{2} / 3\right)^{1 / 2}}\right]
$$

or in dimensional terms

$$
\frac{\omega}{k}= \pm \frac{\sqrt{g h_{0}}}{\left(1+\frac{1}{3} k^{2} h_{0}^{2}\right]^{1 / 2}}=\frac{\sqrt{g h_{0}}}{\left(1+\frac{4 \pi^{2}}{3} \frac{h_{0}^{2}}{L^{2}}\right]^{1 / 2}}
$$

Equation (117) checks with the literature (Whitham, 1974, Eqn. 13.93, p. 462 ). Whitham (1974) also shows how this linearized dispersion relation for small $\left(k h_{0}\right)^{2}$ agrees with the classic dispersion equation $\left(\omega^{2}=g k\right.$ tanhkh $)$ from small amplitude, linear, (Airy) wave theory to the first approximation beyond long wave theory $\left(i . e . \omega / \hbar=\sqrt{g h_{0}}\right)$.

Examination of Equation (117) now reveals how the Boussinesq term causes frequency dispersion as described in Section 1 and Equation (3) taking $d=h_{0}$. The $(1 / 3) k^{2} h_{0}^{2}$ term in the denominator increases for shorter wave lengths creating slower moving waves than the very long waves $\left(k^{2} h{ }^{2} \rightarrow 0\right)$. Linearization of the equations is equivalent to cases with very small Froude number flows with small disturbances since $\epsilon \approx \sigma^{2}$ and

$$
\sigma^{2}=\frac{1}{(2 \pi)^{2}} k^{2} h_{0}^{2}
$$

This shall be verified with some numerical examples later in this section.

\section{Classic Boussinesq Equation}

The advection term and all continuity terms are now retained $\left(c_{1}=c_{2}=\right.$ $c_{f}=1 ; 1=a=p=b_{1}=1$ ) and the case for a horizontal bed with no friction $(k=0)$ and no other Boussinesq terms $\left(b_{2}=b_{8}=0\right)$ is reexamined. The expression for $\theta$ becomes

$$
\theta=\gamma_{0} \alpha\left\{1 \pm\left[1+\frac{\left(1-F_{0}^{4}\right)}{\left(1+\frac{\alpha_{0}^{2}}{3}\right) F_{0}^{2}}\right]^{1 / 2}\right\}
$$

For very long waves, $\alpha_{0}^{3} / 3 \rightarrow 0$ and Equation (119) reduces to Equation (111) for the Lagrange celerity for all Froude numbers. For linearized conditions, $F_{0}{ }^{2} \rightarrow 0$ and Equation (119) reduces to the linearized Boussinesq celerity, Equation (116), as expected. 
The celerities given by Equation (119) should be compared with those given by first order cnoidal wave theory (See e.g., Svendsen, 1974) since cnoidal waves are a solution of the Boussinesq (or KdV) equation (Whitham, 1974). This is the first case where the dispersion relation between frequency $\omega$ and wave number $k$ involves the wave amplitude which is here associated with the Froude number, $F_{0}$ in Equation (119). The influence of $F_{0}$ shall be examined with numerical examples below.

The limit of cnoidal wave theory as the dispersed wave length becomes infinitely long is the solitary wave theory (i.e. for a single hump of constant shape moving with constant speed). The key aspect of which is the fact that theoretically, the peak height, $\epsilon_{\max }=0.78$ and experimentally this has been confirmed to be about $\epsilon_{\max } \approx 0.7$. Thus, the Boussinesq equations are applicable for engineering purposes far beyond the theoretical limits for small $\epsilon$ and $\sigma^{2}$ values. See Whitham (1974, p. 467-471) for further discussion.

Boussinesq Theory on Plane Slope with Friction

The full equations (97) and (108) can be written in matrix notation as

$$
\left[\begin{array}{cc}
\left(c_{1} F_{0} \alpha-c_{t} \frac{\theta}{\gamma_{g}}\right) & \left(c_{2} \alpha\right) \\
\left(i p \frac{\gamma_{0}}{F_{0}^{2}} \alpha+i \frac{b_{3}}{3} \alpha_{0}^{2} \theta-k \beta\right) & {\left[i \gamma_{g} \alpha-i \ell \frac{\theta}{F_{0}}-i \frac{b_{1}}{3}\left(\frac{\alpha_{0}^{2}}{F_{0}^{2}}\right) \theta-b_{s}\left(\frac{\gamma_{g} \alpha S_{0}^{2}}{\beta F_{0}^{2}}\right) \theta+2 k \frac{\beta}{F_{0}}\right]}
\end{array}\right]\left[\mathrm{h}^{*}\right]=0
$$

to give, after simplification and collection of terms, the characteristic equation

$$
\begin{aligned}
& \left.\frac{1}{\gamma_{0}}\left[c_{t} l+\frac{c_{t} b_{1}}{3} \alpha_{0}^{2}\right)-i\left(c_{t} b_{s}\right) \frac{\alpha S_{0}^{2}}{\beta F_{0}^{2}}\right] \theta^{2} \\
& -\left[\frac{1}{3}\left(c_{1} b_{1}+c_{2} b_{2}\right) \alpha_{0}^{2} \alpha+\left(c_{1} l+c_{t} a\right) \alpha-i\left[\frac{2 c_{t} k \beta^{2} F_{0}+c_{1} b_{s} \gamma_{g} \gamma_{0} s_{0}^{2} \alpha^{2}}{\gamma_{0} \beta F_{0}}\right)\right] \theta \\
& +\left[\left(c_{1} a-\frac{c_{2} p}{F_{0}^{2}}\right) \gamma_{0} \alpha^{2}-i\left(c_{2} k+2 c_{1} k\right) \beta \alpha\right]=0
\end{aligned}
$$

Careful backchecking proves that Equation (121) reduces to all previously discussed cases when the appropriate terms are eliminated. Equation (121) is in quadratic form of $\theta$ and a general computer program was developed using complex arithmetic for its solution. The main purpose was to compute the celerity over a wide range of dimensionless wave numbers, $\alpha(0<\alpha \leqslant \pi)$ and study friction-slope effects, nonlinearity effects, and the additional Boussinesq terms included by the $b_{2}$ and $b_{s}$ coefficients.

A total of 37 tests were conducted as summarized in Table 3 . These covered a wide range of bed slopes and Froude numbers over the practical limits of boundary roughness and beyond. Figure 2 gives an indication of the 
Table 3 Celerity tests

\begin{tabular}{|c|c|c|c|c|c|c|c|}
\hline $\begin{array}{c}\text { Bed } \\
\text { Slope } S_{0}\end{array}$ & $\begin{array}{l}\text { Test } \\
\text { No. }\end{array}$ & $\begin{array}{c}\text { Water } \\
\text { Depth } h_{0}\end{array}$ & $\begin{array}{c}\text { Water } \\
\text { Velocity } u_{0}\end{array}$ & $\begin{array}{l}\text { Froude } \\
\text { No, } F_{0}\end{array}$ & $\begin{array}{c}\text { Chezy } \\
C_{C}\end{array}$ & $\underset{n}{\text { Manning }}$ & Remark \\
\hline 0.0001 & $\begin{array}{r}17 \\
28 \\
1 \\
29 \\
18 \\
30 \\
19 \\
31 \\
20\end{array}$ & $\begin{array}{r}20.0 \\
20.0 \\
20.0 \\
14.0 \\
12.0 \\
10.0 \\
10.0 \\
4.0 \\
2.0\end{array}$ & $\begin{array}{l}0.1 \\
0.5 \\
1.0 \\
1.5 \\
2.0 \\
3.5 \\
7.0 \\
7.0 \\
7.0\end{array}$ & $\begin{array}{l}0.00394 \\
0.0197 \\
0.0394 \\
0.0707 \\
0.102 \\
0.195 \\
0.39 \\
0.62 \\
0.87\end{array}$ & $\begin{array}{l}2.2 \\
11 \\
22.4 \\
40 \\
58 \\
110 \\
221 \\
350 \\
495\end{array}$ & $\begin{array}{l}1.12 \\
0.217 \\
0.110 \\
0.058 \\
0.039 \\
0.020 \\
0.010 \\
0.005 \\
0.0034\end{array}$ & $\begin{array}{l}\text { Too rough! } \\
\text { Too smooth! } \\
\text { Too smooth! }\end{array}$ \\
\hline 0.001 & $\begin{array}{r}5 \\
8 \\
32 \\
2 \\
33 \\
3 \\
34 \\
4 \\
6 \\
16\end{array}$ & $\begin{array}{l}20.0 \\
20.0 \\
14.0 \\
12.0 \\
10.0 \\
10.0 \\
4.0 \\
2.0 \\
1.0 \\
1.0\end{array}$ & $\begin{array}{l}0.1 \\
1.0 \\
1.5 \\
2.0 \\
3.5 \\
7.0 \\
7.0 \\
7.0 \\
8.0 \\
11.344\end{array}$ & $\begin{array}{l}0.039 \\
0.0394 \\
0.0707 \\
0.102 \\
0.195 \\
0.390 \\
0.617 \\
0.873 \\
1.41 \\
2.0\end{array}$ & $\begin{array}{c}0.71 \\
7.1 \\
12 . \\
18 . \\
35 . \\
70 . \\
111 . \\
156 . \\
253 . \\
359 .\end{array}$ & $\begin{array}{l}3.46 \\
0.347 \\
0.182 \\
0.123 \\
0.062 \\
0.031 \\
0.017 \\
0.011 \\
0.006 \\
0.004\end{array}$ & $\begin{array}{l}\text { Too rough. } \\
\text { Too rough. } \\
\text { Too smooth. } \\
\text { Too smooth. }\end{array}$ \\
\hline 0.01 & $\begin{array}{r}7 \\
14 \\
35 \\
13 \\
36 \\
12 \\
9 \\
10 \\
11 \\
15\end{array}$ & $\begin{array}{r}20.0 \\
12.0 \\
10.0 \\
10.0 \\
4.0 \\
2.0 \\
1.0 \\
1.0 \\
3.0 \\
0.5\end{array}$ & $\begin{array}{l}1.0 \\
2.0 \\
3.5 \\
7.0 \\
7.0 \\
7.0 \\
8.0 \\
11.344 \\
40.0 \\
40.0\end{array}$ & $\begin{array}{l}0.0394 \\
0.102 \\
0.195 \\
0.390 \\
0.617 \\
0.873 \\
1.41 \\
2.0 \\
4.07 \\
9.97\end{array}$ & $\begin{array}{l}2.2 \\
5.8 \\
11 . \\
22 . \\
35 . \\
49.5 \\
80 . \\
113 . \\
231 . \\
565 .\end{array}$ & $\begin{array}{l}1.10 \\
0.390 \\
0.20 \\
0.0988 \\
0.054 \\
0.0338 \\
0.019 \\
0.013 \\
0.0077 \\
0.0023\end{array}$ & $\begin{array}{l}\text { Too smooth. } \\
\text { Too smooth. }\end{array}$ \\
\hline 0.1 & $\begin{array}{l}21 \\
22 \\
37 \\
23 \\
24 \\
25 \\
26 \\
27\end{array}$ & $\begin{array}{r}12.0 \\
10.0 \\
4.0 \\
2.0 \\
1.0 \\
1.0 \\
3.0 \\
0.5\end{array}$ & $\begin{array}{l}2.0 \\
7.0 \\
7.0 \\
7.0 \\
8.0 \\
11.344 \\
40 . \\
40 .\end{array}$ & $\begin{array}{l}0.102 \\
0.390 \\
0.617 \\
0.873 \\
1.41 \\
2.00 \\
4.07 \\
9.97\end{array}$ & $\begin{array}{c}1.8 \\
7.0 \\
11 . \\
16 \\
25 \\
36 \\
73 \\
179\end{array}$ & $\begin{array}{l}1.23 \\
0.312 \\
0.171 \\
0.106 \\
0.0589 \\
0.0415 \\
0.0245 \\
0.0074\end{array}$ & Too smooth. \\
\hline
\end{tabular}


Froude No. $\left(F_{0}\right)$

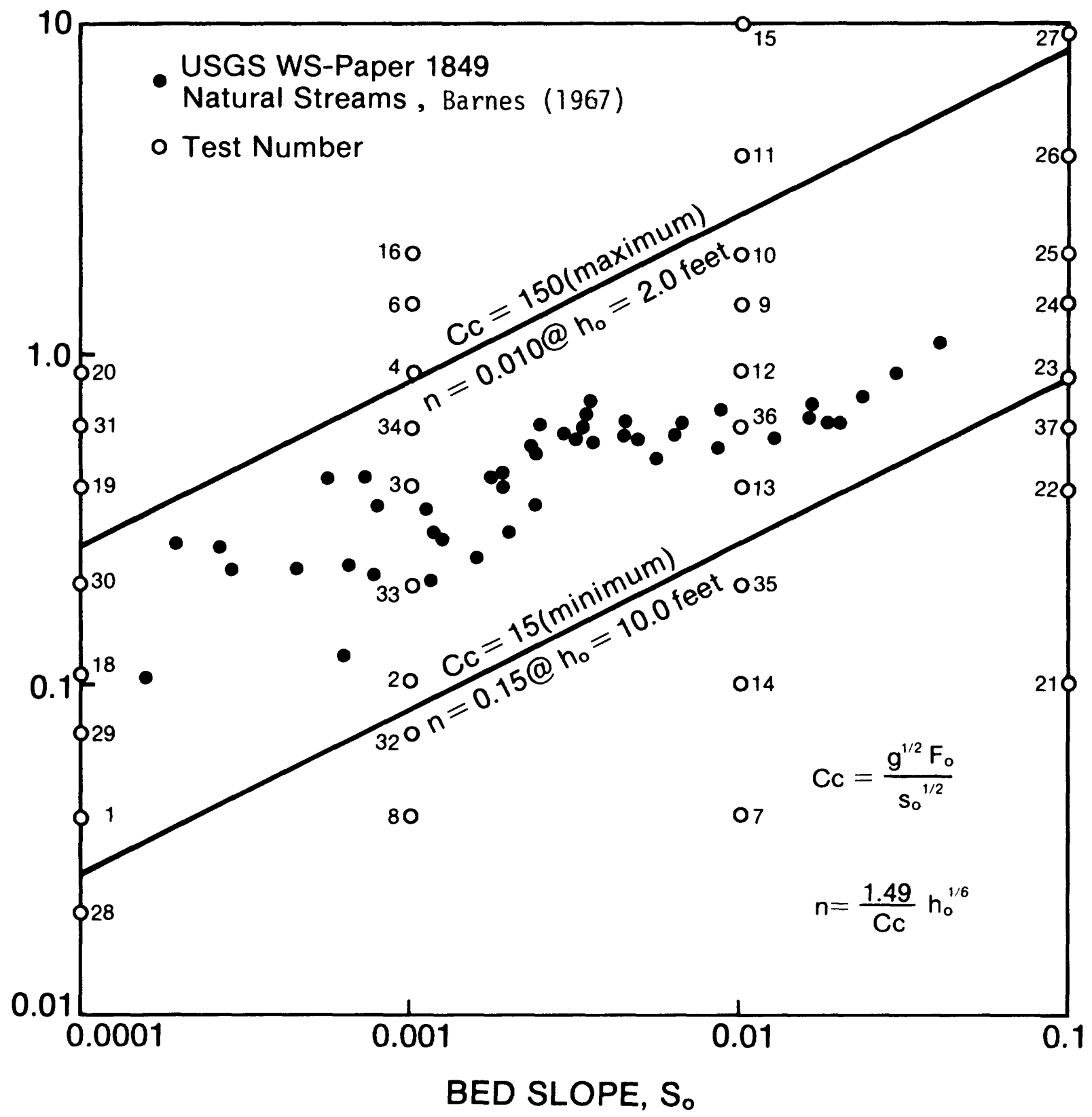

Figure 2. Range of Flow Conditions Tested 
spread of test coverage compared with results from real, natural streams as measured by Barnes (1967). An example of the test results (Test no. 2) is presented as Table 4. It lists values of the dimensionless wave celerity, $\gamma_{+}$for the positive characteristic, for 8 representative ${ }^{*}$ wave numbers for each of 15 different test cases. Each test case gives a different combination of terms taken in the continuity and motion equations. Results can best be summarized for each "case" tested.

\section{Summary of Cases Tested}

Case 2 (pure advection), Case 3 (Lagrange celerity) and Case 6 (diffusion wave) all gave results as expected. Since the reference dimensions taken were

$$
\begin{aligned}
\epsilon & =\mathrm{h}_{0} \\
\tau & =\mathrm{h}_{\mathrm{o}} / \mathrm{u}_{0}
\end{aligned}
$$

then $\epsilon / \tau=u_{0}$ became the reference celerity. Values of $\gamma_{+}$therefore simply indicate the ratio of true celerity to $u_{0} \cdot$ For pure advection (Case 2 ) this was always unity and equalled 1.5 for the diffusion wave (Case 6) as given by Equation (115). For very large slopes and Froude numbers, the Lagrange celerity (Case 3) approached the uniform flow velocity, u so that $\gamma_{+}$ approached unity. Note also in Table 4 that $\gamma_{+}$values for the Lagrange celerity are constant for all wave numbers (lengths). Case 5 attempted to test the theory with zero velocity (still water). This produced $F=0$ and a singularity in the equations that requires limit analysis for solution. Results for case 5 could not be obtained from the computer code for Equation (121) and were consequently delayed for future study.

\section{Case 4. Linearized Boussinesg}

For engineering purposes, it is desired to learn when the full nonlinear Boussinesq equations are required. This was accomplished by defining the percent celerity difference for any $\alpha$ as

$$
\text { Difference }=\frac{\gamma_{\mathrm{B}}-\gamma_{\mathrm{LB}}}{\gamma_{\mathrm{B}}} \cdot 100
$$

where the subscript $B$ stands for full Boussinesq and LB, the linearized Boussinesq equations, respectively. The results for $\bar{a}$ ll $F_{0}$ are presented in Figure 3 and show that for $5 \%$ accuracy or better, when $F_{0}>0.05$ the full Boussinesq equations must be employed. For computation purposes, it is best and perhaps algorithmically more tractable to always use the full equation for any Froude number. Figure 4 gives an example of the difference for all $\alpha$ when $\mathrm{F}_{0}=0.102$ on a 0.001 slope (Test no. 2 ).

Case 7. Full long wave theory

As expected, only the very long wave components $(\alpha \rightarrow 0)$ are influenced by the empirical, boundary resistance law of the Chezy or Manning type. When

*Actually 30 values for $0<\alpha \leqslant \pi$ computed but only 8 tabulated for comparison
purposes. 


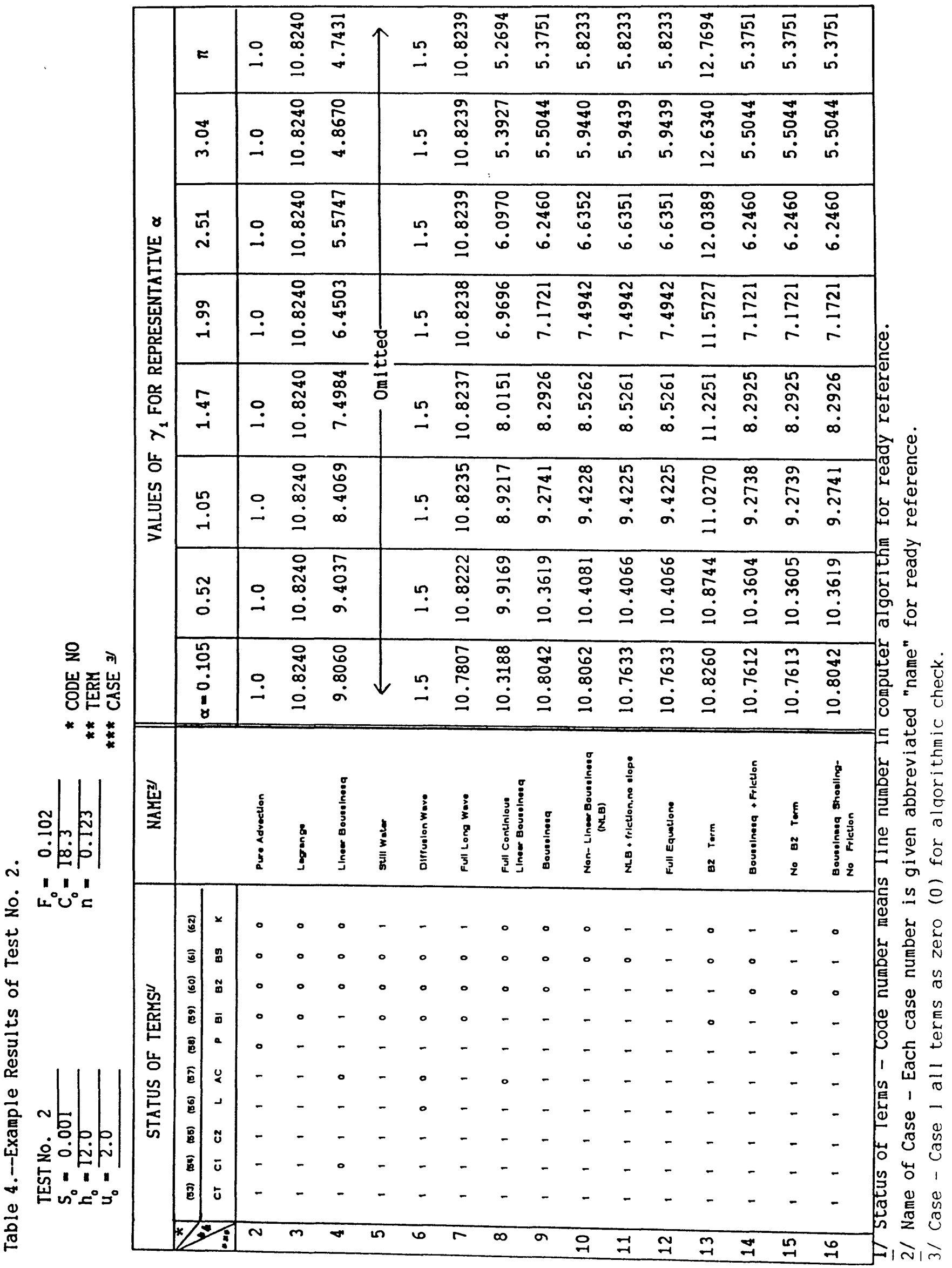




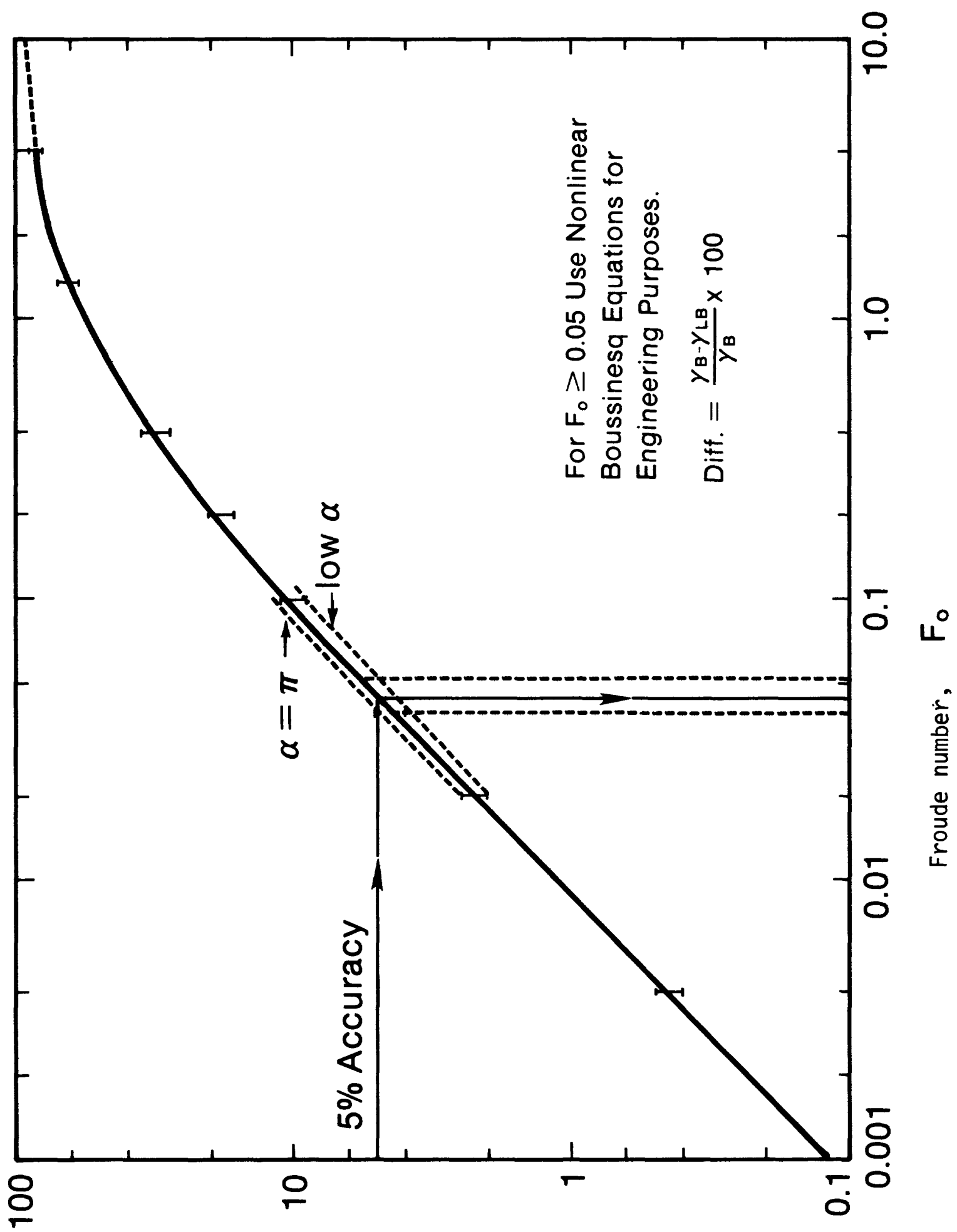

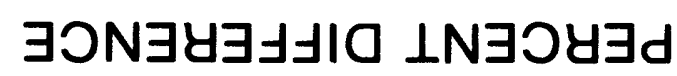

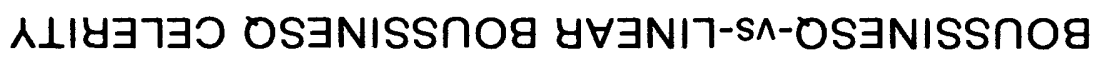




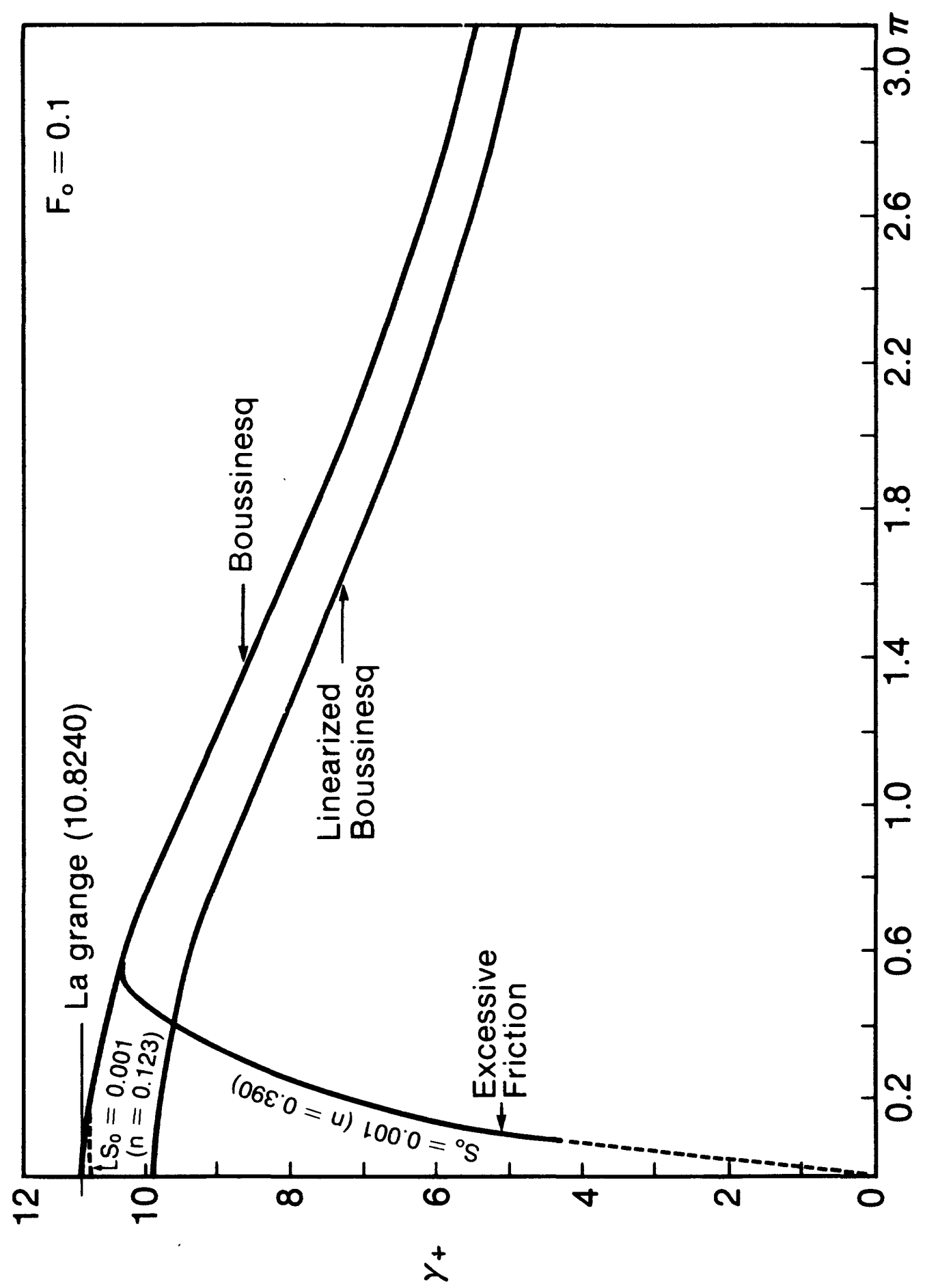

ธุํำ

8

ปิ

윽으

음

尔

$4 \stackrel{0}{0}$

$5 \bar{n}$

음

ช $\quad$ 픈

5-

$\frac{1}{0}$
$\frac{1}{0}$
$\frac{1}{0}$
0
$\frac{0}{3}$

Dे
$+\pi$

$>$ 무

$\pm N$

ธิธ ช

巡

$\sum_{0}^{0} \frac{1}{1}$

용

藏

ธธ㇒士

ज०

ญ्ट

兑

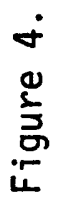

ıәqunu ә^ем 
friction values are excessively high, say $n=0.390$, (Test no. 14, $\mathrm{S}_{0}=0.01$ ) then the celerity is excessively damped for long wave components (see Fig. 4). More realistic celerity reductions by friction are also shown in Figure 4 and only affect the long wave celerities. Short wave components return to the Lagrange celerity, i.e. for frictionless flows. The latter is also true for all wave length components when frictional effects are very small, as expected.

Where $F_{0}=2.0$, the dimensionless celerity for the full Saint-Venant equations is 1.5000 and identical to the Lagrange celerity for all slopes. This is the critical, Jefferys-Vedernikov stability criterion $\left(F_{0}=2.0\right)$ for formation of roll waves (Chow, 1959; Henderson, 1966; Abbott, 1979). For $F<2$ the celerity of long waves is less than the Lagrange celerity. For $\mathrm{F}^{\circ}>2$ it is greater than the Lagrange celerity but approaches it as $\alpha \rightarrow \pi$. case 8 did not prove important.

\section{Case 9. Boussinesq Equations}

It was found that for long waves as $\alpha \rightarrow 0$, the Boussinesq celerity was always less than the Lagrange celerity. The celerity decreased as $\alpha \rightarrow \pi$, as expected, and was roughly one-half as much at $\alpha=\pi$ than it was at $\alpha=0$, for all slopes when $F_{0}<1$ (See Figure 4). Clearly, the influence of the Boussinesq term $\left(b_{1}\right)$ on wave celerity is significant and cannot be neglected for rapidly-varied, shortwave flows. For $F_{0}>1$ and as $F_{0}$ increased further, the celerity for all wave numbers approaches unity (i.e., $u_{0}$ ), as expected. Consequently Boussinesq celerity changes are even more important for shorter wave computations on flatter slopes with low Froude numbers.

Cases $10,11,12$, and 13. The $\mathrm{b}_{2}$ Term

These cases incorporated the secondary Boussinesq term $\left(b_{2}\right)$ Equation (91) in various combinations with other terms including $b_{1}$, and all by itself with no $b_{1}$ term (Case 13). In all tests, the long wave celerity with the $b_{2}$ term was greater than the Boussinesq celerity (Case 9). In fact, for those few tests when Case 13 was included, the wave celerity was greater than the Lagrange celerity when $F_{0}>1.0$ for all wave lengths. Thus inclusion of the $\mathrm{b}_{2}$ term means adding a correction to increase the Boussinesq celerity for all wave numbers $0<\alpha \leq \pi$. Since the $b_{2}$ term behaves in this fashion, it undoubtedly requires additional higher-order-terms and is itself a higher order term. For this reason we must conclude that the $b_{2}$ term is incorrect to include alone as another or secondary Boussinesq term. Since the results with the $b_{2}$ term do not reduce to the Lagrange celerity when and where expected, we shall omit the $\mathrm{b}_{2}$ term from further study except in conjunction with other, higher-order terms.

Case 14. Boussinesq Equation with Friction

When comparing Case 14 results with friction, with those of Case 9 (no friction) it was found that only the very long wave components $(\alpha \rightarrow 0)$ are damped. On steeper slopes, as $F_{0}$ increases, shorter wave length components do become damped but never the very short components $(\alpha>1)$. In effect, these shorter wave components, with smaller orbits never "feel" bottom. However, it 
seems physically reasonable that the Chezy or Mannings empirical resistance formulas, developed for uniform, open channel flows, are inadequate for Boussinesq type flows. Further research is needed here into the use of those modified, wave-resistance equations developed for short-wave, coastal hydrodynamics (e.g., Kamphuis, 1975; Jonsson, 1966; and Bijker, 1966).

Cases 15 and 16. The slope-Boussinesg Term $\left(b_{s}\right)$

Finally, the inclusion of the additional term $\left(b_{s}\right)$ in the motion equation arising on constant sloped boundaries (see, e.g. Table 1 ) has been found to produce very minor changes all tests conducted. Its influence is clearly of higher-order significance.

\section{Conclusions from Perturbation Analysis}

Based upon the perturbed equations employed for this analysis (Equations 97 and 108) and the resulting complex wave propagation factors and wave celerities for a wide range of practical flow conditions, we can draw the following conclusions:

1. All results confirm existing, simplified analytic solutions which gives confidence to the new results.

2. The $b_{2}$ term is an incorrect addition to the Boussinesq equations by itself. It is possible (hypothesis) that other higher-order terms must be included in its use.

3. The $b_{g}$ term is small and safely neglected for the order of the Boussinesq theory.

4. For engineering purposes use the full Boussinesq equations for $F_{0} \geqslant 0.05$.

5. For rapidly-varied flows, neglect of the Boussinesq term $\left(b_{1}\right)$ produces over $100 \%$ error in wave celerity for short wave components. Use of the full Boussinesq equations means waves automatically reduce to long wave celerities for long wave components for all Froude conditions.

6. As expected, for large $F_{0}$, celerity approaches $u_{0}$ for pure advection.

7. Chezy or Manning type resistance formulas should be replaced by a more general equation to include some frictional damping of short wave celerities.

The results of all 37 tests are summarized and presented in the Appendix. 
Based upon a given set of scaling parameters, a dimensionless equation of motion was derived from momentum conservation that produced a second Boussinesq term $\left(b_{2}\right)$ of $O\left(\sigma^{2}\right)$ from a derivative product. The perturbation analysis conducted in this chapter proved that this additional term significantly increases the celerity for all $\alpha$ over that found for the "Boussinesq" equations with only a "b for the short wave components $(\alpha \rightarrow \pi)$ and larger Froude numbers. A clear inconsistency arises with the $b_{2}$ term when $F_{r}>1.0$. Here the celerity is greater than the long wave celerity which is clearly incorrect. For this reason, we must conclude that the scaling methods employed above require restudy and that the $b_{2}$ term must be considered with all other secondary terms and not by itself.

Consequently, the Boussinesq equations to be employed for numerical integration as a result of this study are

Mass :

$$
h_{t}+u h_{x}+h u_{x}=0
$$

Motion:

$$
u_{t}+u u_{x}+g h_{x}+g\left(s_{0}-s_{e}\right)=\frac{1}{3} h^{2} u_{x x t}+s_{0} h u_{x t}
$$


For unsteady, one-dimensional propagation problems, the method-ofcharacteristics and finite-difference mathods are commonly employed to numerically integrate the long wave equations. Basco (1981) and Reid and Basco (1982) recently described a numerical convolution procedure, hereafter called the filter-scheme method which will also be discussed. Only the basic ideas, advantages, and/or limitations of these three methods as applied to the Boussinesq equations are considered for this report. Treatment of the mixed-derivative term to insure convergence and accuracy is of primary concern. The greatest emphasis is on the finite-difference method since it is most pracrical for irregular topography.

\section{Method of Characteristics}

Because the method of characteristics is more cumbersome to program on the computer, it has generally been surplanted by difference techniques. Its greatest strength in recent years is for treating radiating waves at boundaries so that incoming boundary conditions are not affected. The method requires knowledge of wave celerities to trace the characteristics plus the local quasi-invariants. This information can be obtained from the complex wave propagation factor, $\theta$ as described in the previous section.

Madsen and Mei (1969) and Madsen, Mei and Savage (1970) describe the solution methods and results for solitary and short period waves transforming over sloped bottoms. The method of characteristics was employed and the characteristic equations solved numerically using difference methods and iterative techniques. A comparison with results from large scale physical experiments is shown in Figure 5, in which the agreement between experiments and the Boussinesq theory is quite good. The wave shoaling height (crest to trough) is said to follow that of cnoidal wave theory and deviates substantially from classical, linear (Airy) wave theory shoaling.

No other solutions of the Boussinesq equations in their characteristic form are known to the writer.

\section{Finite-Difference Methods}

Present Methods

The first application of the finite-difference method to the Eulerian form of the equations was by Peregrine $(1966,1967)$. Simple, first-order time derivatives were employed and centered, space derivatives with local values taken as averages over two time levels. This required iterative procedures using the continuity equation to obtain first estimates for water levels at the upper time level for use in the Boussinesq equation. The mixed derivative, Boussinesq term was approximated as

$$
u_{x x t} \cong \frac{\left(u_{x x}\right)_{j}^{n+1}-\left(u_{x x}\right)_{j}^{n}}{\Delta t}
$$

with centered, second differences used for $u_{x x}$. No effort was made to remove the truncation errors from the other differenced terms nor was their influence addressed in this early work. 


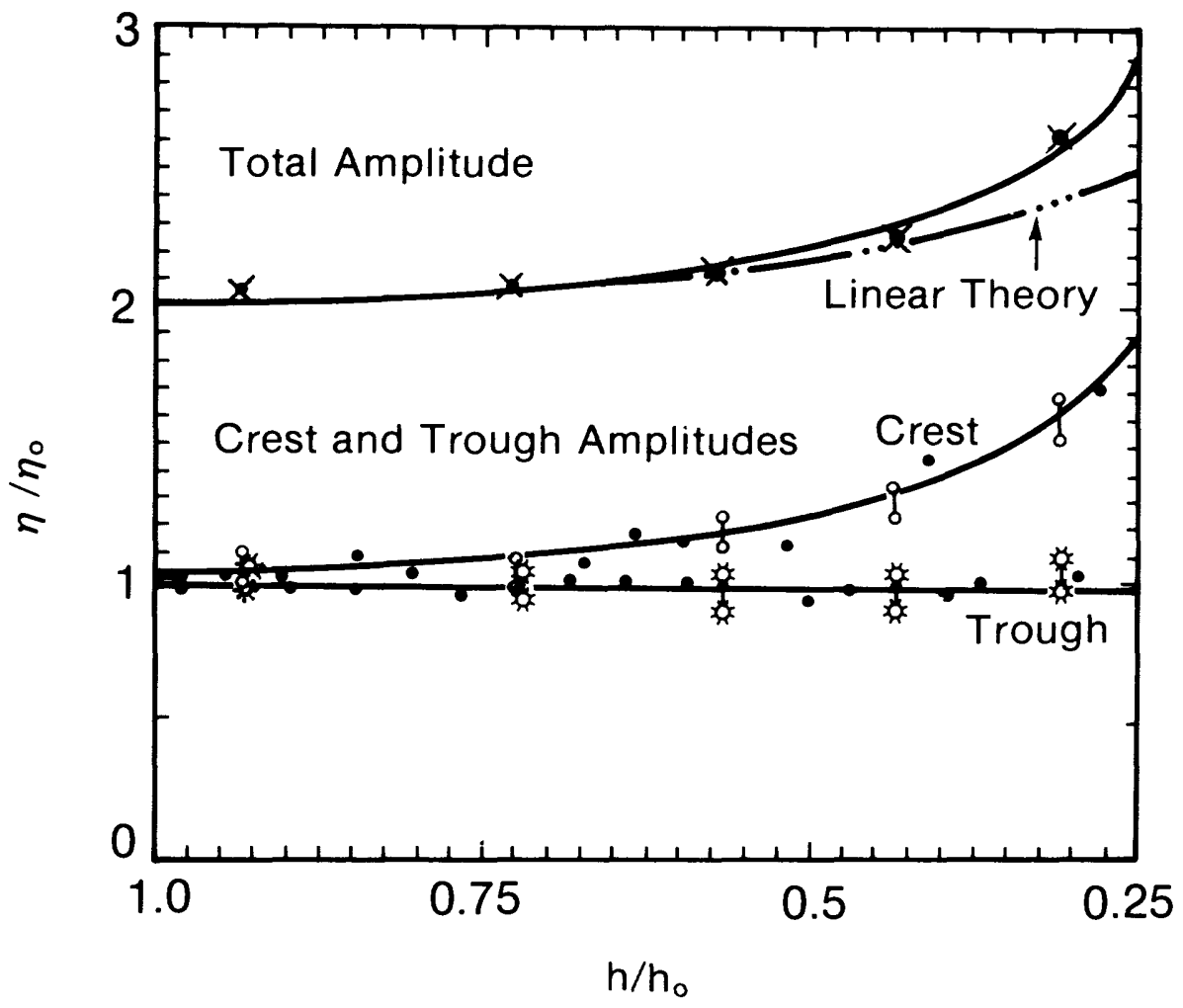

Fig. 5--Amplitude variation of a periodic wave during shoaling. Experiment: - - crest; trough; averaged total amplitude from crest to

through; - present theory. (From Madsen, Mei, and Savage, 1970)

To see the importance of the truncation error, consider a simple forward time derivative for $u_{t}$

$$
u_{t}=\frac{u_{j}^{n+1}-u_{j}^{n}}{\Delta t}-\left[u_{t t} \frac{\Delta t}{2 !}+u_{t t t} \frac{\Delta t^{2}}{3 !}+\text { H.O.T. }\right]
$$

where the bracketed terms are the truncation errors as found by a formal Taylor's series expansion. From the linearized, long wave equations for continuity and motion.

$$
\begin{aligned}
& u_{t t} \approx g h u_{x x}=c^{2} u_{x x}, \quad c=\sqrt{g h} \\
& u_{t t t} \approx c^{2} u_{x x t}
\end{aligned}
$$

Clearly the first two terms from the truncation error for $u_{t}$ in Equation (125) are terms as equally important as the Boussinesq term. The second term even has the same form, i.e. $\frac{c^{2} \Delta t^{2}}{6} u_{x x t}$. To numerically integrate using first-order generates truncation error terms that may be larger and dominate the physical Boussinesq term under investigation. Gross inaccuracies and grid-size dependent results are created that may (or may not) be physically realistic. 
Small variations in the momentum flux incident upon a short wave system can have a very great effect upon the celerities, wave lengths and wave amplitudes (Lighthill and Whitham, 1955). The variations in momentum flux generated by the truncation errors of the long wave terms in the horizontal momentum balance will be extremely significant when short waves are present which are represented by mixed third derivatives. As noted by Abbott (1979)

\begin{abstract}
"Considerable effort must then be expended to reduce the truncation error generated by the difference approximations to the nearly horizontal flow equations if unacceptable errors are to be avoided in short-wave modeling." (p. 216-217)
\end{abstract}

Numerical modeling efforts in the early 1970's at Stanford University are discussed by street, Chan and Fromm (1970) and in later years Roberts and street (197.5) and street (1976). Their "APPSIM" code based on the equations of Peregrine (1967) uses an iterative, implicit predictor-corrector method with only the long wave equations employed in the predictor step. Although iterations are carried out within each time step to a high degree of convergence, the overall accuracy of the numerical analog equations remains questionable because truncation error terms were neglected during the corrector step when the Boussinesq term was included. No one-dimensional wave shoaling experiments were made for comparison with physical measurements as in Figure 5. A comparison of two-dimensional wave transformation by a circular submarine seamount with some laboratory scale experiments proved inconclusive. This was said to be due to the computer core storage allotment being too small resulting in a very coarse grid (1-2 grid divisions) for the short wave components. Even so, the computed properties of the waves were said to be "... smooth and well behaved" (Street, Chan, and Fromm, 1970, p.21). In retrospect, this may have been caused by the excessive truncation error present in the scheme causing numerical dissipation. It is unfortunate that more emphasis was not put on calibration and verification accuracy of the scheme.

Hebenstreit and Reid (1978) employed the two-dimensional stanford code (APPSIM2) to study solitary wave motion reflecting from vertical barriers and refracting over gently sloped bathymetry. It is worth repeating that the system of equations in Eulerian form as proposed by Peregrine (1967) were employed, i.e., Equations (20) and (21). These researchers verified that the numerical scheme is stable (using linear stability analysis methods) with only one iteration of the second or corrector step. However, a second iteration of the corrector step within each time step tended to smooth out high frequency computational oscillations during long simulations. The scheme is fully dense in that values of $u, v, n$, and $h$ are required at all grid points for the computation.*

The numerical accuracy of the stanford algorithm was never explicitly addressed. In fact, their conclusions were only concerned with the physical mechanisms inherent in the reflection and refraction of solitary waves. Thus it was implied that the numerical results were correct. For example, the

*See Appendix B in Hebenstreit and Reid (1978) for complete details. 
simulations produced results which indicate that wave refraction techniques based on linear wave theory (Snell's Law) ". . . do not accurately predict the behavior of nonlinear dispersive waves in conditions of shoaling and refraction" (p. 95). Significant differences between linear theory and model

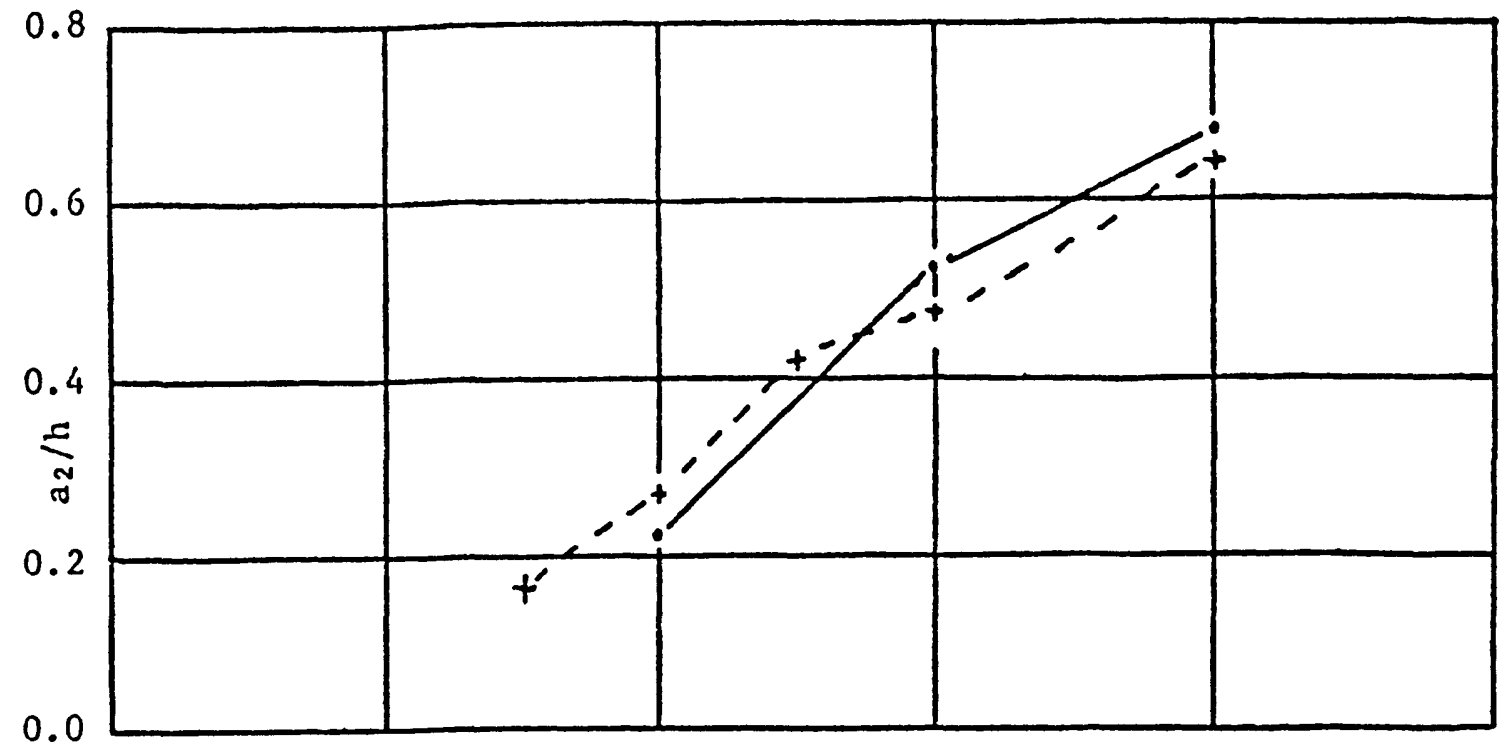

(3)

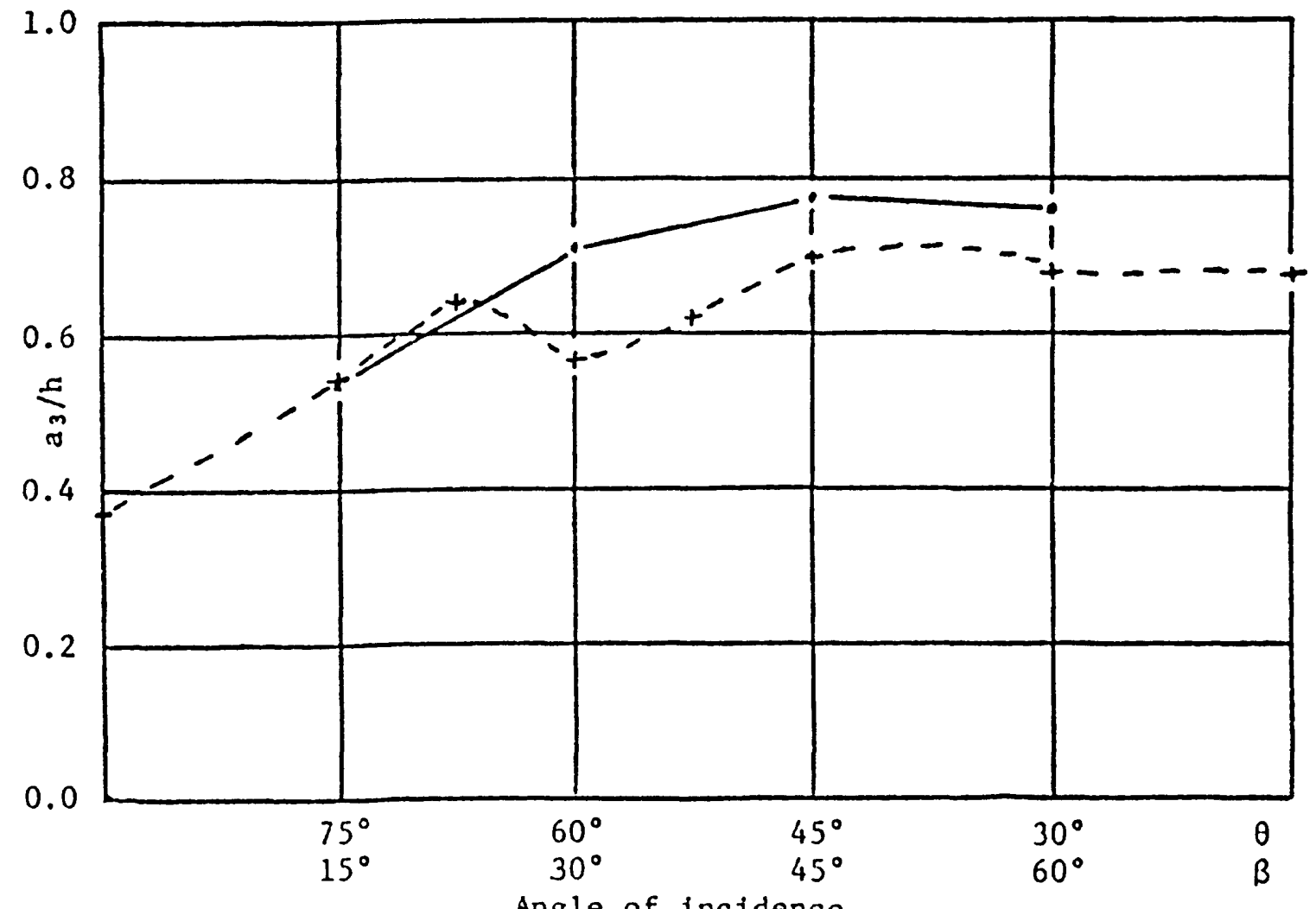

Angle of incidence

(b)

Fig. 6--Values of (a) $a_{2} / h$ and (b) $a_{3} / h$ as a function of incident angle for Perroud's study (dotted lines) and numerical study (solid lines) of Hebenstreit and Reid (1978). 
calculations were evident. The lack of appropriate laboratory and field data for refraction prohibited the authors from directly concluding that their numerical procedures were more correct.

Some quantitative agreement between the numerical study (solid lines) and the experimental data (dotted lines) from Perroud (1957) for wave reflection is evident in Figure 6 . Here, $a_{2}$ is the reflected wave amplitude and $a_{3}$ is the stem amplitude for solitary wave reflections at angles less that $45^{6}$. In this case the numerical model proved to be an excellent tool to study the physical and qualitative behavior of the mach-stem reflection and energy transfer process.

A major breakthrough occurred when Abbott, Petersen and Skovgaard (1978 $a, b)$ subtracted out the truncation errors to make their scheme truly higher-order accurate. Consequently, for the first time, the true dispersive (and dissipative) effect of the mixed derivative term (Boussinesq term) was realized in the computation. As pointed out by Abbott (1979), the truncation error can, of course, be reduced by using a very large number of grid points per wave length in both time and space. For example, the stanford group (Street, Chan and Fromm, 1970) for a 12 second wave ( $\approx \approx 100 \mathrm{~m}$ ) used $\Delta \mathrm{x}=4 \mathrm{~m}$ and $\Delta t=0.2 \mathrm{sec}$ for distance and time steps, respectively. But this approach requires unrealistic computation costs since excessively large numbers of grid points and time steps are required for even modest prototype time scales. The more practical approach adopted by Abbott, Petersen and Skovgaard $(1978 \mathrm{a}, \mathrm{b})$ was to reduce the truncation error to an acceptable level so that a relatively coarse grid would still resolve the short wave components with acceptable accuracy.

Two factors that greatly faciliated this effort should also be mentioned here. It was assumed following Long (1964), that all derivatives of third and higher order can be rewritten by means of the first order relations and and still maintain third order accuracy. This leads to Equation(s) (126) needed to convert the higher-order time derivatives to space derivatives and mixed derivatives of the Boussinesq type. Secondly, it was found algorithmically efficient to make this conversion and combine the truncation error with the Boussinesg term so that it could all be subtracted out at the same time.

For example, consider a three-level scheme in time, $n$ and weighted over grid point $j$ and $j+1$ so that the scheme is centered at $\left(n, j+\frac{1}{2}\right)$. For the local water velocity, $u$, we have

$$
\left(\frac{\partial u}{\partial t}\right]_{j+\frac{1}{2}}^{n}=1 \text { st order approximation }+ \text { T.E.3 }+O\left(\Delta x^{4}, \Delta t^{4}\right)
$$

where

$$
\begin{gathered}
\text { 1st order approx. }=\psi \frac{u_{j+1}^{n+1}-u_{j+1}^{n-1}}{2 \Delta t}+(1-\psi) \frac{u_{j}^{n+1}-u_{j}^{n-1}}{2 \Delta t} \\
\text { T.E.3 }=-\frac{1}{6}\left[\frac{3}{4}\left[u_{x x t}\right]_{j+\frac{1}{2}}^{n} \Delta x^{2}+\left[u_{t t t}\right]_{j+\frac{1}{2}}^{n} \Delta t^{2}\right]
\end{gathered}
$$


and a weighting coefficient, $\psi=\frac{1}{2}$ is normally employed. Note that a two-dimensional Taylor series expansion is needed to obtain the truncation error, T.E.3. But from the linearized, long wave equations of continuity and motion

$$
u_{x x t}=-h_{x t t}
$$

$$
u_{t t t}=-c^{2} h_{x t t}
$$

and substituting in Equation (127) provides a correction term to subtract out

$$
\begin{aligned}
\text { T.E.3 } & =\left[\frac{\Delta x^{2}}{8}+\frac{g h \Delta t^{2}}{6}\right]\left[h_{x t t}\right]_{j+\frac{1}{2}}^{n} \\
& \approx\left[\frac{\Delta x^{2}}{8}+\frac{g h \Delta t^{2}}{6}\right]\left[\left(\frac{h_{j+1}^{n+1}-2 h_{j+1}^{n}+h_{j+1}^{n-1}}{2 \Delta t^{2} \Delta x}\right]-\left[\frac{h_{j}^{n+1}-2 h_{j}^{n}+h_{j}^{n-1}}{2 \Delta t^{2} \Delta x}\right]\right]
\end{aligned}
$$

This form for the truncation error means that the Boussinesq term must also be put into an $h_{\text {xot }}$ form as shown by Equation (17), or (35).

All the efforts to date of the Danish Hydraulic Institute (DHI), Computational Hydraulics Centre, Horsholm Denmark (Michael B. Abbott and Associates) have been with three-time-level schemes for the Boussinesq equations. An example of the results for wave shoaling experiments on a gently sloping beach are shown in Figure 7. They are seen to compare quite favorably with experimental values measured in a large wave basin by the U.S. Army Coastal Engineering Research Center as previously shown in Figure 5. This work used the Eulerian form of the equations and a staggered grid similar to that used by all long wave models in two dimensions, (e.g., Leendertse, 1967; Abbott and Ionescu, 1967, etc.).

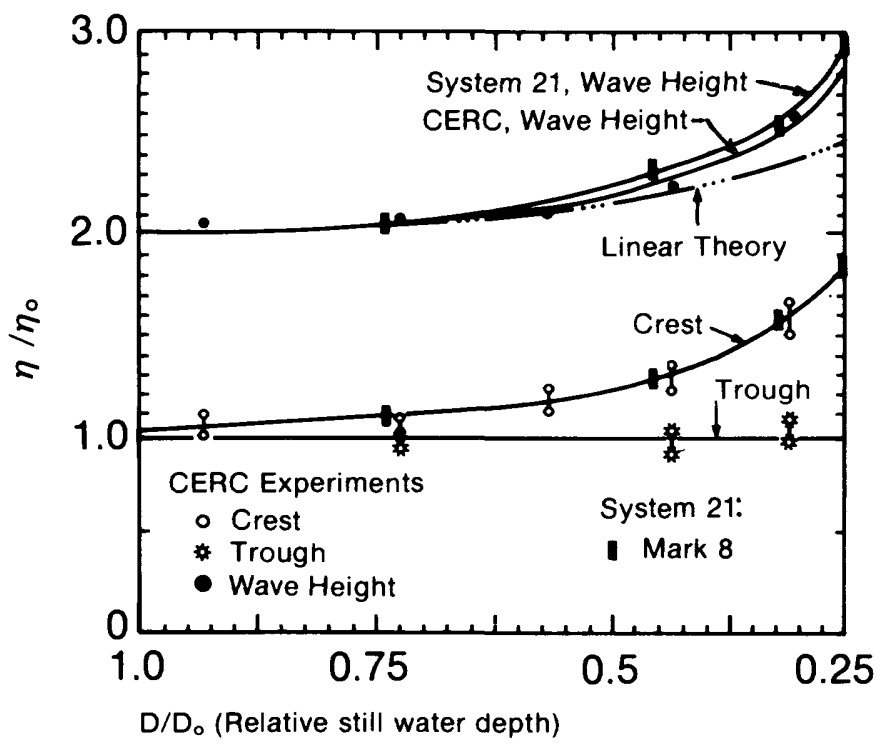

Fig. 7--Comparison of numerical computations of shoaling waves obtained by Abbott et al. $(1978 \mathrm{a}, \mathrm{b})$ in one dimensional as compared with experimental results of Madsen and Mei (1969). 
McCowan (1978) extended this work using the conservation form equations modelled after those of Peregrine $(1966,1967)$ and a compact, fully dense scheme based on the Preissmann (1961) "box" operator but with three time levels as illustrated in Figure $8(\mathrm{~b})$. Similar numerical results were obtained for wave shoaling as those shown in Figure 7. McCowan (1978) commented that the expected development of two divergent solutions common to all three level schemes due to error accumulation at alternate time levels (Abbott, 1979, p. 203) appeared as only ". . . minor oscillations" in early tests. This was felt due to the third derivative Boussinesq term operating at all three time levels and "binding" the alternate levels together.

Further study of the three-level Preissmann scheme is presently in encountered in using the three-level box scheme for the Boussinesq equations. Further discussion by Jensen (1981) revealed that additional "averaging" procedures were needed by McCowan to suppress the oscillations. The reasons for this are presently under investigation. Abbott (1979) states that

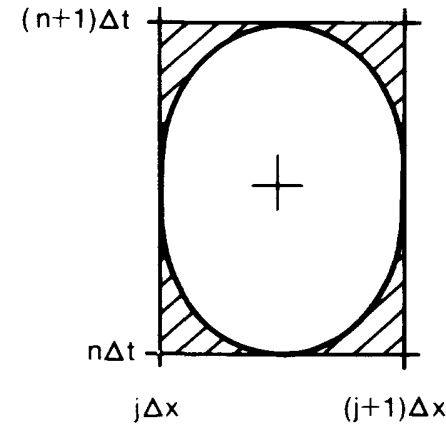

(a) Two-Levels

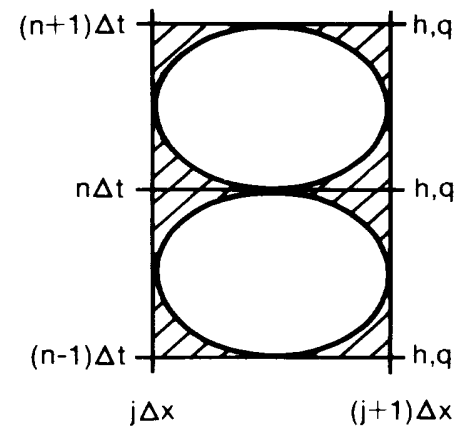

(b) Three-Levels

Fig. 8--The implicit Preissman or "Box" scheme for (a) two-time levels or (b) three-time levels as needed for the Boussinesq equations.

". . smooth and slowly varying (oscillations). . occur, in fact, in any such (three level) scheme and necessitate(s) a periodic realignment of the two schemes through the use of dissipative interface."* (p. 203).

Such practices for the Boussinesq-type equations could again negate the influence of the third derivative term.

The most recent effort was briefly reported by Haugel (1980) in which higher-order-difference methods were applied to his equations $(80,81)$. Details were unavailable since this reference was only the abstract-summary of the full article, yet to be published.

*A "dissipative interface" is equivalent to locally averaging adjacent values which in turn means introducing numerical damping to smooth or filter out the unwanted oscillations. 
other possibilities exist to devise higher-order accurate, finitedifference approximations of the Boussinesq equations.

For example, because of the inherent oscillation problems associated with three-level schemes, and the inaccuracies resulting from specifying the initial conditions at two time levels, it would appear prudent to use a two-level scheme for time marching. Consequently, the Boussinesq term must be in the form $u_{x x d}$ so that three space grid points are fundamentally required to form the second derivative. A Crank-Nicholson implicit-type operator is indicated which must also be compatible with a tri-diagonal algorithmic structure for economy in numerical solution. Some type of predictor-corrector procedure is indicated and consistent with the need to iterate between time steps for the nonlinear convection term.

The convective term, uu would normally be central-differenced for increased accuracy. Such procedures can creat "wiggle" instabilities in propagation-type problems as analyzed and discussed in great detail by Leonard (1979) for the advection-diffusion equation. Such "zig-zagging" instabilities have also been reported for the 2-D long wave equations by Abbott (1979), pp. 259-260) using a staggered operator and by Hill (1981) using the Preissmann scheme. Roache (1977) gives a complete review. Wiggles are produced by central differencing the convection term so that the value of the difference is independent of variations at its midpoint. This leads to what Leonard (1979) labeled "convective insensitivity." For the advection-diffusion equation and Peclet number,$P_{\Delta}$ is defined as

where

$$
\mathrm{P}_{\Delta}=\frac{\gamma}{\Gamma}
$$

$$
\begin{aligned}
\gamma & =\text { the courant number, and } \\
r & =\text { the dimensionless dispersion coefficient } \\
& =k \Delta t / \Delta x^{2} . \\
K & =\text { the dimensional diffusion (dispersion) coefficient }
\end{aligned}
$$

when

$$
P_{\Delta} \leqslant 2
$$

wiggle instabilities are suppressed. This is not always practical and the most popular alternative is to use first-order upstream (upwind) differencing for the convection term. However, the artificial, numerical diffusion of such methods leads again to low accuracy for diffusion. In some well-documented instances (e.g. Roache, 1977) the numerical diffusion from the truncation error terms is significantly larger than the physical diffusion being modeled.

*Roache (1977) used the definition cell Reynolds number instead of the more common Peclet number. 
The similarity between a diffusion term of second order, and the Boussinesq term approximated in Equation (124) should be obvious. Consequently, Leonard (1979) developed a higher-order accurate, upstream differencing algorithm called QUICKEST. It avoids the wiggle problem of central differencing, yet remains free of the inaccuracies associated with simple upstream differencing. The method is based upon a quadratic upstream interpolation procedure that has been formally proven to be identical to a forward-time, centered-space (FTCS) scheme with truncation errors subtracted out and all third derivatives in space centered at $\left(j-\frac{1}{2}\right)$ rather than $j$. (Basco, 1982). The explicit algorithm of Leonard (1979) is being extended to an implicit scheme of similar structure and its properties studied for use in transport-dispersion problems (Basco, 1982). The implicit Leonard algorithm could be readily adapted for the Boussinesq-type equations.

\section{Filter Scheme Methods}

Filter-scheme methods are numerical convolution techniques that are fundamentally different from the finite-difference or finite-element methods. If the response function, relating output to input is known for a system, then by convoluting the known variable(s) over the space domain by a suitable weighting function, the unknown variables result as successive numerical convolution processes. The proper weighting function is the Fourier transform of the response function. Details are presented in Basco (1981) and Reid and Basco (1982) for the pure advection case with one dependent variable.

With two unknowns, such as $u, h$ in the long wave or Boussinesq, 1-D system, the weighting function becomes a $2 \times 2$ matrix that requires four independent response functions and corresponding Fourier transform equations for solution. The four response functions can be readily determined from the complex wave propagation factor, $\theta$ and Equation (121). Further datails are presented in Basco (1981). A sample numerical calculation of an example bivariate problem has yet to be conducted. The computational effort will be considerably greater than for the single variable case. In theory, nonlinear equations require recalculation of response functions, and their Fourier Transforms before each new convolution (time step). Irregular bathymetry and cross-sections further increase the complexity of the method. However, numerical accuracy can be made extremely high for very short, steep waves such as a step function. The filter-scheme method could then become a very accurate tool (numerical river flume or wave basin) for use in testing and calibrating other, more economical, finite-difference methods. 
The purpose of this study was to review and summarize the state-ofknowledge concerning rapidly-varied, unsteady free surface flows and numerical computation techniques for problem solution. Based upon the information included in this report, the following conclusions are drawn and recommendations offered.

1. No single derivational method of the major types reviewed can be considered as the "correct" procedure.

2. For small amplitude waves $\left(\epsilon \cong \sigma^{2} \ll 1\right)$ on a horizontal bottom, all derivations reduce to the same Boussinesq equations given by Equations (1) and (2).

3. For finite-amplitude waves (using Equation (36) for the vertical velocity distribution) major differences result in the kinds and numbers of additional terms that appear in the motion equation. Through continuity, they may be equivalent but this was not confirmed by this study.

4. Further differences appear among the equations for variable water depths in which many additional terms result.

5. The scaling procedure employed to discern the relative magnitude of all the terms proved inconclusive. While confirming that the advection term and primary Boussinesq term $\left(b_{1}\right)$ were relatively equivalent $\left(\epsilon \cong \sigma^{2}\right)$, it also showed how other terms $\left(b_{2}\right)$ including derivative product terms were of this same scale. This contradicts earlier scaling results of Peregrine (1967) and Whitham (1974). The scaling procedure must be reevaluated.

6. The perturbation analysis confirmed the significant reduction in celerity of shorter wave components caused by the primary Boussinesq term (small amplitude theory).

7. The full (small amplitude theory) Boussinesq equations are required for Froude numbers greater than 0.05 to compute celerities of engineering significance (5\% accuracy).

8. The secondary Boussinesq term ( $b_{2}$, see Equation 91 ) is an incorrect addition by itself to the small amplitude, Boussinesq equations. It is tentatively concluded that all other derivative product and third space derivatives must be combined with this term to produce the desired finite-amplitude effects.

9. The small perturbation analysis method utilized herein breaks down when used to study the higher-order terms since they also are neglected in this analysis procedure.

10. The Chezy or Manning resistance formulas must be replaced by more general expressions to include frictional effects for short-wave components. 
11. Small variations in the momentum flux incident upon a short wave system can have a very great effect upon the celerities, wave lengths and wave amplitudes.

12. Great care must be exercised to eliminate truncation errors from finite-differnce schemes of the Boussinesq equations. Truncation errors can cause numerical amplitude and frequency dispersion to swamp the significance of the Boussinesq term(s).

13. Three-time-level schemes used in many numerical simulations today require some type of periodic averaging process to eliminate diverging solutions and oscillations at alternate time steps. This may also contribute to inaccuracies in solutions at Boussinesq-type equations.

14. Filter-scheme methods offer an alternative way to solve the smallamplitude Boussinesq equations with great accuracy.

Although significant clarification remains to determine the best equation for finite-amplitude flows over variable topography, it is strongly suggested that initial numerical work be undertaken to demonstrate the usefulness of solving the small-amplitude Boussinesq equations for selected, practical hydraulic engineering problems. For this purpose, the finite-difference method is preferred due to its general usage by the hydraulics community. An explicit, two-level higher-order accurate, upstream difference (convection terms) scheme is recommended. This would be for the small-amplitude equation on a constant slope with generalized bed shear term to account for short wave energy losses. The solution of canal surge problems due to sudden, rapid flow releases is one example problem for study. The differences in wave celerities and attenuation of the surge as computed by the long wave versus the Boussinesq-type equations would be the very interesting and practical result of such an investigation. Another example would be the propagation of spiked hydrographs for flashy streams on very steep slopes.

It is also recommended that other scaling methods be studied to further aid in learning the relative importance of all the added terms in the finite-amplitude versions of the Boussinesq equations. Related to this is a careful comparison of the added terms for the variational and conservation methods to determine if in fact they are identical.

Finally, at some early future stage, a very accurate solution of the small-amplitude equations by the Filter-Scheme Method should be attempted. It will definitely serve as check on the accuracy of the finite-difference method. This is especially important because solutions to the Boussinesq equation are extremely sensitive to small variations in the local momentum flux as originally demonstrated analytically by Lighthill and Whitham (1955). 


\section{REFERENCES}

Abbott, M.B. (1966) An Introduction to the Method of Characteristics, American Elsevier, N.Y.

Abbott, M.B. (1979) Computational Hydraulics, Pitman, London.

Abbott, M.B. and F. Ionescu (1967) "Aspects of a Computational Model for Long-Period Water-Wave Propagation, RAND Corp. RM 5294, May.

Abbott, M.B. and G. Rodenhuis (1972) "A Numerical simulation of the Undular Hydraulic Jump," Journ. Hyd. Res. Vol. 10, pp. 239-257.

Abbott, M.B., H.M. Petersen, and D. Skovgaard (1978a "On the Formation and Stability of the Undular Hydraulic Jump," Intl. Hyd. and Sanit. Engng., Rept. Series No. 10 .

Abbott, M.B., H.M. Petersen, D. Skovgaard (1978b) "Numerical Modelling of Short Wave in Shallow Water," Journ. Hyd. Res., Vol. 3.

Barnes, H.H.J. (1967) "Roughness Characteristics of Natural Channels," U.S. Geological Survey. Water-Supply Paper No. 1849.

Basco, D.R. (1981) "Filter-Scheme Numerical Methods for Solution of Wave Propagation Problems," U.S. Geological Survey Open-File Report 81-910.

Basco, D.R. (1982) "An Implicit, Wiggle-Free and Accurate Upstream Finite-Difference Algorithm for the One-Dimensional Transport-Diffusion Equation," USAE, Waterways Expt. Station, Environmental Laboratory, Final Report, (DACW39-81-M-4377), in preparation.

Benjamin, T.B. and M.J. Lighthill (1954) "On Cnoidal Waves and Bores," Proc. Royal Sec. (London) A. Vol. 224, p.448.

Berlamont, J. (1976) "Roll-Waves in Inclined Rectangular Open Channels," Inter. Sym. on Unsteady Flow in Open Channel, BHRA, Newcastle-upontype, England, April 12-15.

Bijker, E.W. (1966) "The Increase of Bed Shear in a Current Due to a Wave Motion," Proc. 10th Coastal Engng. conf., Vol. I, pp. 746-765.

Boussinesg, J.V. (1872) "Theory of Waves and surges which Propagate the Length of a Horizontal Rectangular Canal, Imparting to the Fluid Contained Within the Canal Velocities that are Sensibly the Same from Top to Bottom," Jour. Math. Pures Appliquees, Series 2, Vol. 17, pp. 55-108.

Chow, V.T. (1959) Open-Channel Hydraulics, McGraw-Hill, N.Y.

Churchill, R.V. (1962) Fourier Series and Boundary Value Problems, McGrawHill, N.Y. 
Cosserat, E. and F. Cosserat (1909) Theorie des corps deformables,

A. Hermann \& Fils, Paris.

Cunge, J.A. (1969) "On the Subject of Flood Propagation Computation Method (Muskingum Method)," Journ. Hyd. Res., Vol. 7, pp. 205-230.

Cunge, J.A., F.M. Holly Jr., and A. Verwey (1980) Practical Aspects of Computational River Hydraulics, Pitman, Boston.

Dressler, R.F. (1978). "New Nonlinear Shallow-Flow Equations with Curvature," Journ. Hyd. Res., Vol. 16, no. 3, pp. 205-222.

Green, A.E., N. Laws, and D.M. Naghdi (1974) "On the Theory of Water Waves" Proc. Royal Soc. (London), vol. 338, pp. 43-55.

Green, A.E. and P.M. Naghdi (1976a) "A Derivation of Equations for Wave Propagation in water of Variable Depth," Journ. Fluid Mech., Vol. 78, part 2, pp. 237-246.

Green, A.E. and P.M. Naghdi (1976b) "Directed Fluid Sheets," Proc. Royal Soc. (London), vol. 347, pp. 447-473.

Hammack, J.L. (1973) "A Note on Tsunamis: Their Generation and Propagation in an Ocean of Uniform Depth," Journ. Fluid Mech., Vol. 60, part 4, pp. 769-799.

Haugel, A. (1980) "Adaptation of Tidal Numerical Models to Shallow Water Wave Problems," 17th CEC, Sydney, March, pp. 277-278, (Abstract only).

Hebenstreit, G.T. and R.O. Reid (1978) "Reflection and Refraction of Solitary Waves--A Numerical Investigation," Dept. of Oceanography, Ref. 78-7-T, Texas A\&M University, College Station, July.

Henderson, F.M. (1966) Open Channel Flow, MacMillan, N.Y.

Hill, J. (1981) "Experiments With a Nonstaggered, Implicit, Finite-Difference Operator for the Two-Dimensional Free Surface Flow Equations, unpublished M.S. Thesis, Texas A\&M Univ., December.

Jensen, R.E. (1981) personal communication, November.

Jonsson, I.G. (1966) "Wave Boundary Layers and Friction Factors," Proc. 10th Coastal Engng. Conf., Vol. I, pp. 127-148.

Kamphuis, J.W. (1975) "Friction-factor Under Oscillatory Waves," Journ. Waterways, Harbours and Coastal Engr., ASCE, Vol. 101, pp. 135-144.

Keller, J.B. (1948) "The Solitary Wave and Periodic Waves in Shallow Water," Commun. Appl. Maths., Vol. 1, pp. 232-339. 
Keulegan, G.H. and G.W. Patterson (1940) "Mathematical Theory of Irrotational Translation Waves," Jour. Res. Nat. Bur. Stand., vol. 24, pp. 47-101.

Korteweg, D.J. and G. de Vries (1895) "On the Change of Form of Long Waves Advancing in a Rectangular Channel, and on a New Type of Long Stabilizing Wave," Phil. Mag., vol. 5, p. 41.

Leendertse, J.J. (1967) "Aspects of a Computational Model for Long-Period Water-Wave Propagation, RAND Corp. RM 5294, May.

Leonard, B.P. (1979) "A Stable and Accurate Convective Modelling Procedure Based on Quadratic Upstream Interpolation, "Comp. Math. in App. Mech. and Engr., Vol. 19, p. 59-98.

Liggett, J.A. (1975) "Basic Equations of Unsteady Flow," in Unsteady Flow In open Channels, Vol. I (ed. by Mahmood, K. and V. Yevjevich). Chapter 2 .

Lighthill, M.J. and G.B. Whitham (1955) "On Kinematic Waves: I. Flood Movement in Long Rivers; II. Theory of Traffic Flow on Long Crowded Roads," Proc. Royal Soc. (London)A, vol. 229, pp. 281-345.

Long, R.B. (1964) "The Initial-Valve Problem for Long Waves of Finite Amplitude," Journ. Fluid Mech., vol. 20, part 1, pp. 161-170.

Longuet-Higgins, M.S. (1974) "On the Mass, Momentum, Energy and Circulation of a Solitary Wave," Proc. Royal Soc. (London)A, Vol. 337, pp. 1-13.

Luke, J.C. (1967) "A Variational Principle for a Fluid with a Free Surface," Journ. Fluid Mech., vol. 27, pp. 395-397.

Madsen, O.S. and C.C. Mei (1969) "Dispersive Long Waves of Finite Amplitude over and Uneven Bottom," MIT, Hyd. Lab., Rept. No. 117.

Madsen, O.S., C.C. Mei, and R.P. Savage (1970) "The Evolution of Time Periodic Long Waves of Finite-Amplitude," Journ. Fluid. Mech., Vol. 44, pp. 195-208.

Mass, W.J. and A.C. Vastano (1978) "An Investigation of Dispersive and Nondispersive Long Wave Equations Applied to Oceans of Variable Depth," Tech. Rept. 78-8-T, Dept. of Oceanography, Texas A\&M Univ., College station, July.

McCowan, A.D. (1978) "Numerical Simulation of Shallow Water Waves," 4 th Australian Conf. on Coastal \& Ocean Engr., Adelaide, Nov., pp. 132-136.

Mei, C.C. and B. LeMehaute (1966) "Note on the Equations of Long Waves over an Uneven Bottom," Jour. Geophys. Res., vol. 71, p. 2.

Meyer, R.E. (1967) "Note on the Undular Jump," Journ. Fluid Mech., Vol. 28, pp. 209-221. 
Naghdi, P.M. (1978) "Fluid Jets and Fluid Sheets: A Direct Formulation," ONR Research Symposium, Spring.

Peregrine, D.H. (1966) "Calculations of the Development of an Undular Bore," Jour. Fluid Mech., Vol. 25, pp. 321-330.

Peregrine, D.H. (1967) "Long Waves on a Beach," J. Fluid Mech., Vol. 27, Part 4, pp. 815-827.

Peregrine, D.H. (1968) "Long Waves in a Uniform Channel of Arbitrary Cross-Section," Journ. Fluid Mech., Vol. 32, Part 2, pp. 353-365.

Peregrine, D.H. (1972) "Equations for Water Waves and the Approximation Behind Them," in Waves on Beaches, (Ed. by R. Meyer) Academic Press, N.Y., pp. 95-121.

Peregrine, D.H. (1976) "Water Waves in Channels," Inter. Sym. on Unsteady Flow in Open Channels, BHRA, Newcastle-upon-Tyre, England, April, paper Â1.

Peregrine, K.H. (1978) "Equations for Water Waves and the Approximations Behind Them," in Waves on Beaches, ed. by R.E. Meyer, Academic Press, N.Y., pp. 95-121.

Perroud, R.H. (1957) "The Solitary Wave Reflection Along a straight Vertical Wall at Oblique Incidence," $U$. of Calif., Inst. of Engr. Res., Tech. Rept. Series 99, Issue 3, Berkeley, Calif., 93 p.

Ponce, V.M. and D.B. Simons (1977) "Shallow Wave Propagation in Open Channel Flow," ASCE, Journ. Hyd. Div., Vol. 103, No. HY12, Dec., pp. 1461-1476.

Preissmann, A. (1961) "Propagation des Intumescences Dans les Canaux et Rivieres, Congres de I'Assoc. Fracaise de Calcul., Grenoble, pp.433-442.

Reid, R.O. (1980) personal communication, February.

Reid, R.O. and A.C. Vastano (1963) "Variational Formulation for Long Gravity Waves in Variable Depth," Trans. AGV, Vol. 44, (Abstracts).

Reid, R.O. and A.C. Vastano (1975) "Linear Quasi-Long Gravity Wave Systems." (unpublished manuscript).

Reid, R.O. And D.R. Basco (1982) "Numerical Convolution for Solution of Wave Propagation Problems: Filter Scheme Methods," Journ. Hyd. Res., Vol. 20, No. 4, pp. 329-349.

Roache, P.J. (1977) Computational Fluid Dynamics, Hermosa, Albuquerque, N.M.

Roberts, B.R. and R.L. Street (1975) "Two-Dimensional, Hydrostatic Simulation of Thermally-Influenced Hydrodynamic Flows," Dept. of C.E., Tech. Rept. No. 194, Stanford Univ., July. 
Starr, V.P. (1947) " Momentum and Energy Integrals for Gravity Waves of Finite Height," Journ. Marine Res., Vol. 6, pp. 175-193.

Stoker, J.J. (1957) Water Waves, Wiley Interscience, N.Y.

Street, R.L. (1976) Simulation of Thermally-Influenced Hydrodynamic Flows-Final Report," Dept. of C.E., Tech. Rept. No. 203, Stanford Univ., January.

street, R.I., R.K-C Chan, and J.E. Fromm (1970) "Two Methods for the Computation of the Motion of Long Water Waves - A Review And Applications," Stanford Univ., Dept. of Civil Engr., Tech. Rept. No. $136,62 \mathrm{pp}$.

Svendsen, I.A. (1974) "Cnoidal waves Over a Gently sloping Bottom," Tech. Univ. of Denmark, ISVA, Series Paper 6.

Ursell, F. (1953) "The Long Wave Paradox," Proc. Cambridge Phil. Soc., Vol. 49, pp. 685-694.

USGS, Water Supply Paper No. 1849 (1977), U.S. Government Printing Office, Washington, D.C.

Vastano, A.J. and J.C. Mungall, (1976) "Theory of Waves and Surges Which Propagate the Length of a Horizontal Rectangular Canal, Etc.," by J.V. Boussinesq, 1972. Department of Oceanography, Ref. 76-2-T, Texas A\&M University, College Station, March, 78 p.

Venezian, G. (1974) "On a Dispersive Theory for Waves in Shallow Water," Look Laboratory, Vol. 4, U. of Hawaii, pp. 22-30.

Venezian, G. (1975) "Nonlinear Dispersive Waves in Shallow Water," Proc. IAHR Congress, Sao Paulo, Brazil.

Venezian, G. (1976) "Further Notes on a Dispersive Theory for Waves in Shallow Water," Dept. of Ocean Engr., Vol. 6, No. 2, U. of Hawaii, July, pp. 35-37.

Whalin, R.W. (1976) "A Variational Approach to Nonlinear Wave Theory," USAE, WES, MP-H-76-16, Vicksburg, Aug. $41 \mathrm{p}$.

Whitham, G.B. (1965) "A General Approach to Linear and Nonlinear Dispersive Waves Using a Lagrangian," Journ. Fluid Mech., Vol. 22, pp. 273-283.

Whitham, G.B. (1967) "Variational Methods and Applications to water Waves," Proc. Roy. Soc. (London), Vol. A299, pp.6-25.

Whitham, G.B. (1974) Linear and Non-Linear Waves, John Wiley, N.Y.

Wiegel, R.I. (1964) Oceanographical Engineering, Prentice-Hall, Englewood Cliffs, N.J. 
APPENDIX

Numerical Test Results for $\gamma_{+}$Celerity Over Range of Cases Given in Table 3 


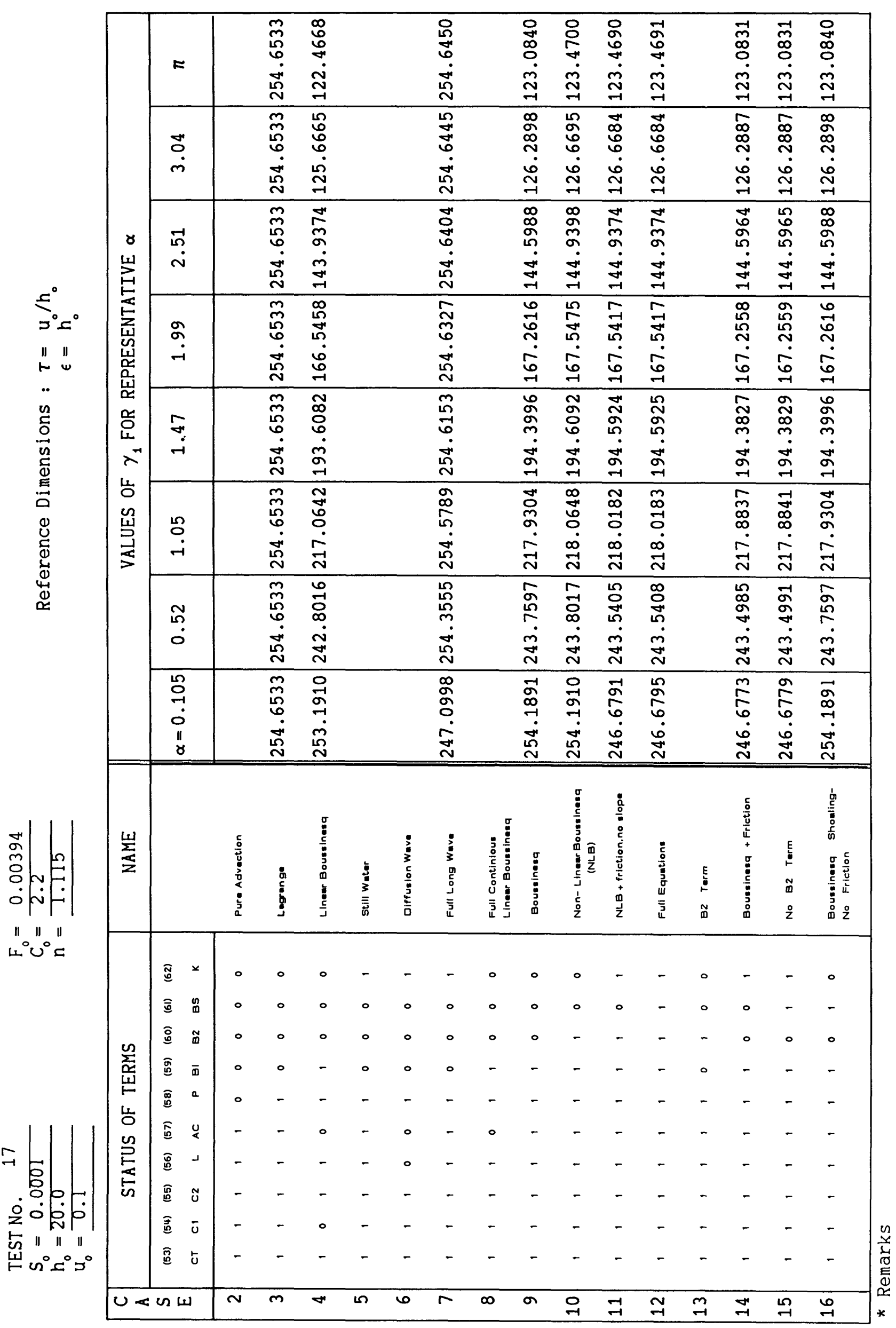




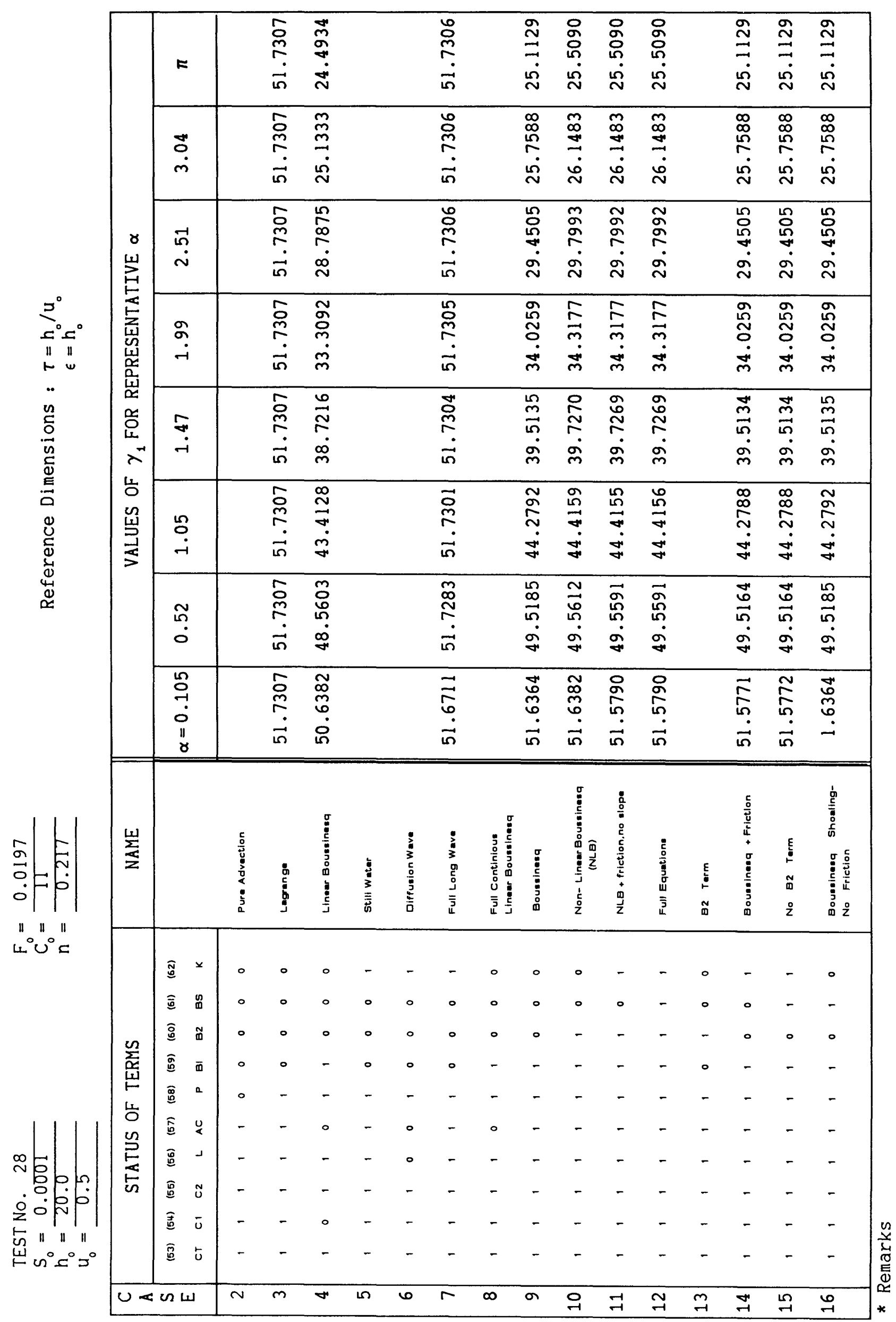




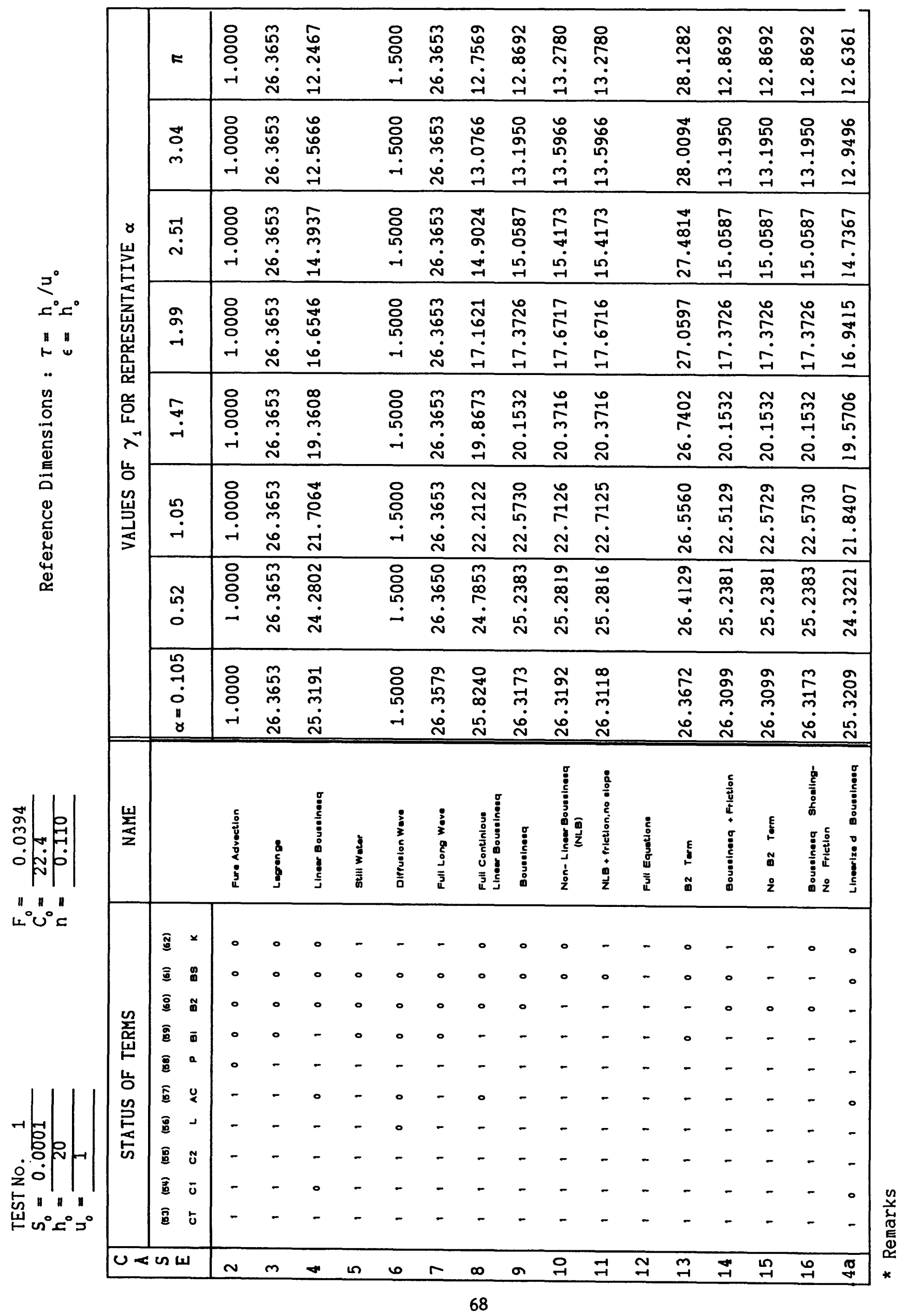




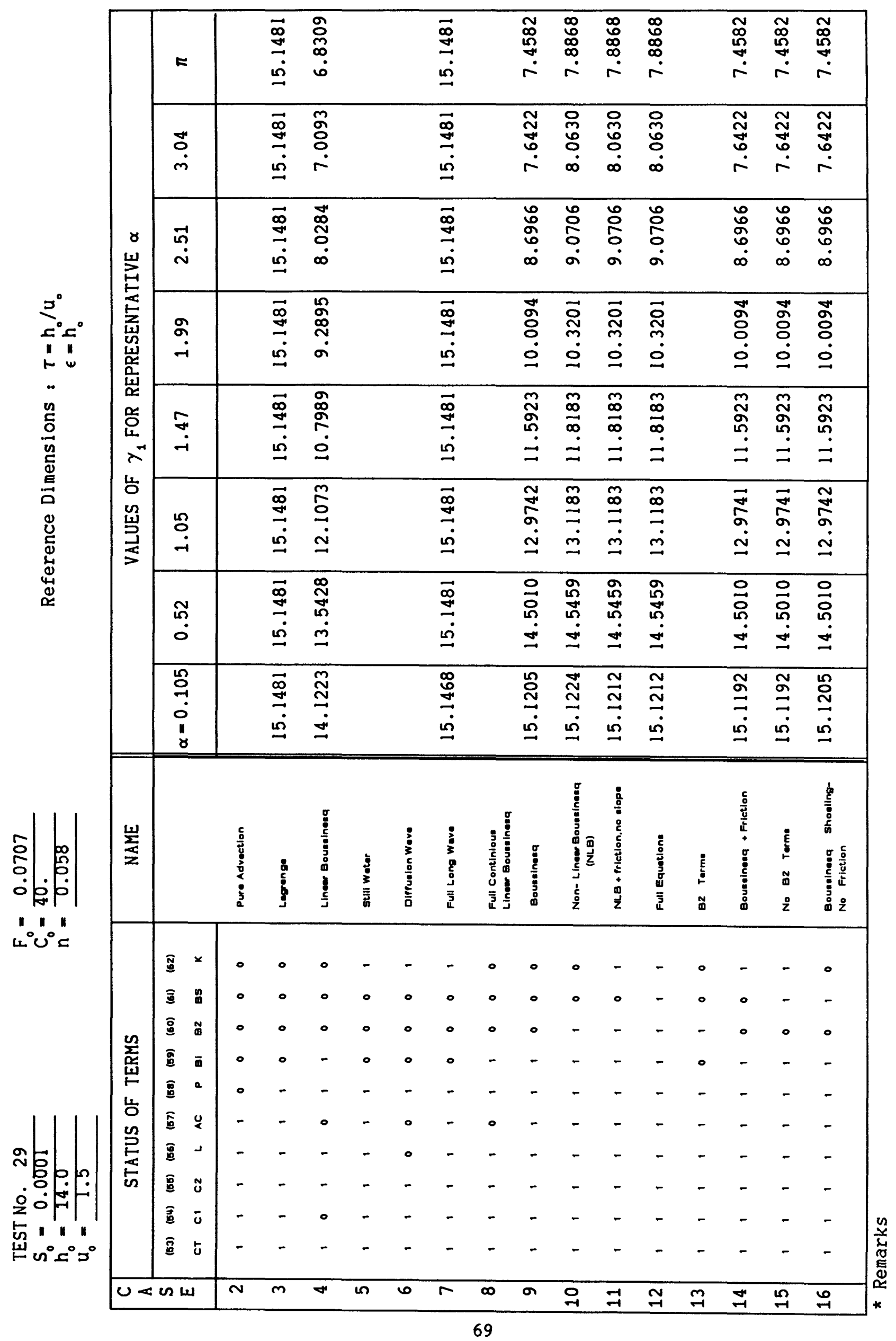




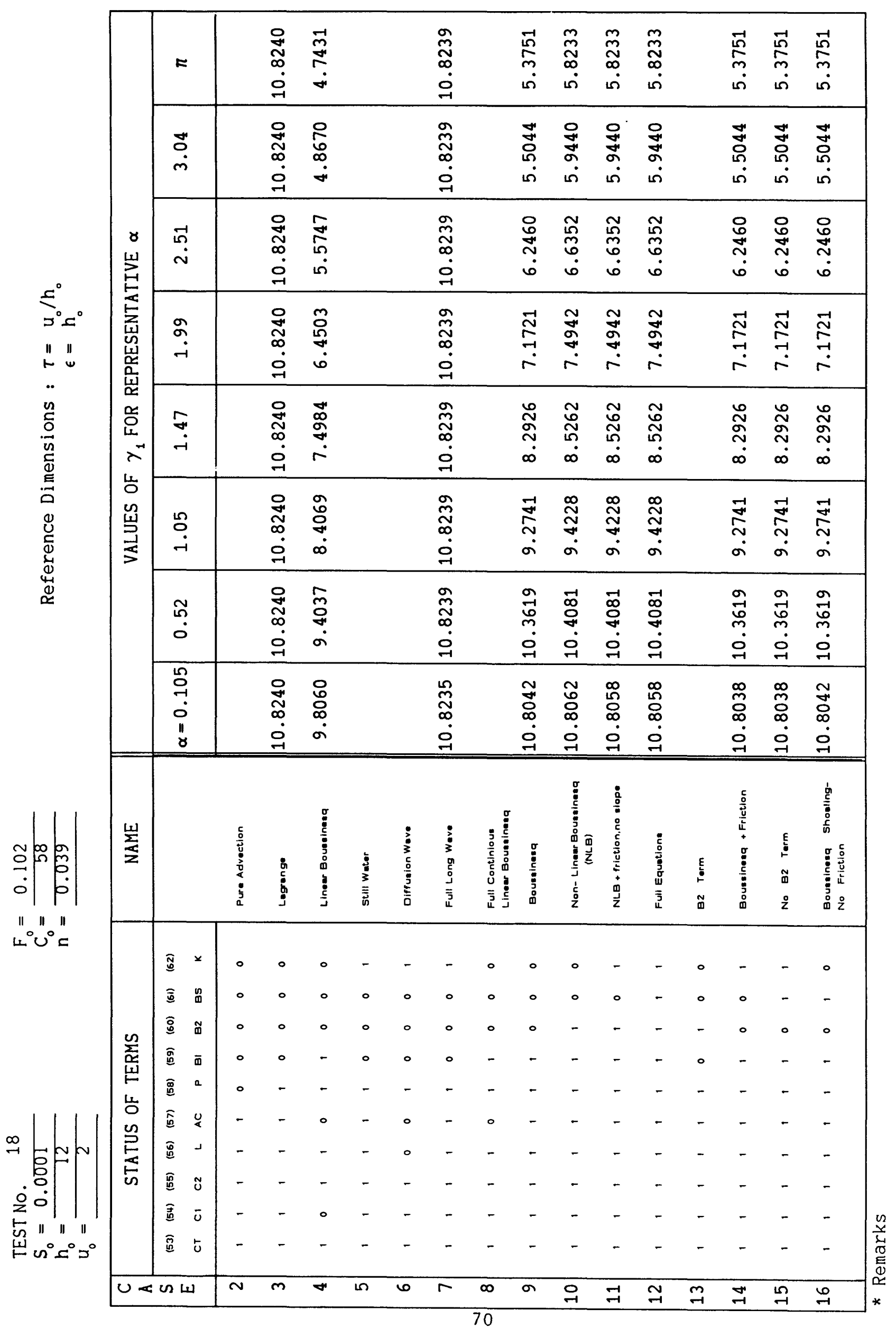




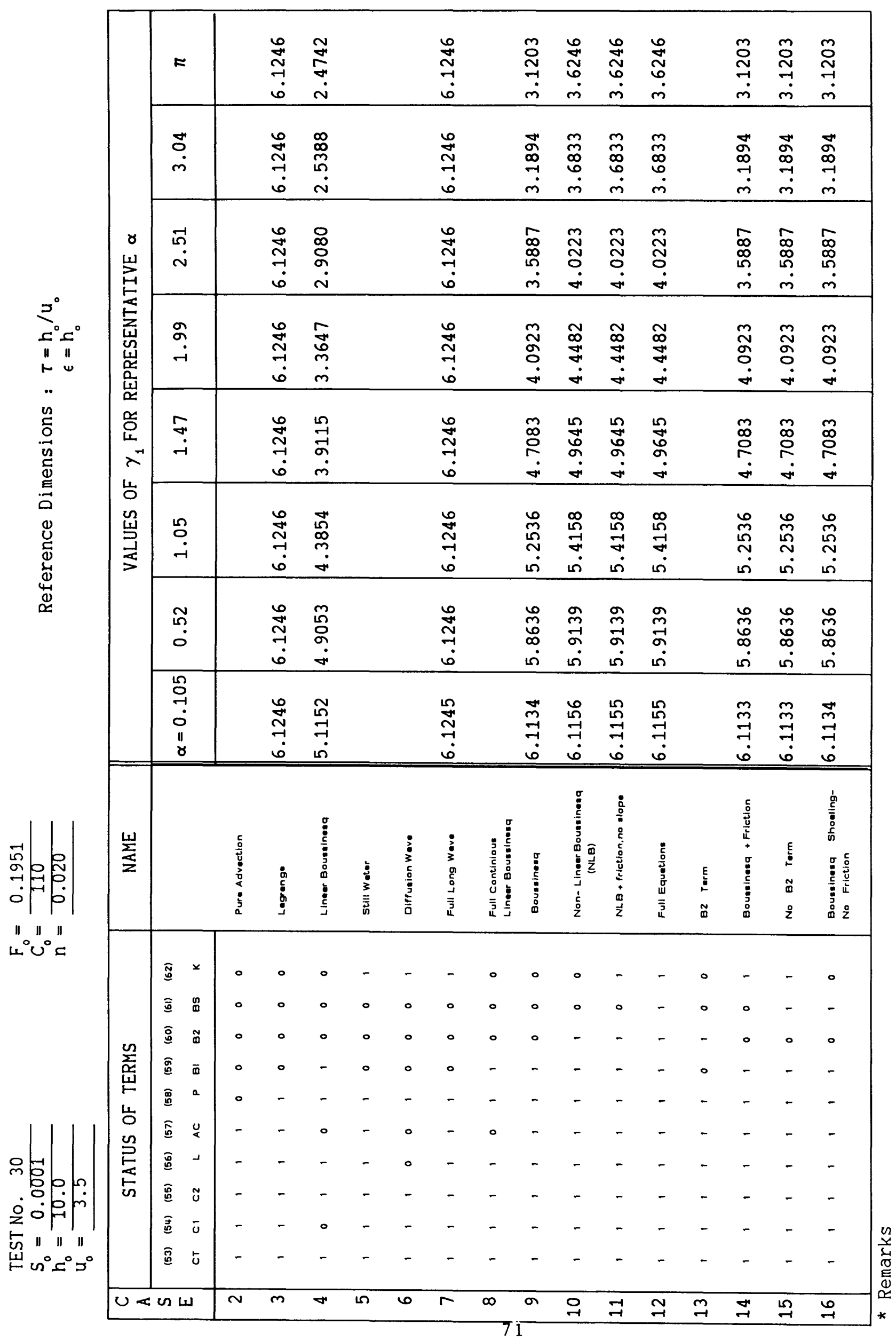




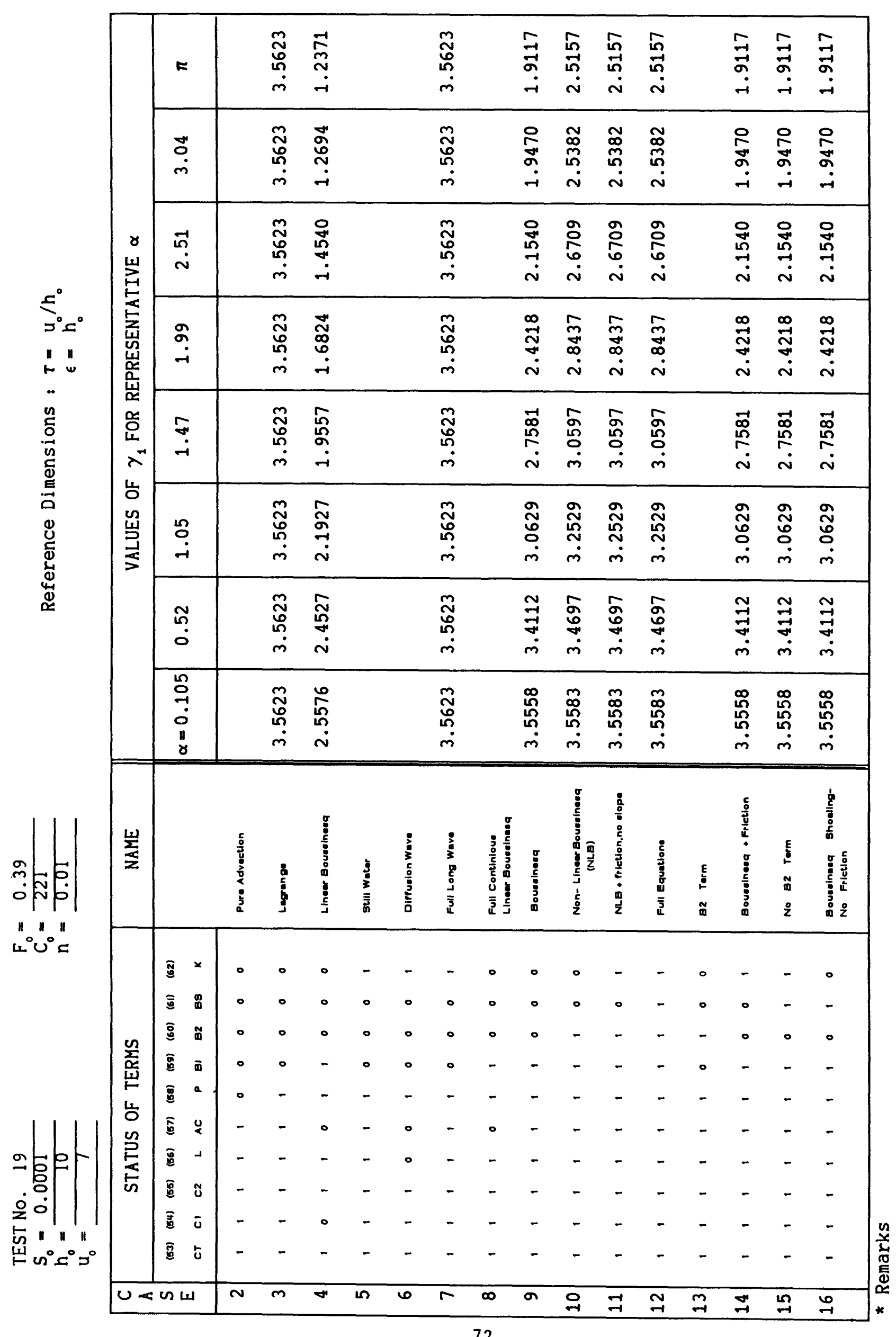




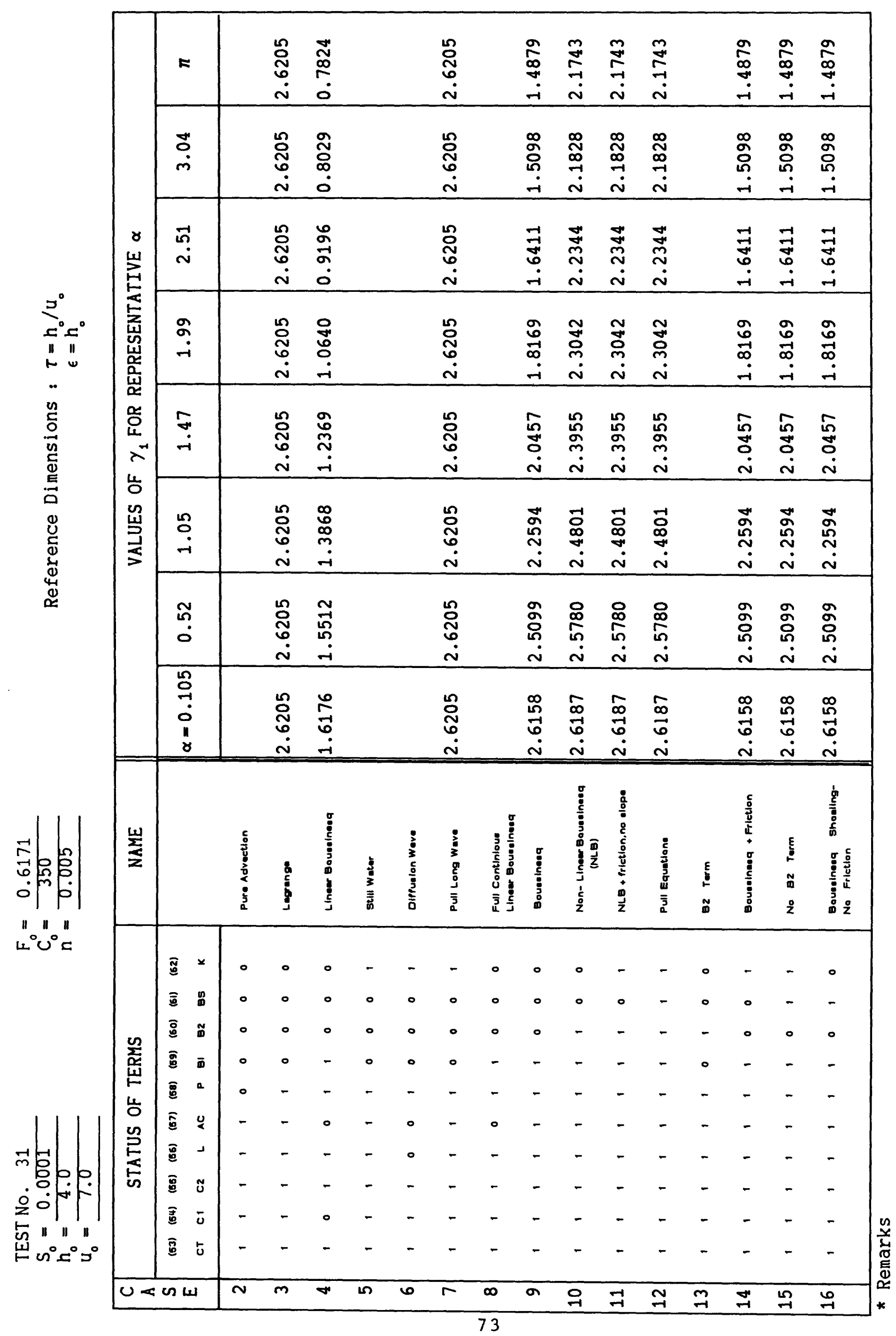




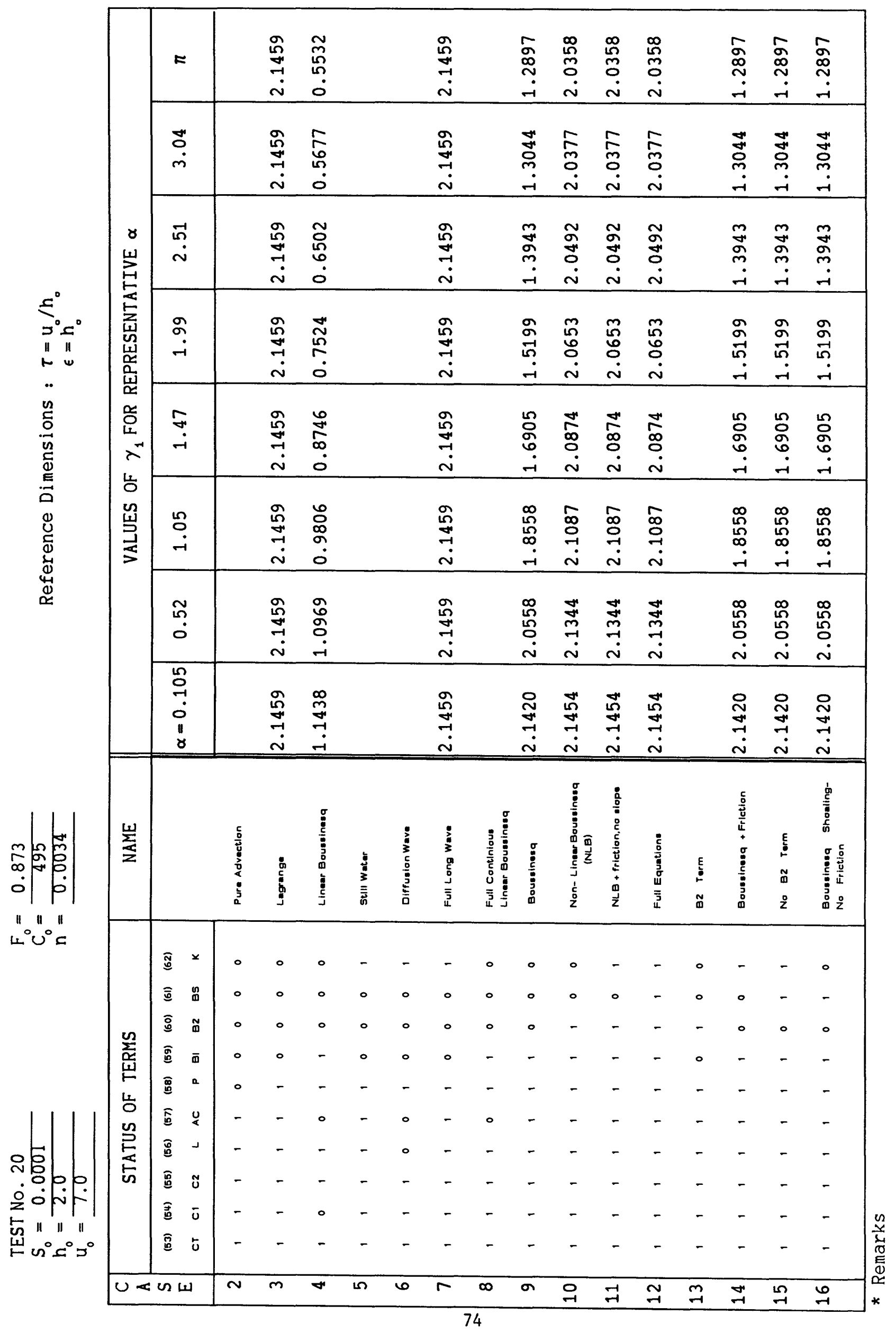




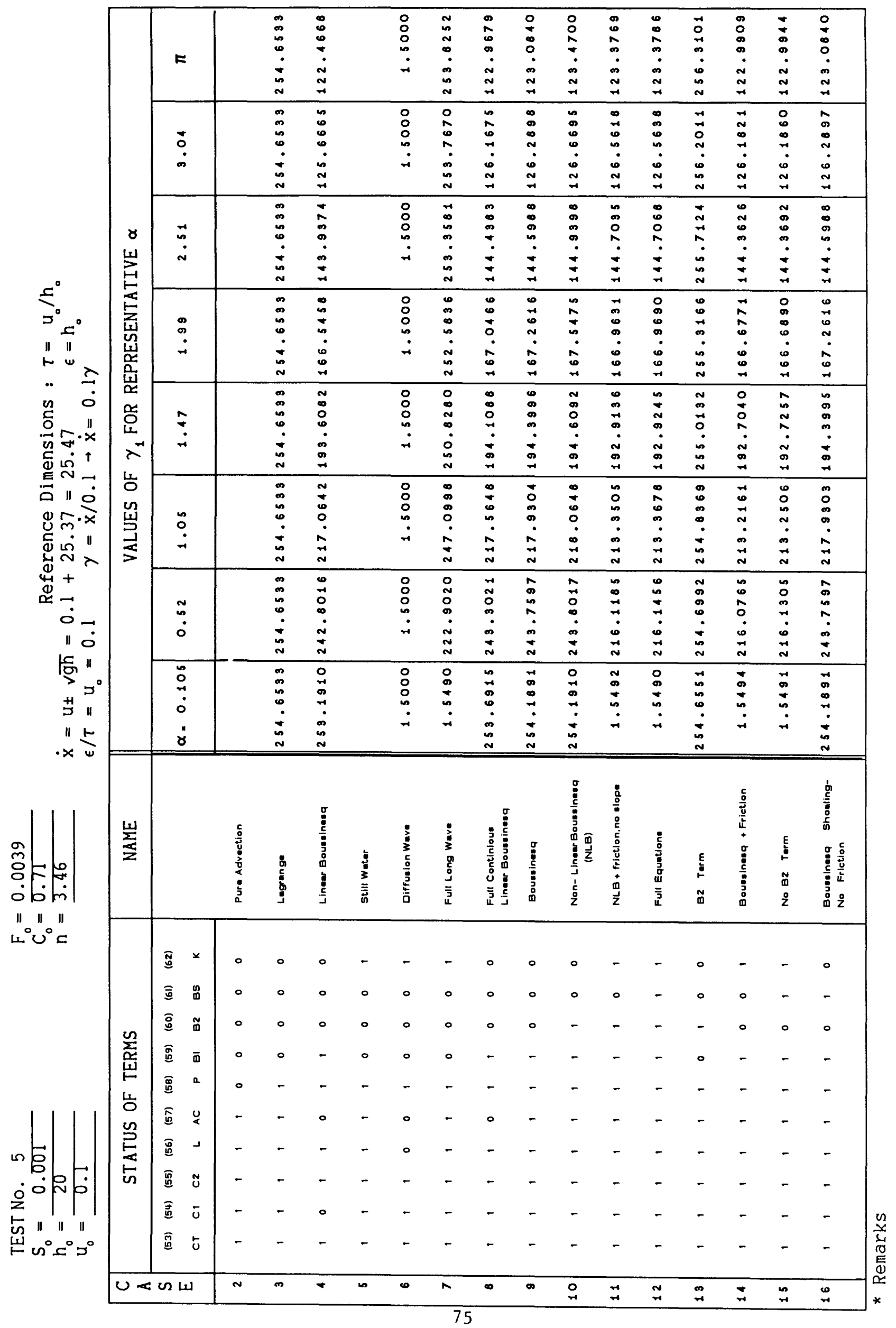




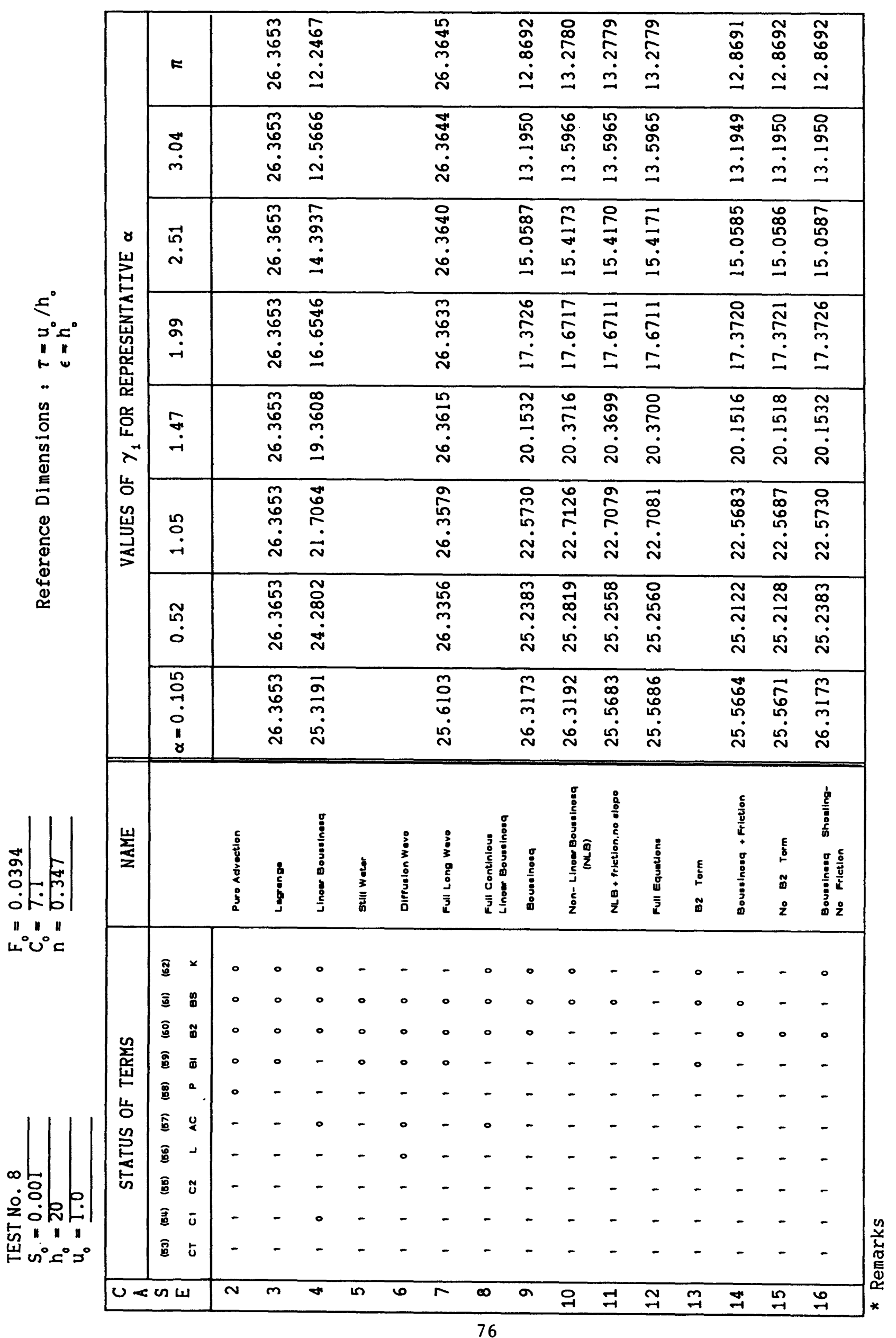




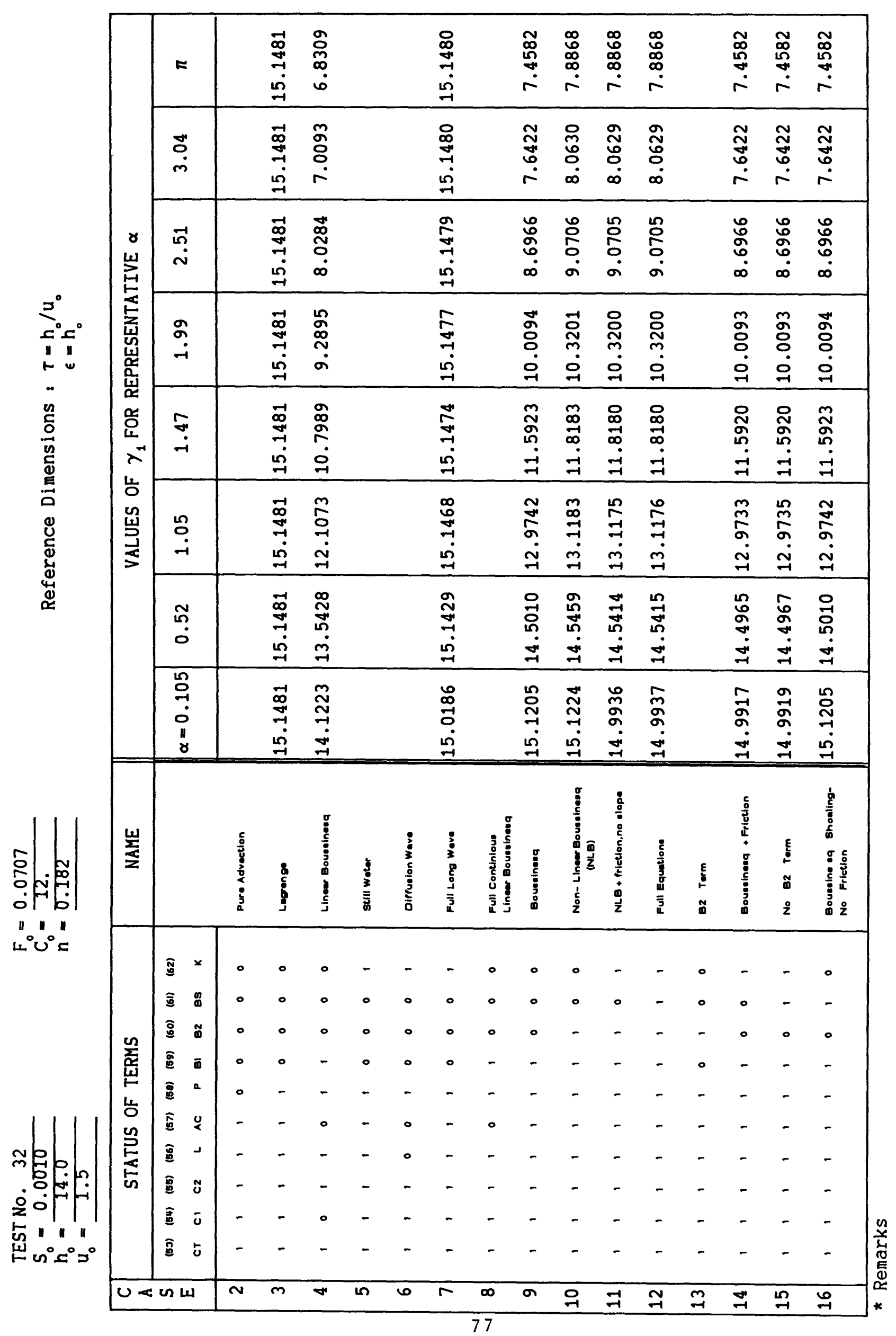




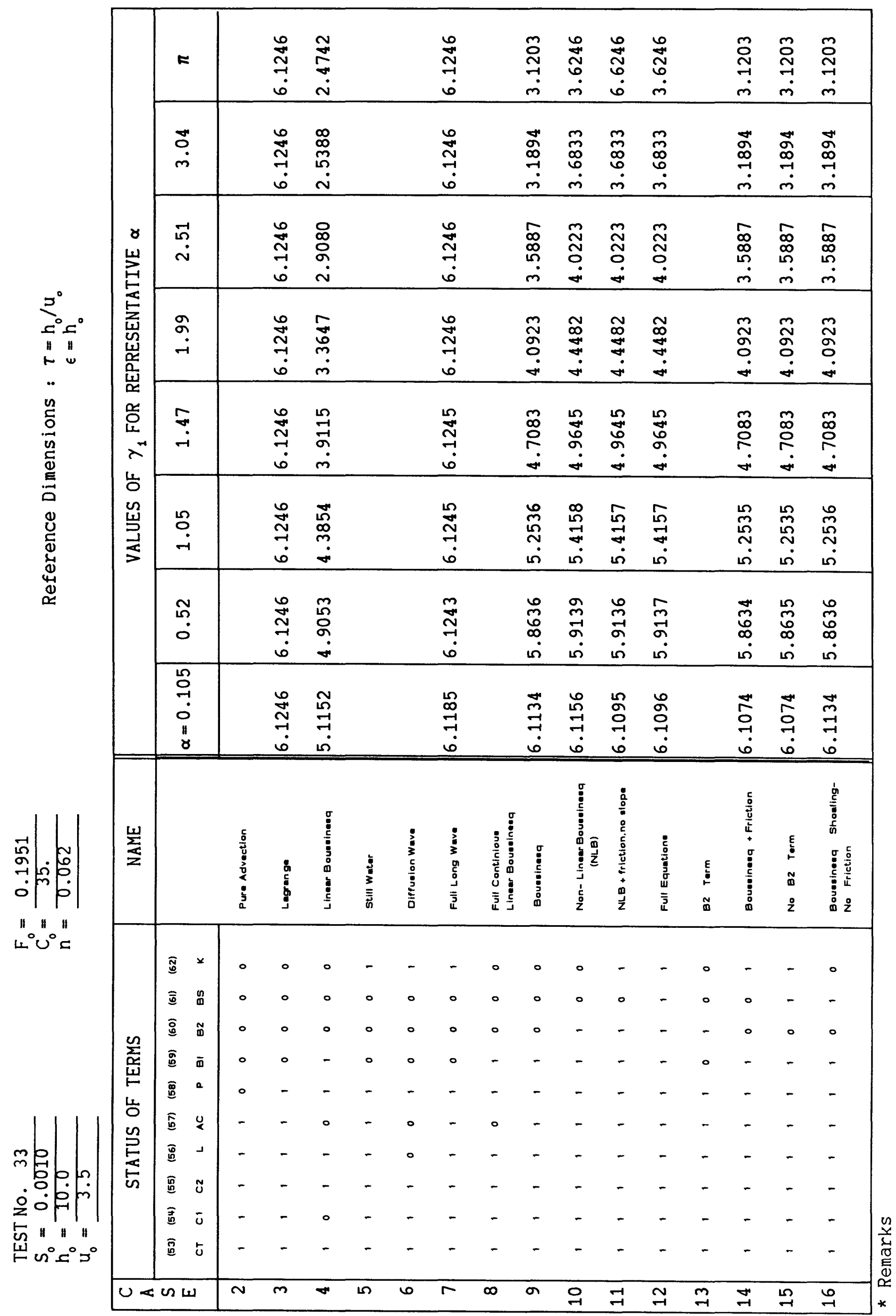




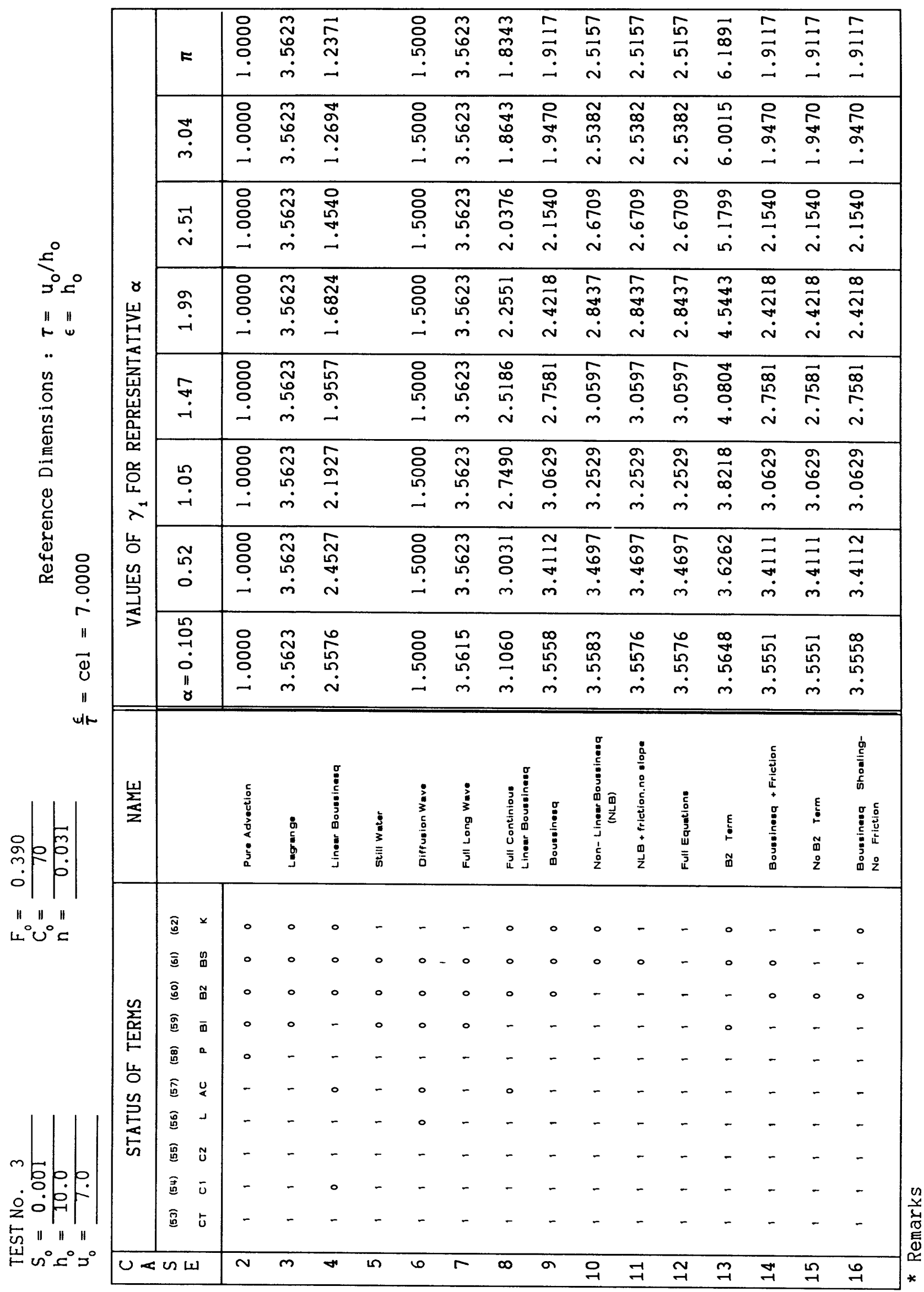




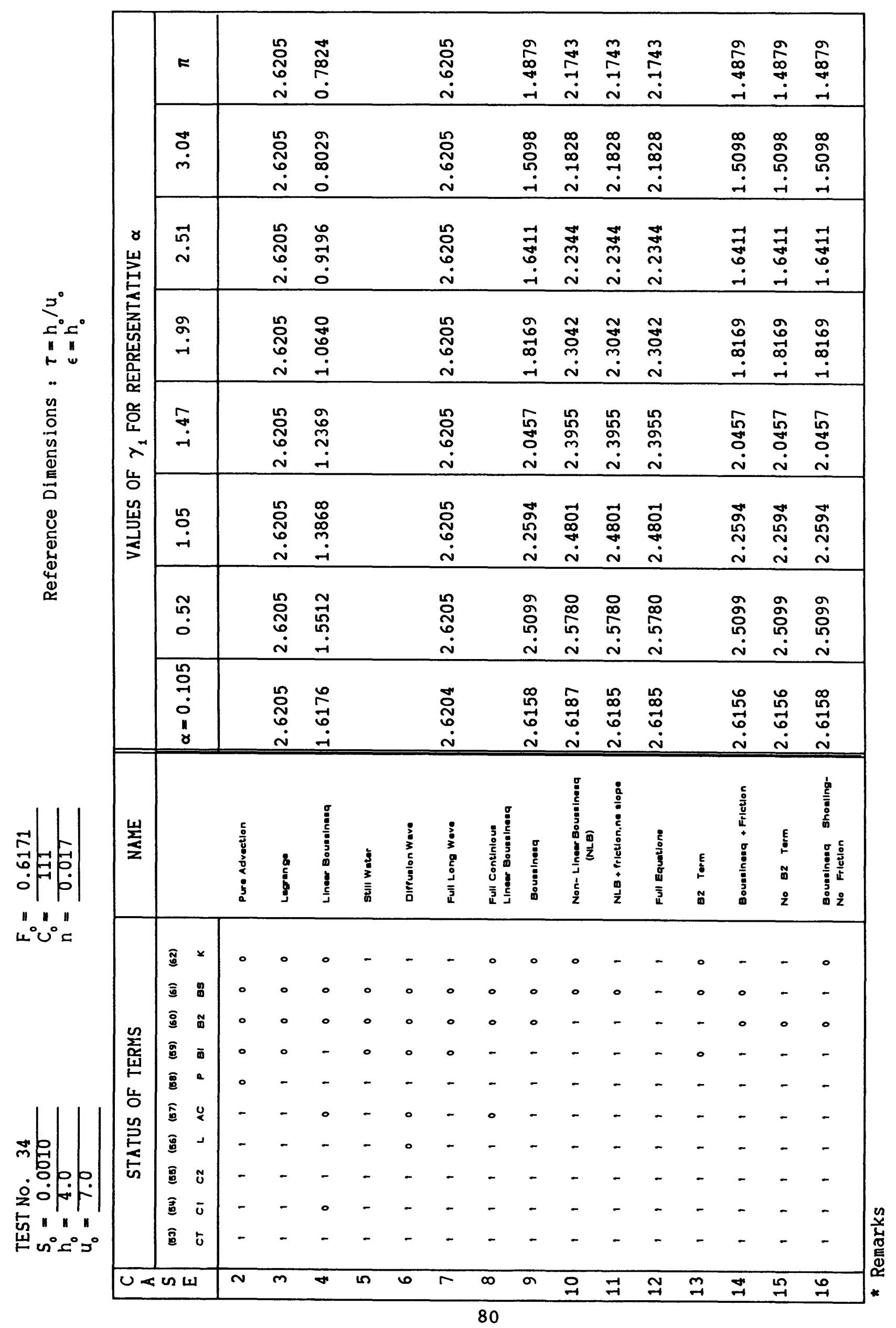




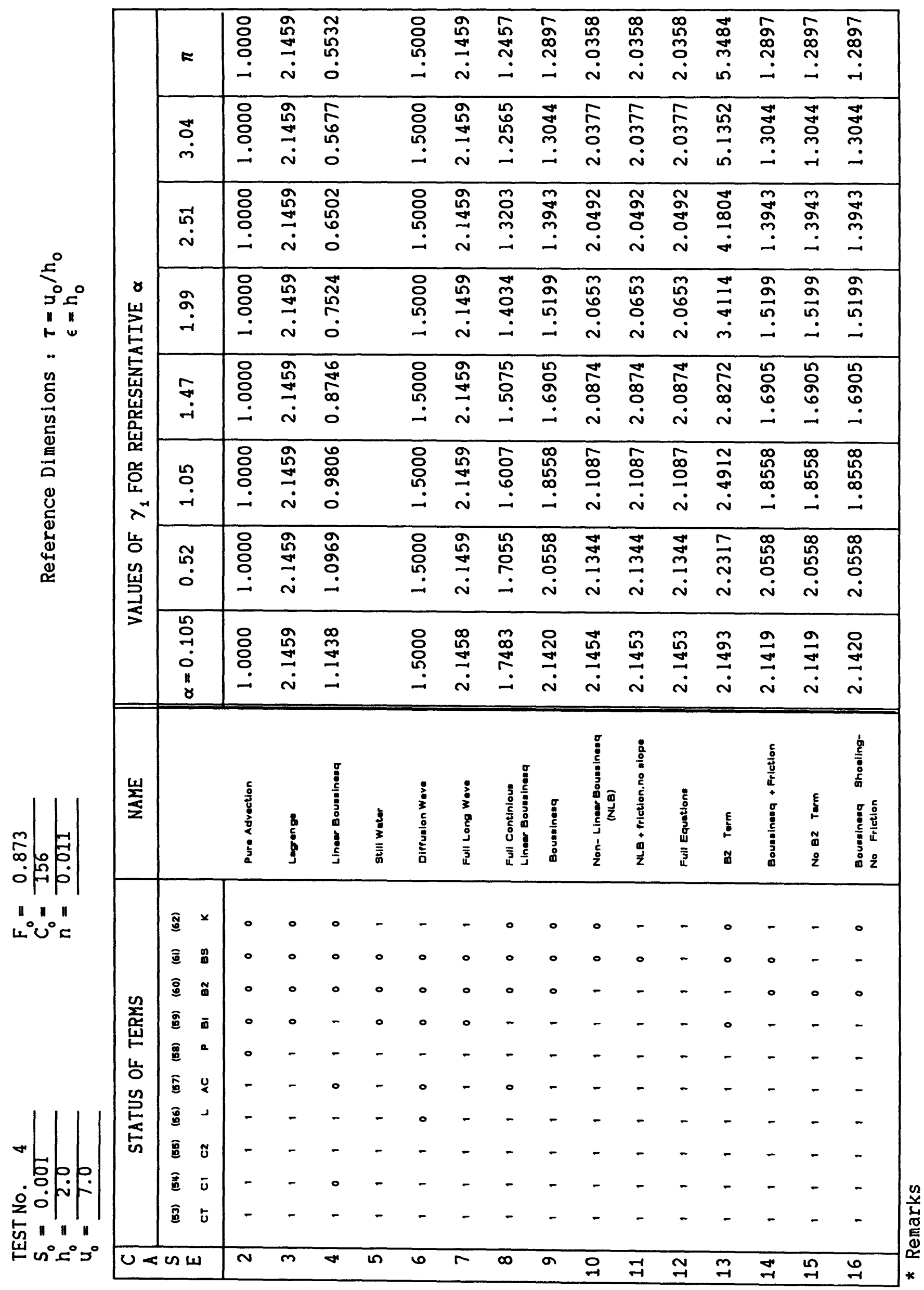




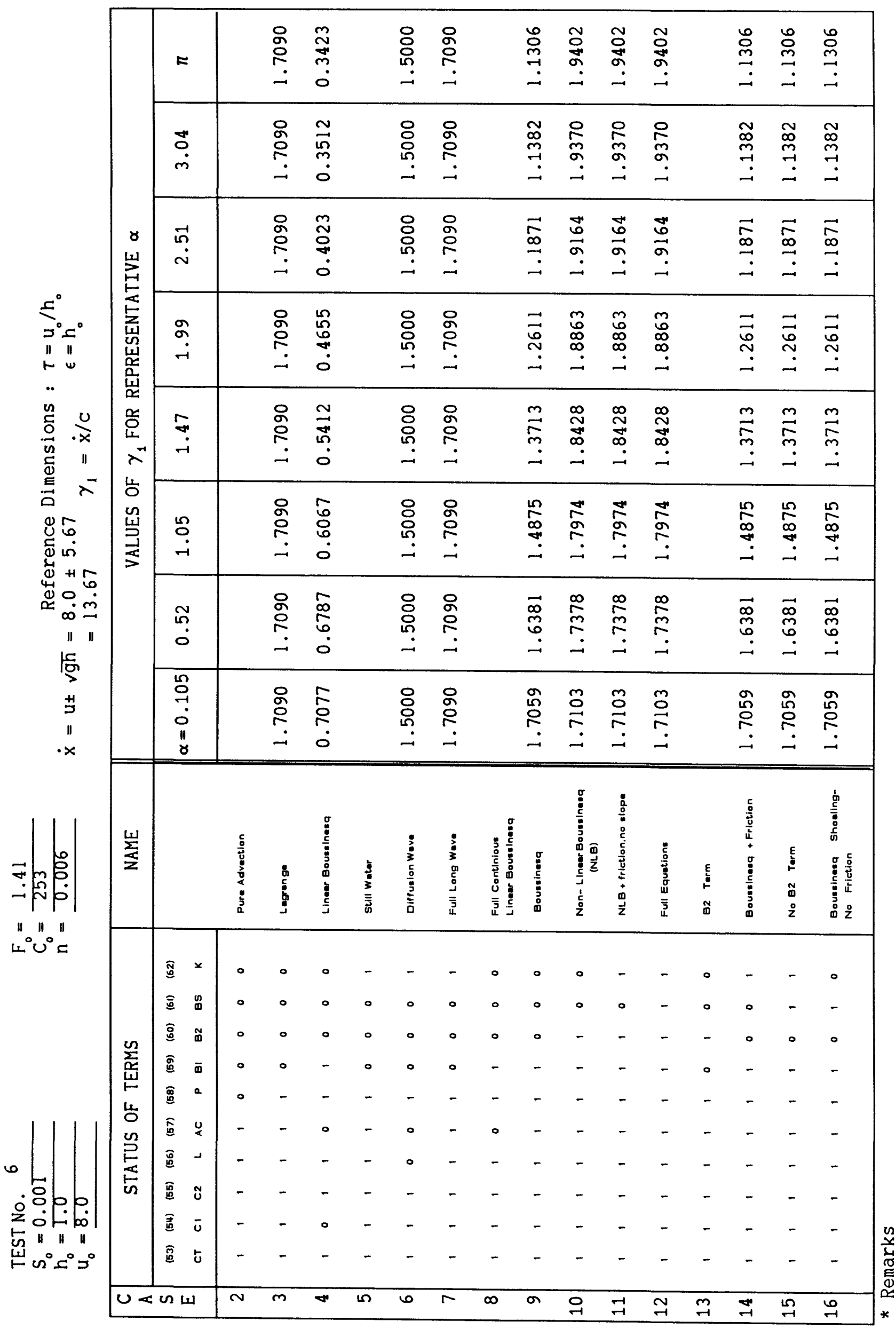




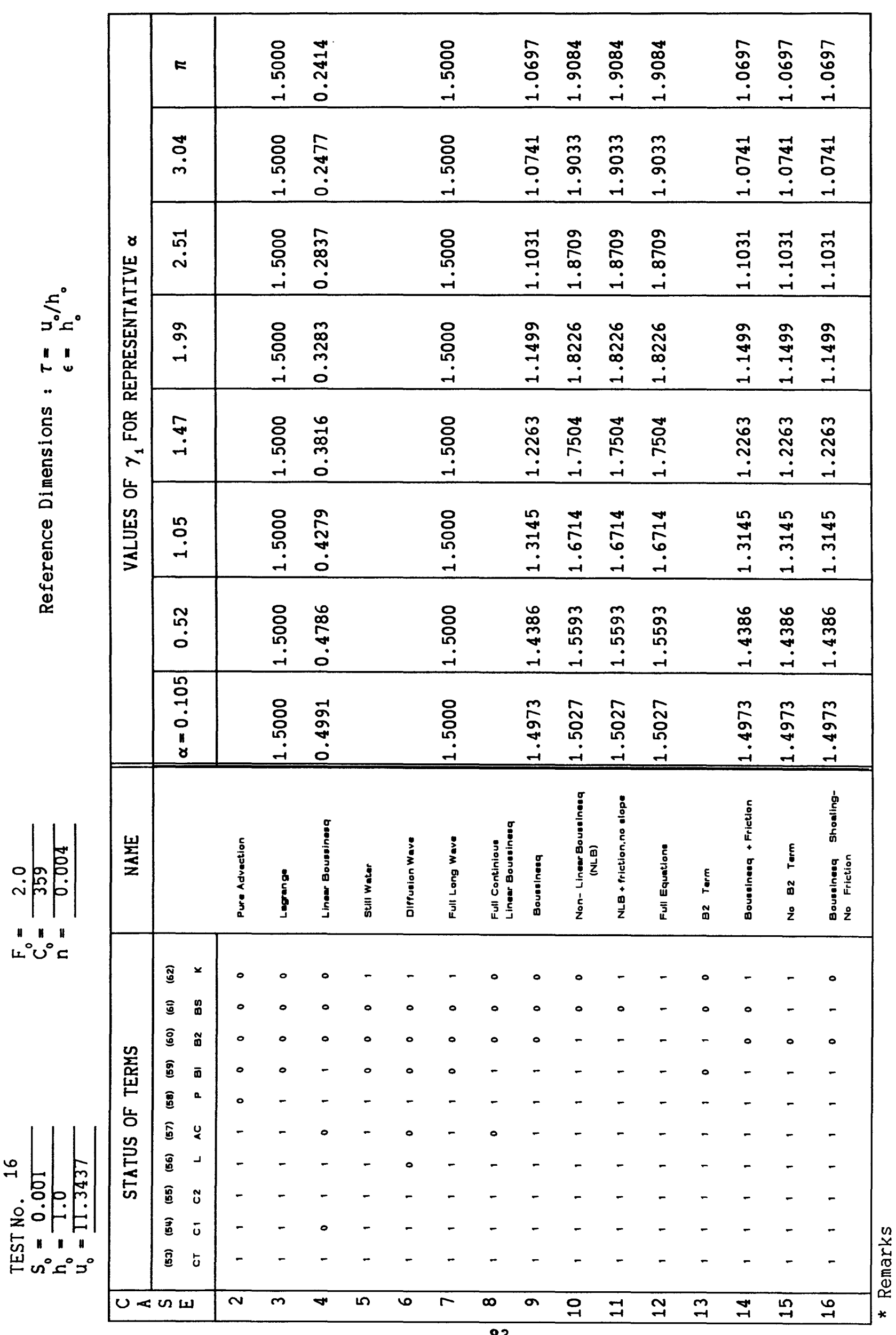




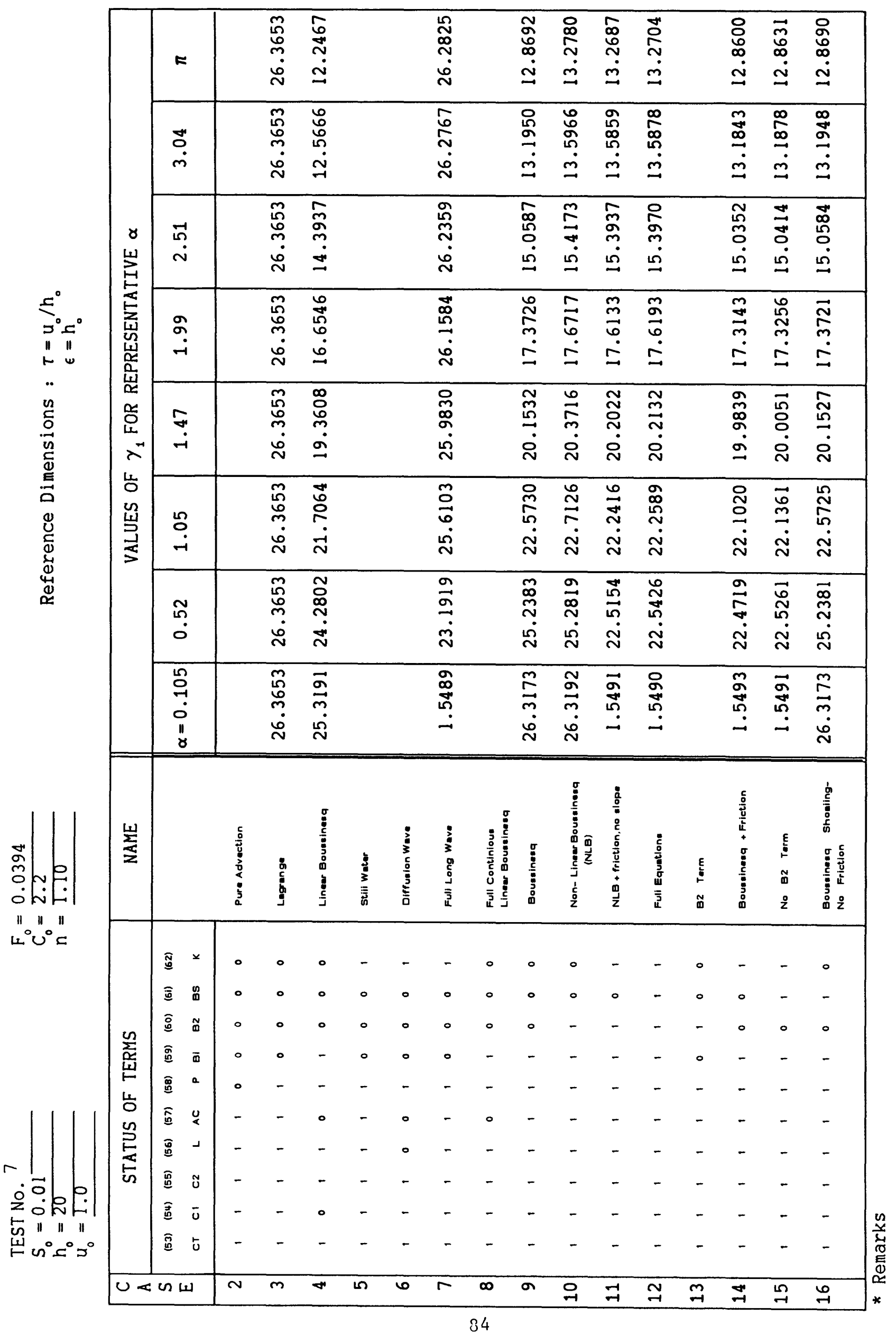




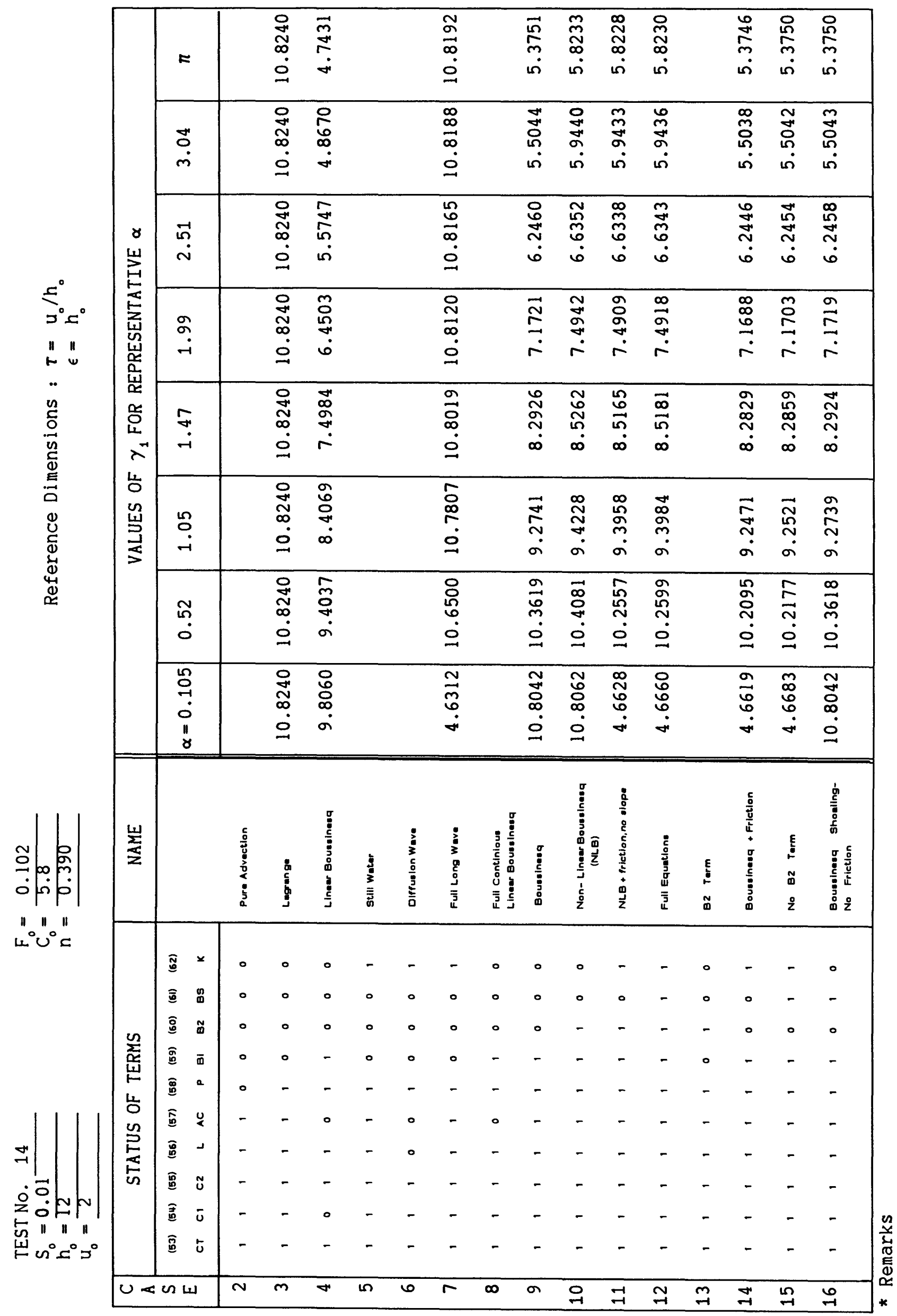




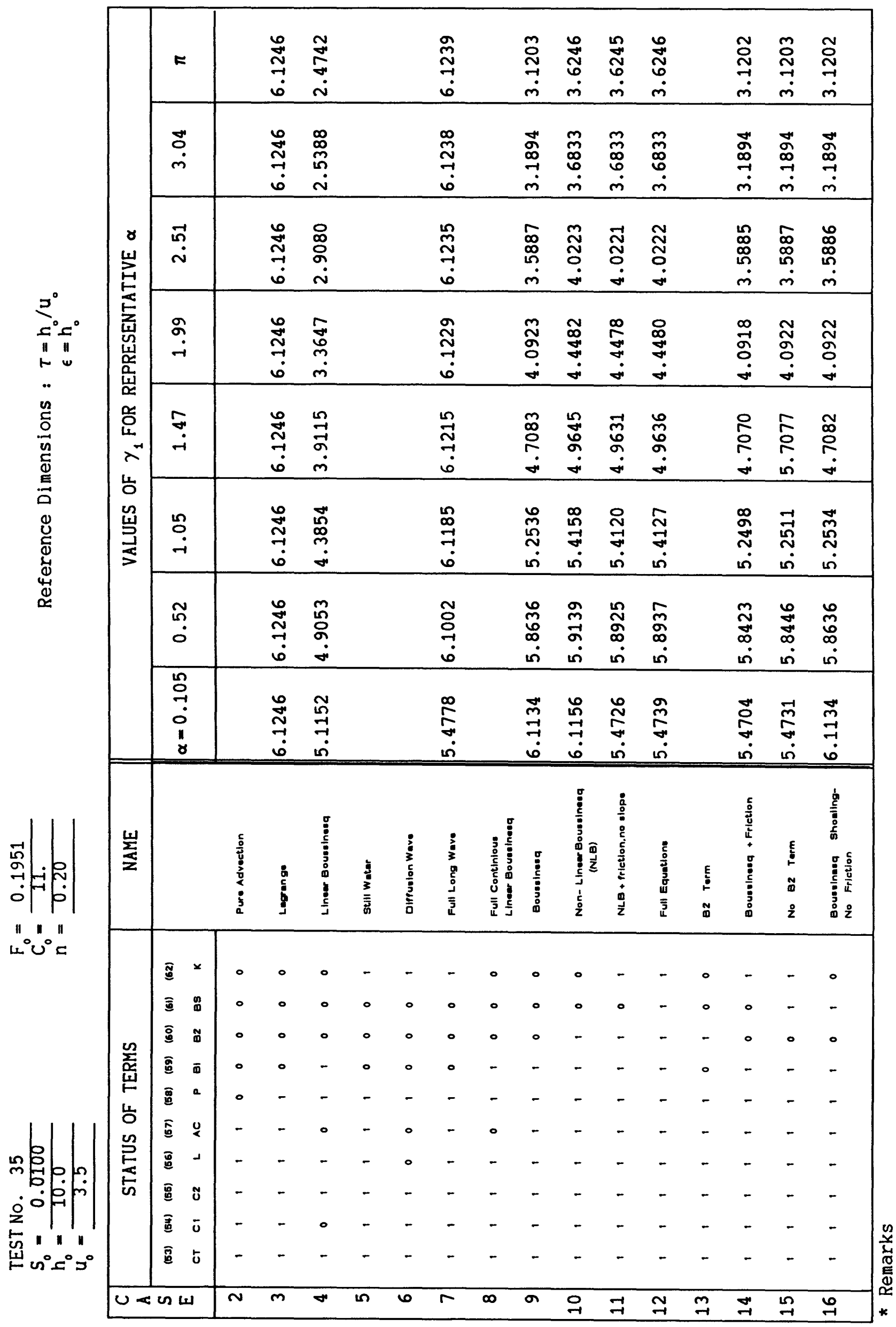




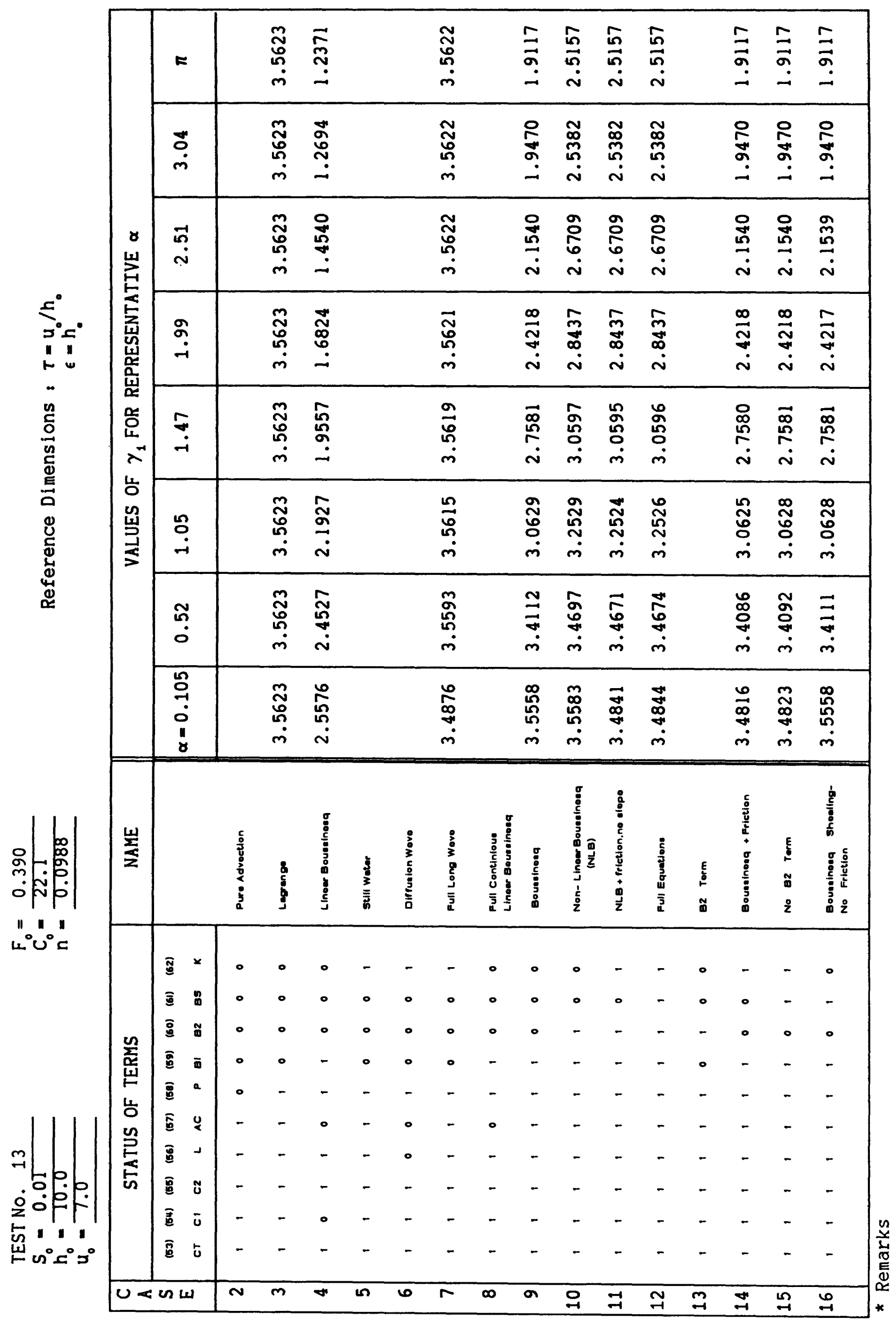




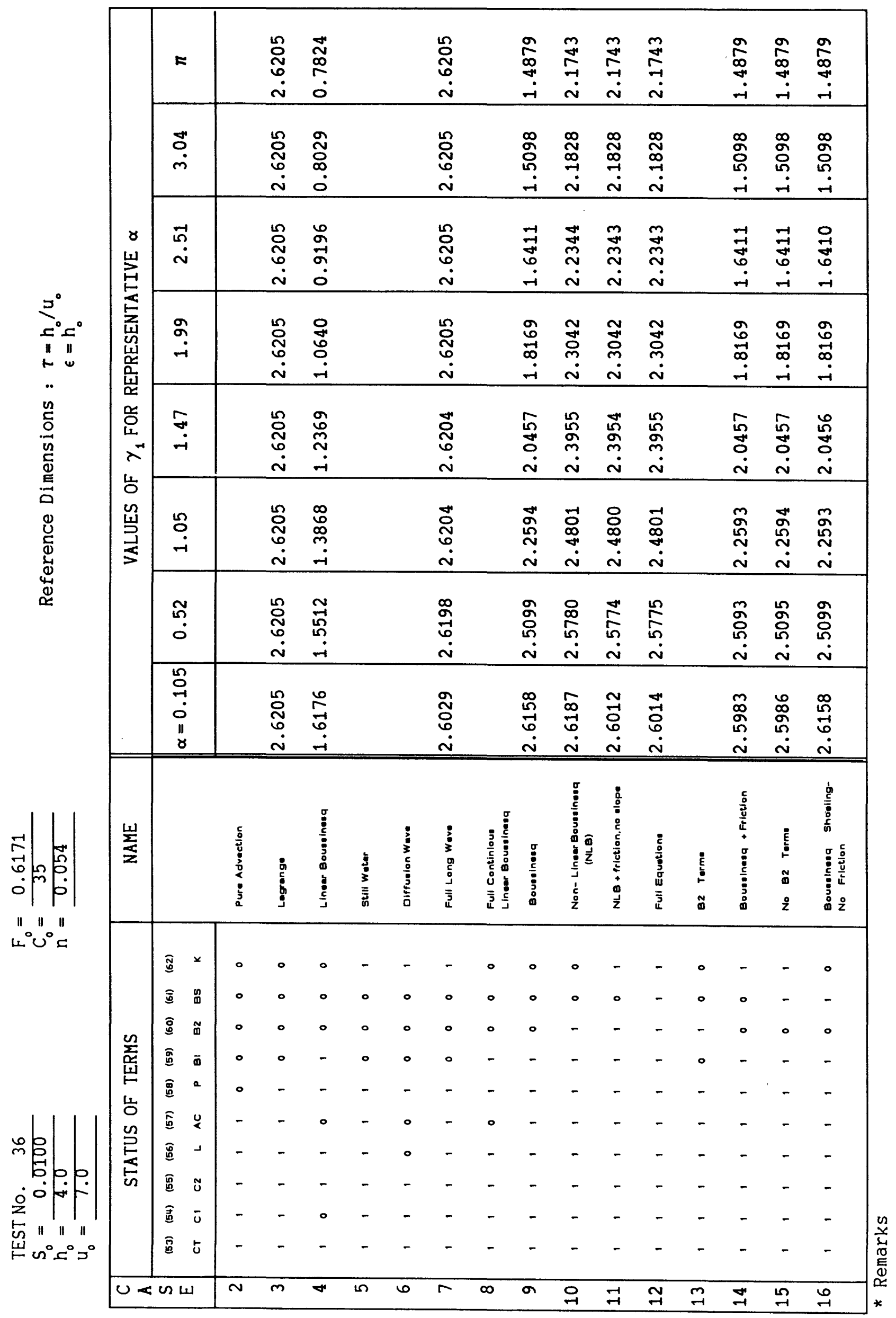




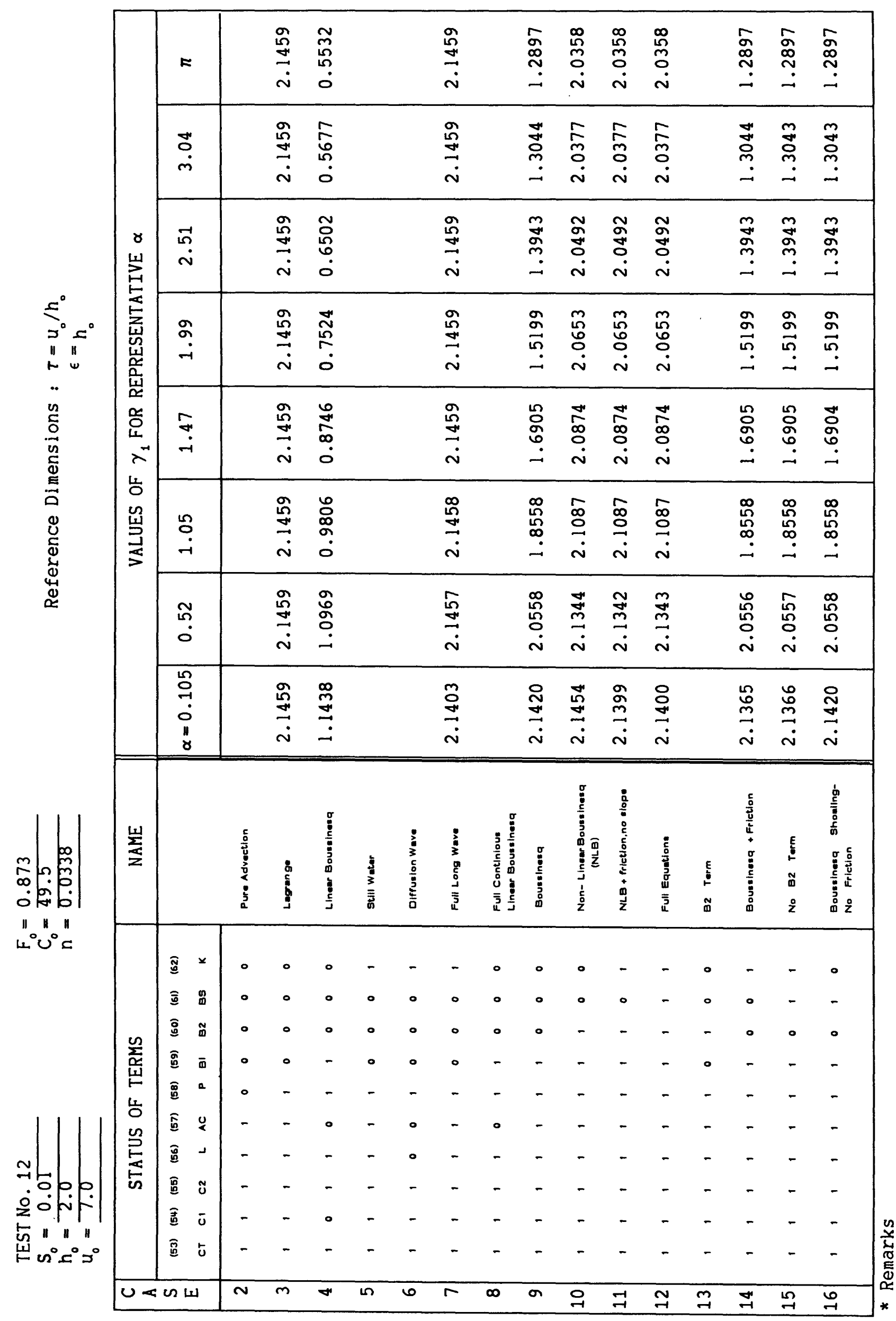




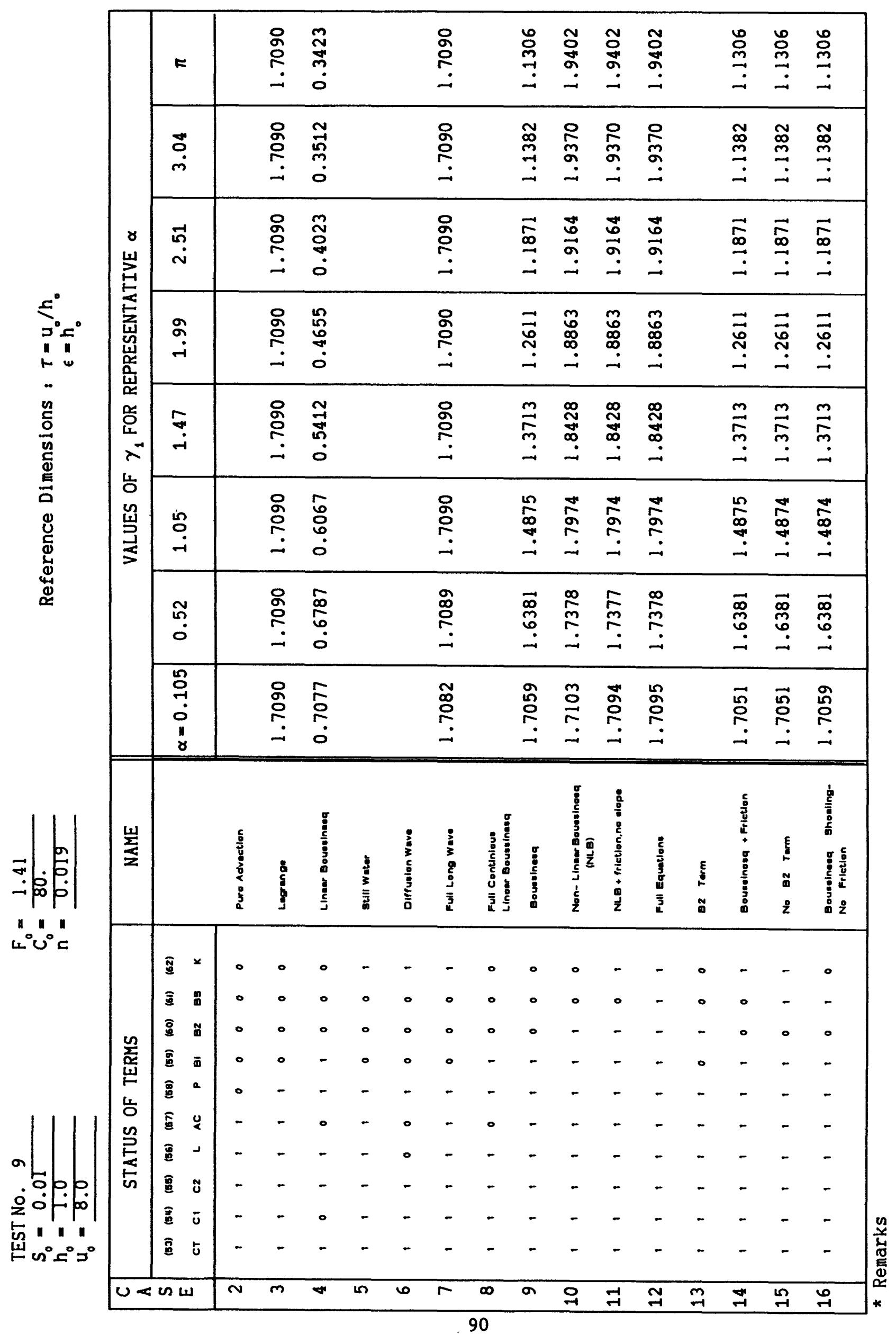




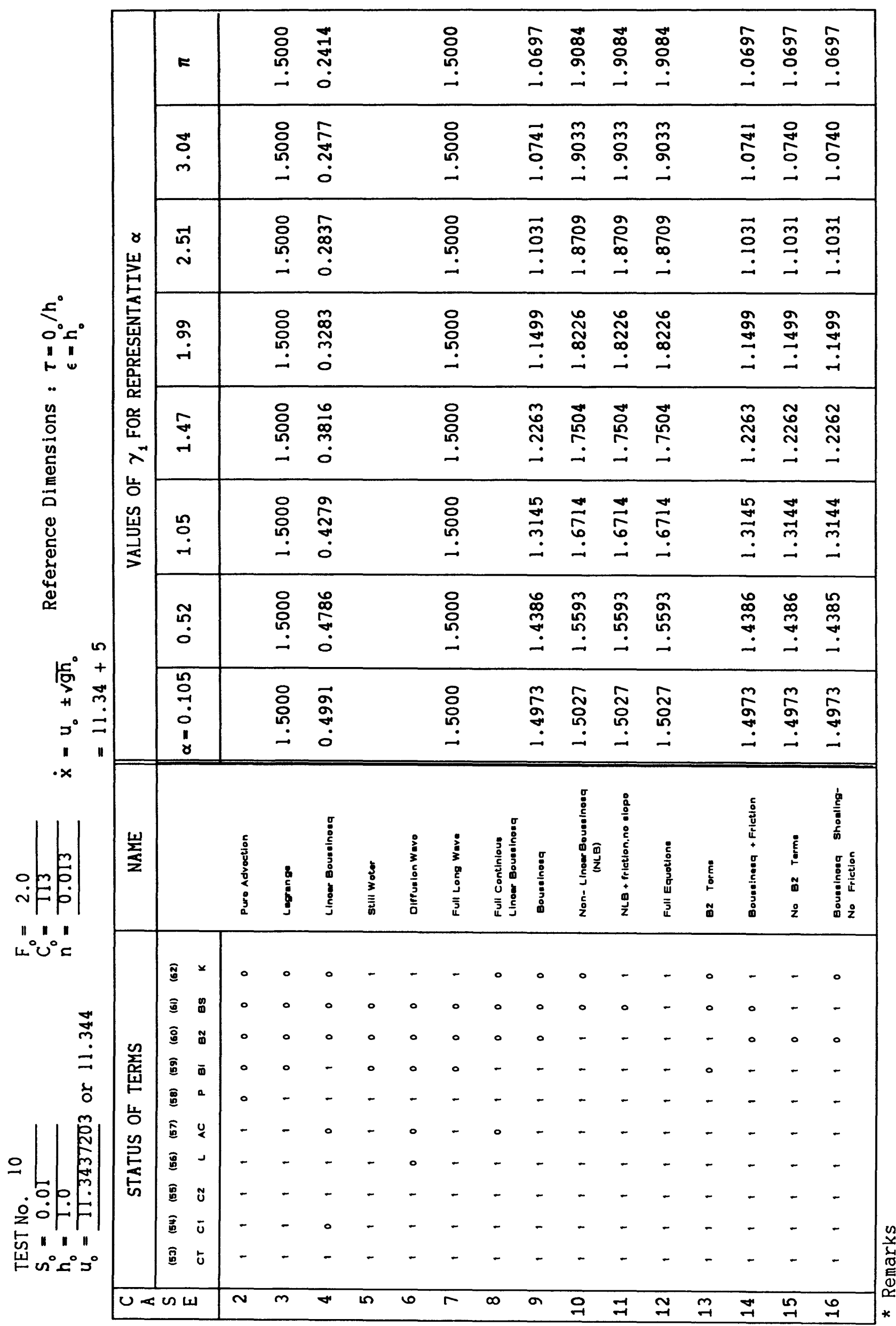




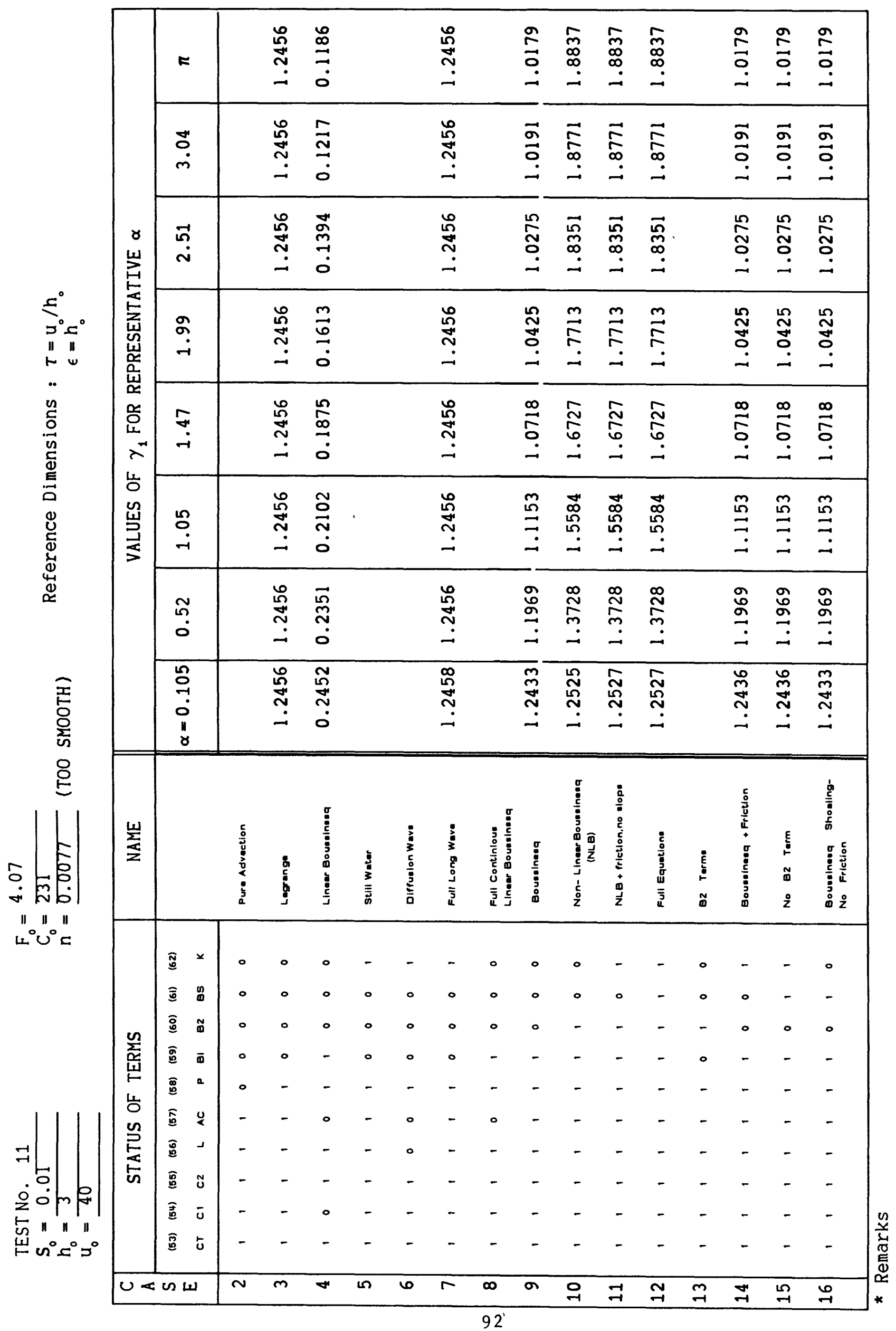




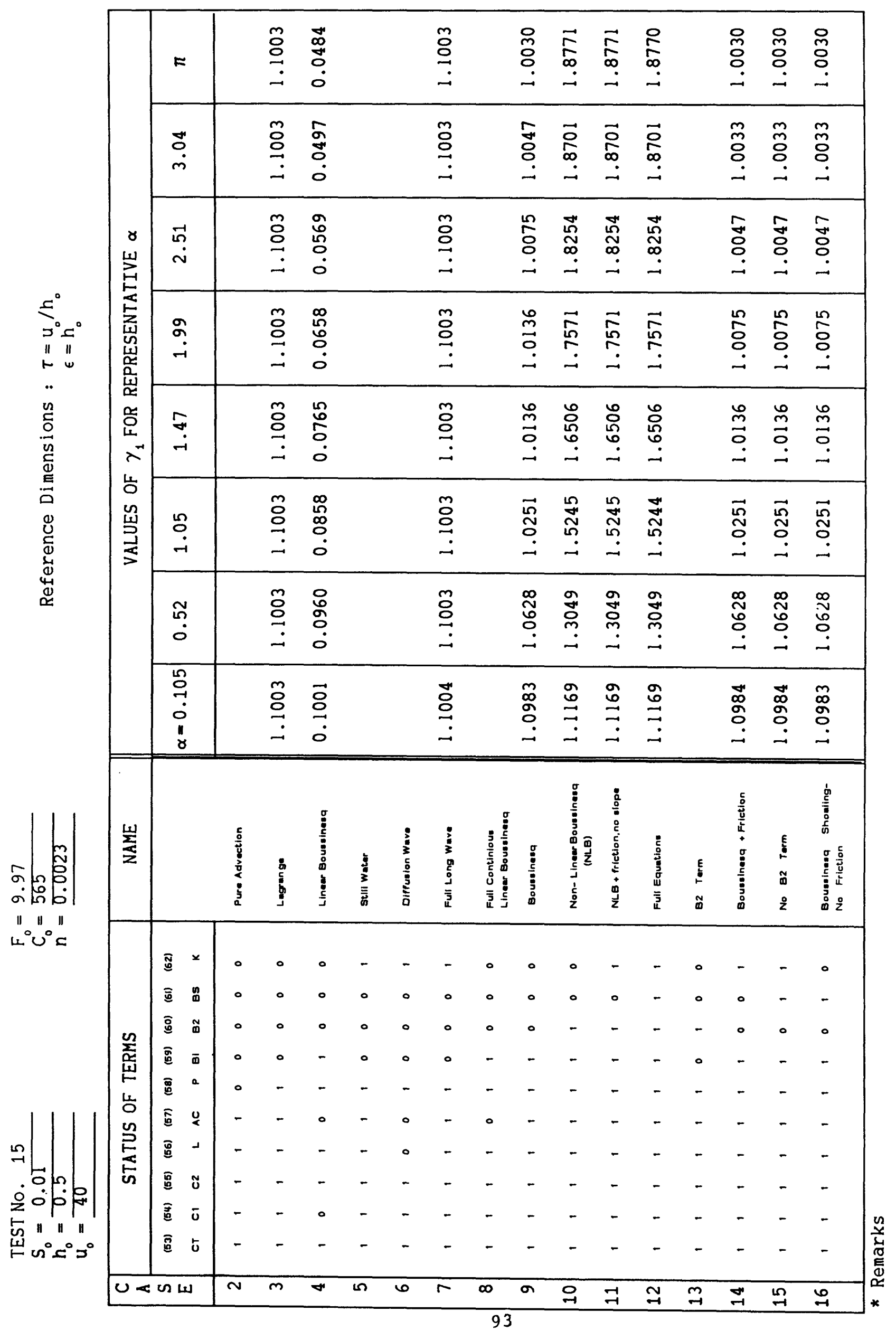




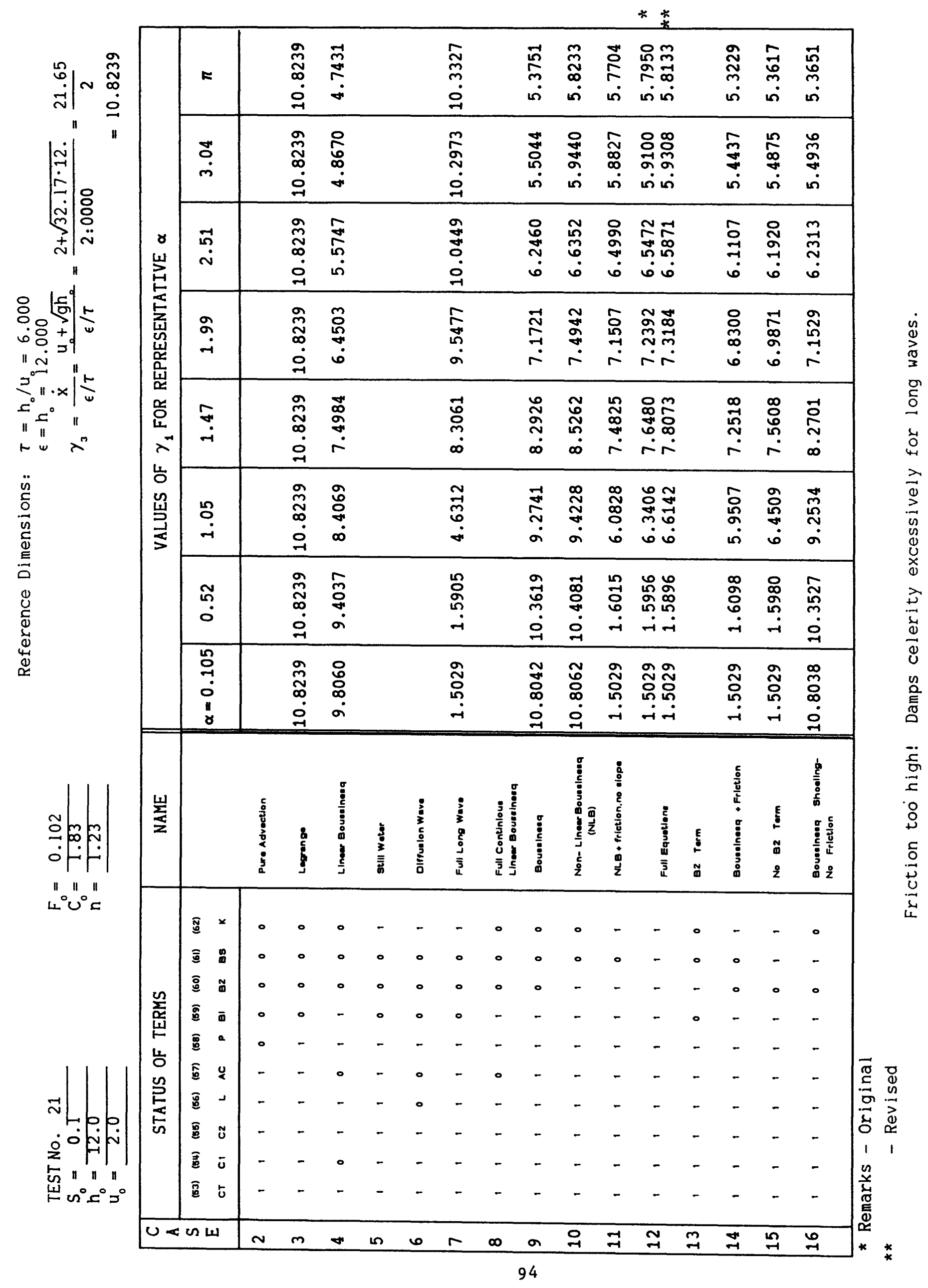




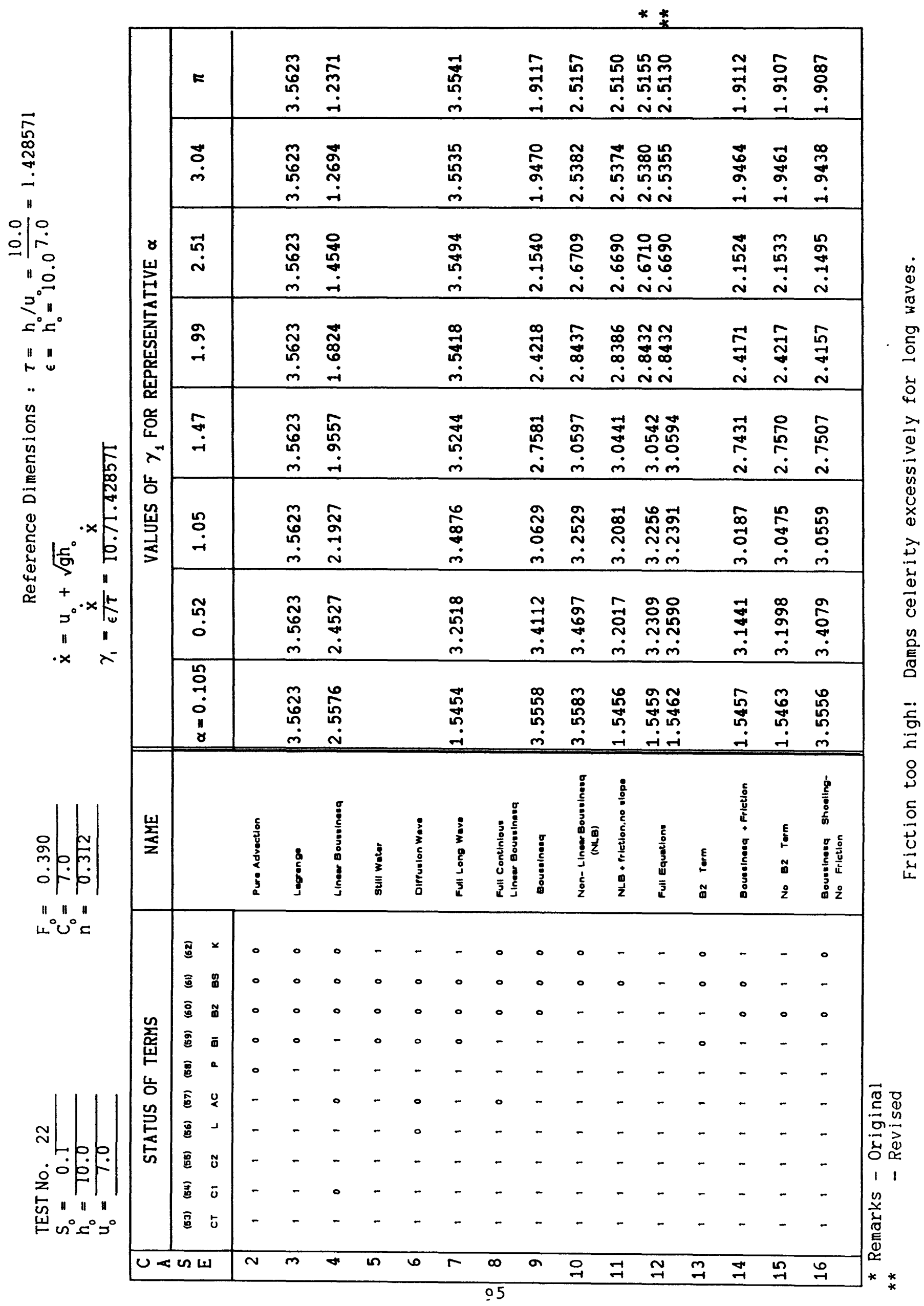




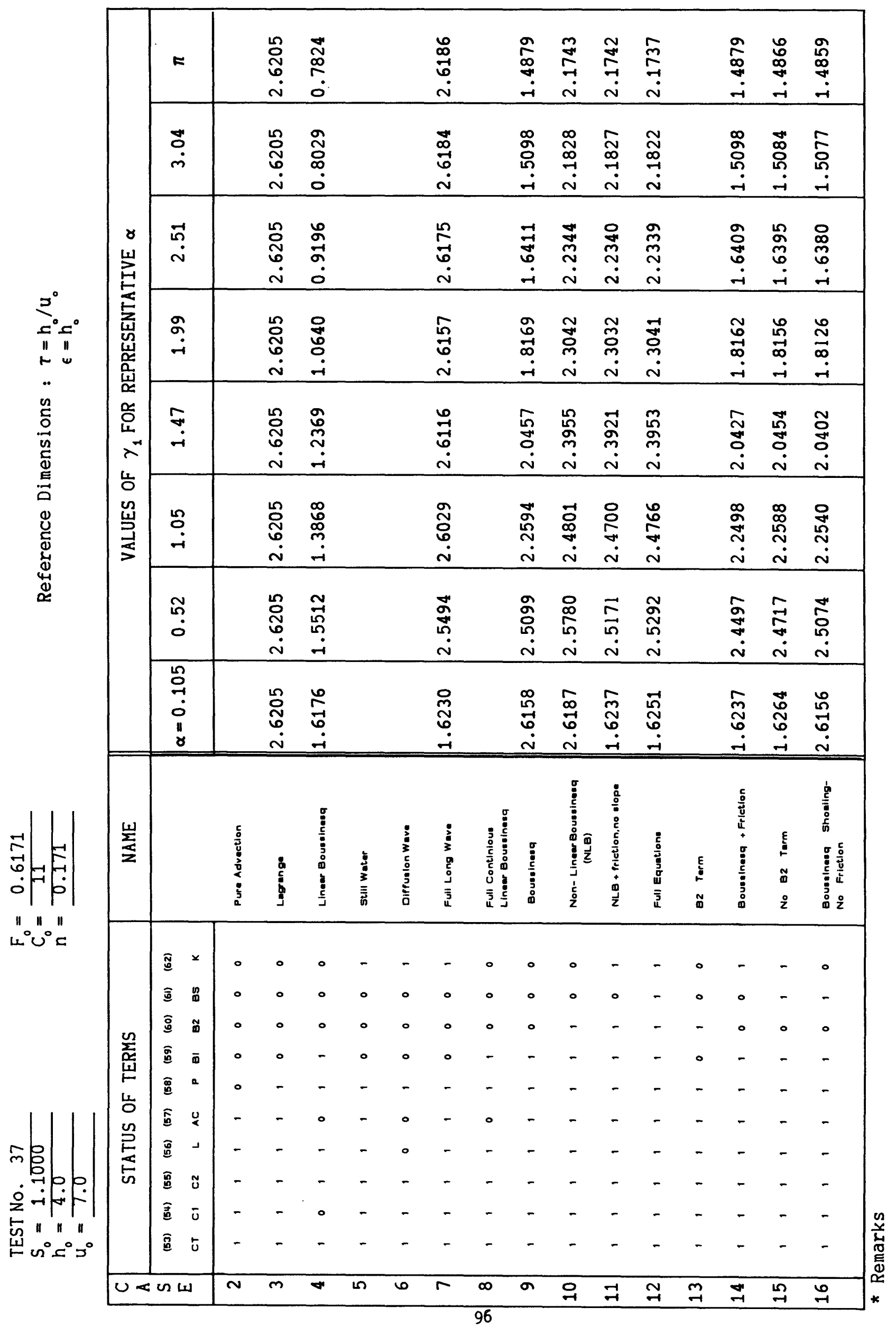




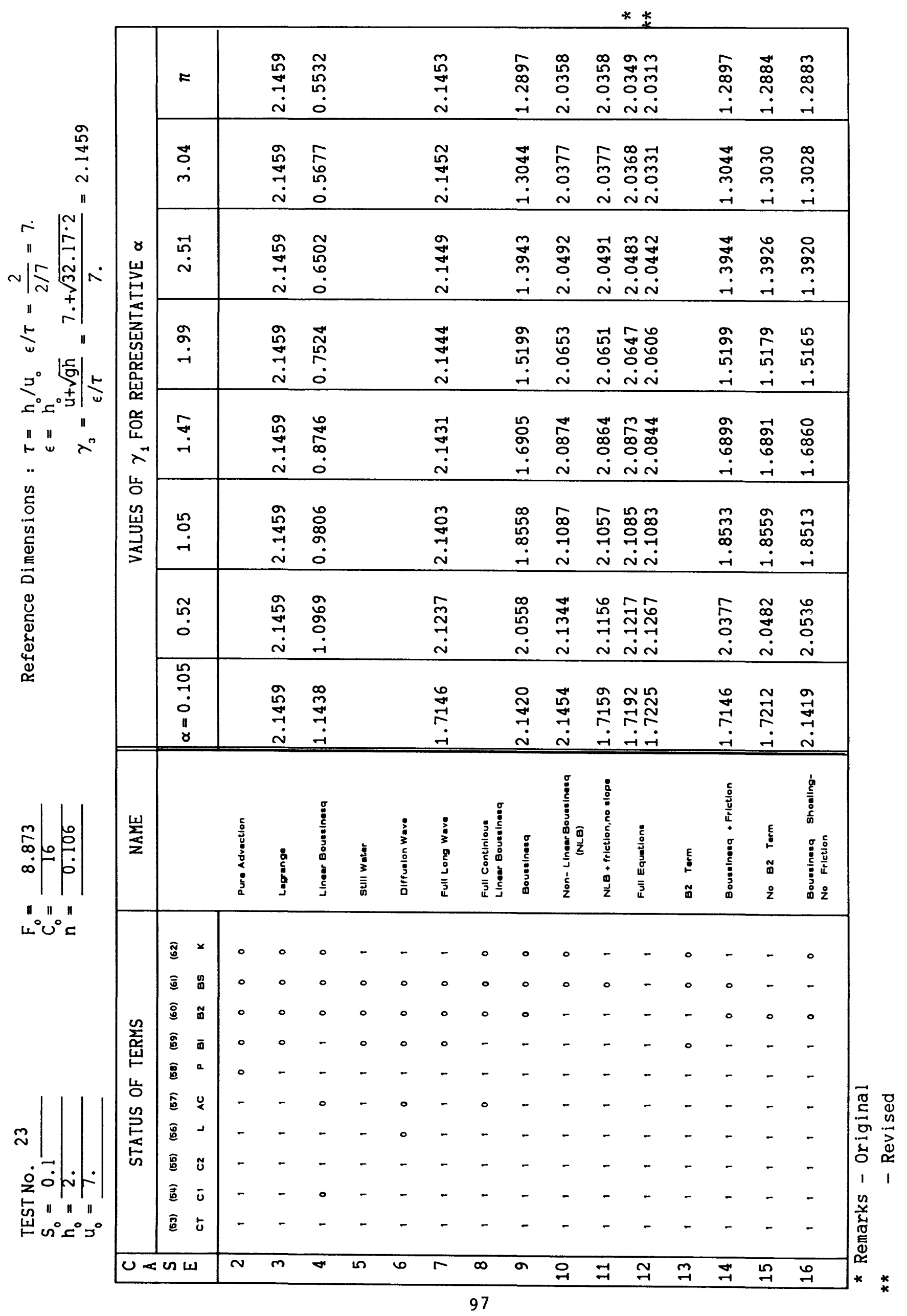


* $\underset{*}{*}$

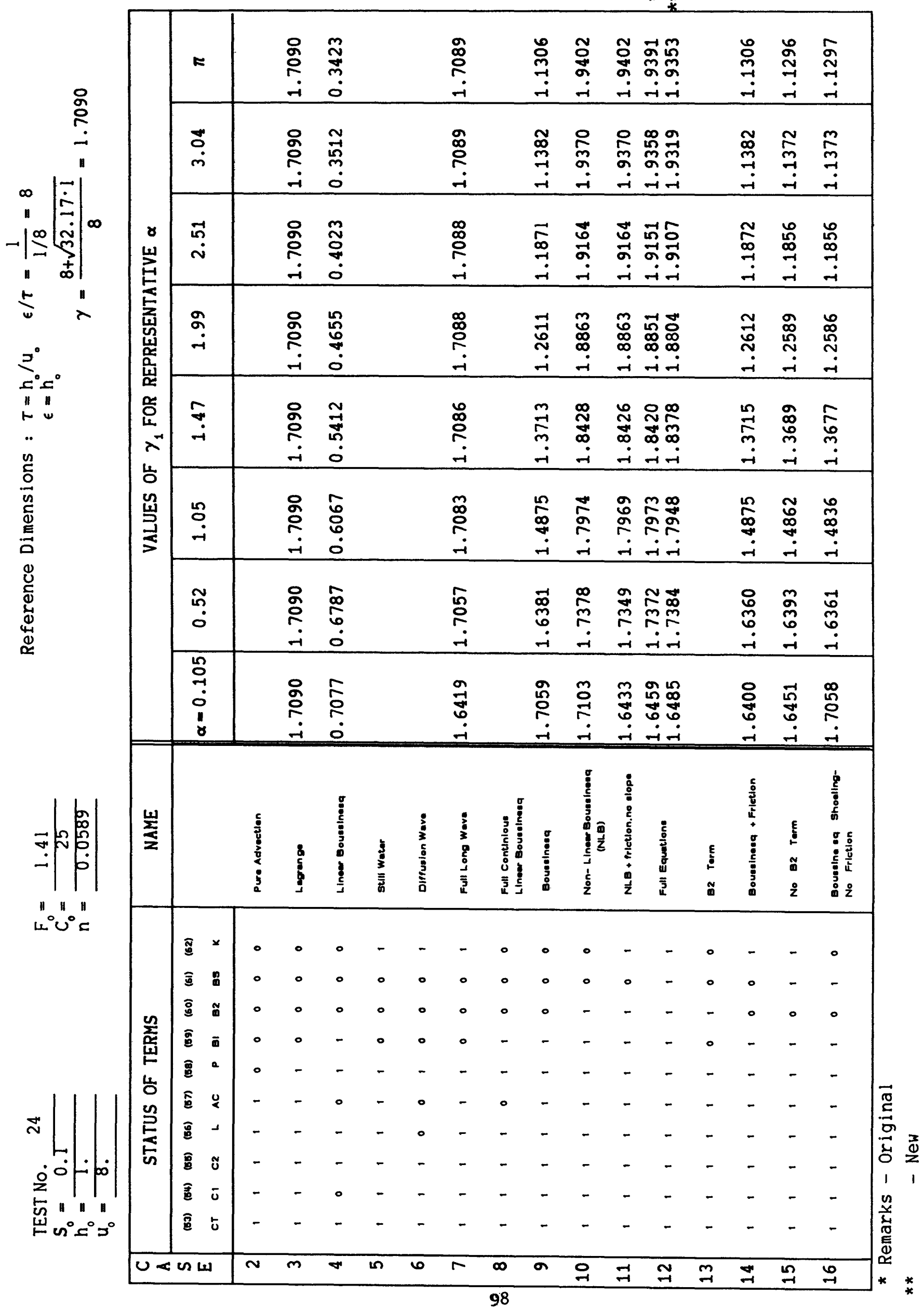




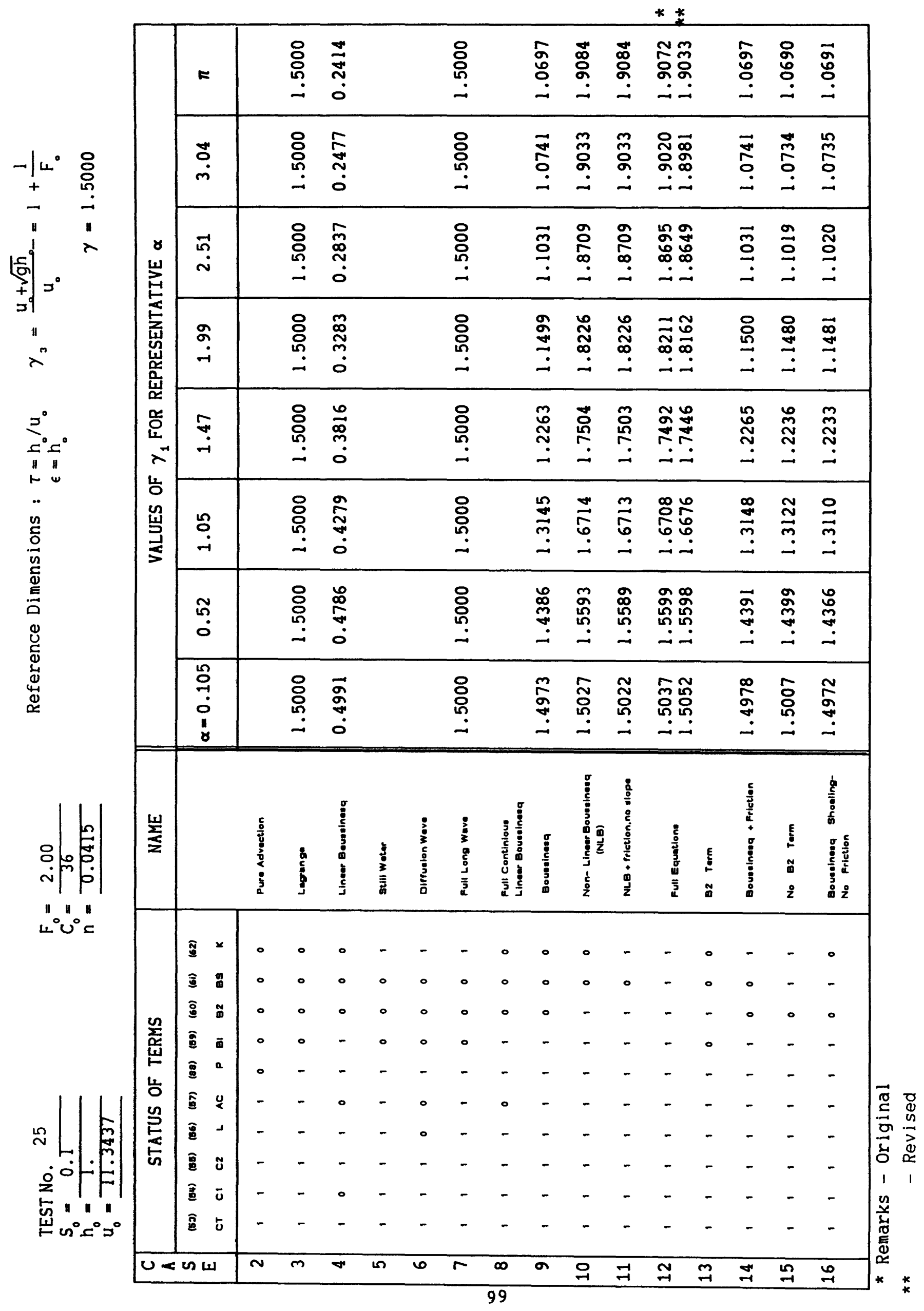




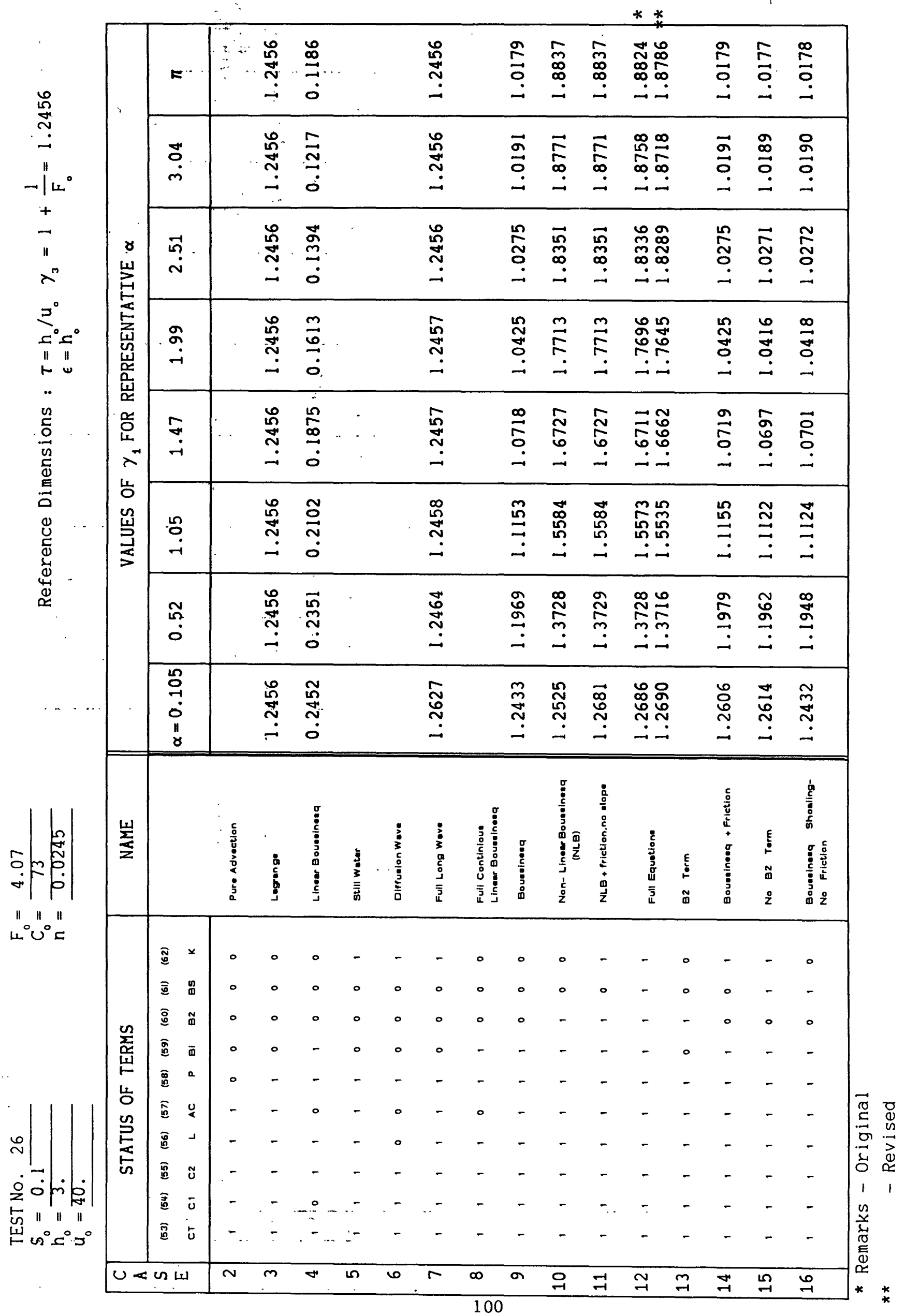




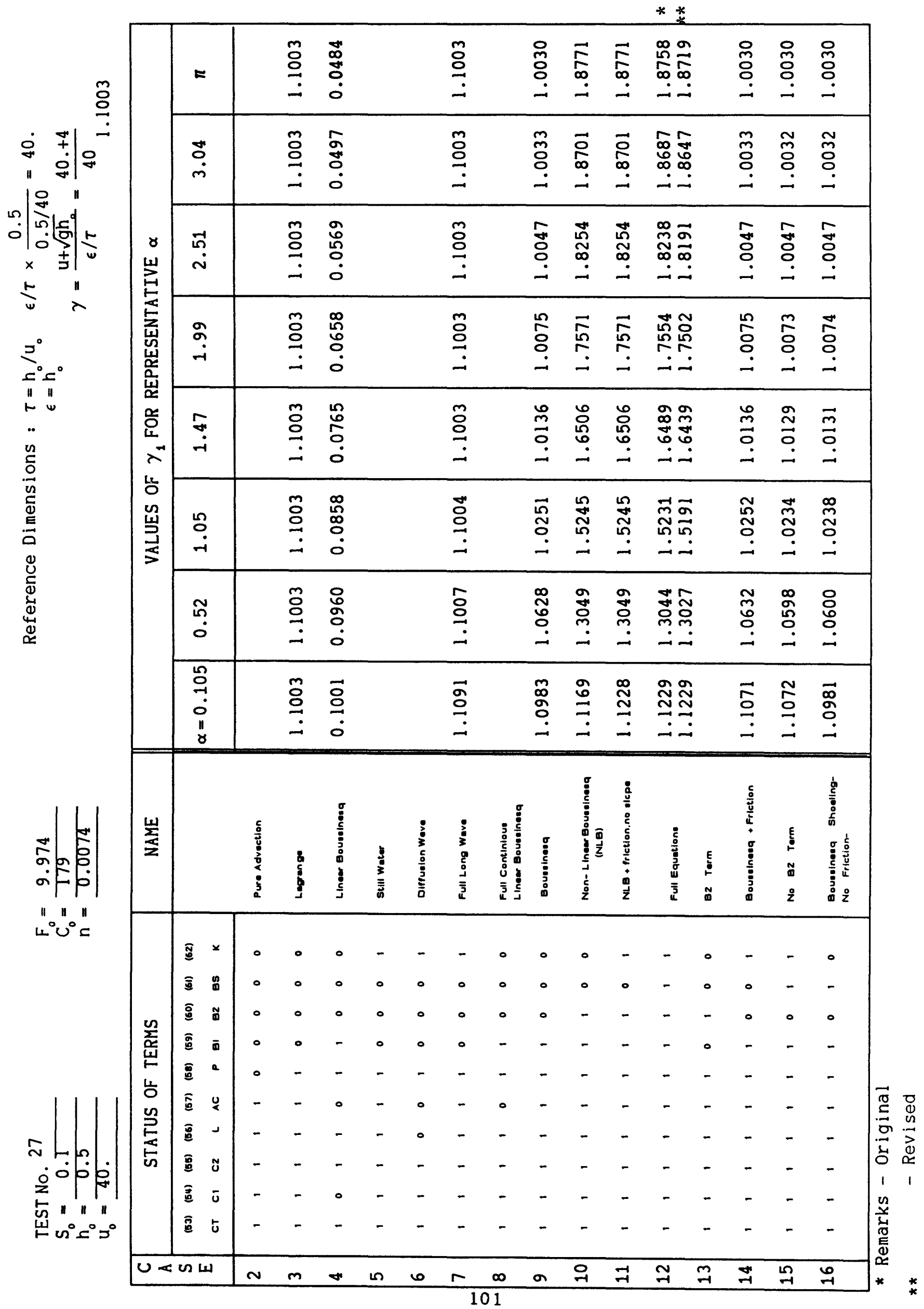

